\title{
Speedy Standing Wave Design of Size-Exclusion Simulated Moving Bed: Solvent Consumption and Sorbent Productivity Related to Material Properties and Design Parameters
}

George S. Weeden Jr. and Nien-Hwa Linda Wang*

School of Chemical Engineering, Purdue University, West Lafayette, IN 47907

*Email: wangn@ecn.purdue.edu, Phone: 765-494-4081, Fax: 765-494-0805 


\section{Abstract:}

Size-exclusion simulated moving beds (SEC-SMB) have been used for large-scale separations of linear alkanes from branched alkanes. While SEC-SMBs are orders of magnitude

4 more efficient than batch chromatography, they are not widely used. One key barrier is the

5 complexity in design and optimization. A four-zone SEC-SMB for a binary separation has seven

6 material properties and 14 design parameters (two yields, five operating parameters, and seven

7 equipment parameters). Previous optimization studies using numerical methods do not guarantee

8 global optima or explicitly express solvent consumption $(D / F)$ or sorbent productivity $\left(P_{R}\right)$ as

9 functions of the material properties and design parameters.

10 The Standing Wave concept is used to develop analytical expressions for $D / F$ and $P_{R}$ as

11 functions of 14 dimensionless groups, which consist of 21 material and design parameters. The

12 resulting Speedy Standing Wave Design (SSWD) solutions are simplified for two limiting cases:

13 diffusion or dispersion controlled. An example of SEC-SMB for insulin purification is used to

14 illustrate how $D / F$ and $P_{R}$ change with the dimensionless groups. The results show that

15 maximum $P_{R}$ for both diffusion and dispersion controlled systems is mainly determined by

16 yields, equipment parameters, material properties, and two key dimensionless groups: (1) the

17 ratio of step time to diffusion time and (2) the ratio of diffusion time to pressure-limited

18 convection time. A sharp trade off of $D / F$ and $P_{R}$ occurs when the yield is greater than $99 \%$. The

19 column configuration for maximum $P_{R}$ is analytically related to the diffusivity ratio and the

20 selectivity. To achieve maximum sorbent productivity, one should match step time, diffusion

21 time, and pressure-limited convection time for diffusion controlled systems. For dispersion

22 controlled systems, the axial dispersion time should be about 10 times the step time and about 50 
23 times the pressure-limited convection time. Its value can be estimated from given yields,

24 material properties, and column configuration. Among the material properties, selectivity and

25 particle size have the largest impact on $D / F$ and $P_{R}$. Particle size and 14 design parameters can

26 be optimized for minimum $D / F$, maximum $P_{R}$, or minimum cost on a laptop computer.

27 Keywords: Simulated moving bed; Size-exclusion; Standing Wave Design; Solvent

28 consumption; Sorbent productivity; Optimization 


\subsection{Introduction}

Size-exclusion chromatography (SEC) has many important applications. Examples

32 include gel permeation chromatography (GPC), for analysis of protein mixtures or obtaining

33 molecular weight distributions of polymers [1,2], and purification of proteins, such as human

34 insulin [3]. However, conventional SEC is a batch process and it is less efficient than simulated

35 moving bed (SMB) for large-scale production.

36 In SMB, the columns are connected in a circular configuration (loop). Inlet and outlet

37 ports divide the loop into different sections (zones) with different flowrates. A typical 4-zone

38 SMB with two columns per zone (2-2-2-2 configuration) is shown in Fig. 1. The ports are moved

39 periodically to follow the migrating solute bands. The time between port switches is called the

40 switching time, or step time $\left(t_{s}\right)$. The average port velocity $(v)$ is equal to the column length $\left(L_{c}\right)$

41 divided by the step time. The separation is achieved by containing the solutes in specific zones.

42 As seen in Fig. 1, the small green component (slow solute) is never present in Zone IV while the

43 large blue component (fast solute) is never present in Zone I. By containing the advancing and

44 trailing concentration waves in their respective zones, pure products can be continuously

45 removed.

46 Size-exclusion simulated moving beds (SEC-SMBs) are more efficient than conventional

47 SEC because only partial separation of solutes in the loop is required to obtain high-purity

48 products with high yield. As a result, a large fraction of the sorbent capacity is utilized and

49 product dilution is reduced. Thus, SMBs consume orders of magnitude less solvent, require an

50 order of magnitude less sorbent, and take up less space than batch operations. Because SMBs are 
51 continuous processes, they also require less manpower. These advantages make SMBs

52 economical for large-scale separations.

53 The SEC-SMB was first introduced by Universal Oil Products (UOP) in 1961 as the

54 Molex process, which separates linear alkanes from branched alkanes [4,5]. SMBs were later

55 developed for adsorptive systems, such as large-scale hydrocarbon purification and high fructose

56 corn syrup production [6]. SMBs for chiral separations have been developed since the 1990s [7].

57 Lab-scale SEC-SMB have been developed for insulin purification [8,9], separation of myoglobin

58 from bovine serum albumin (BSA) [10], lactose removal from human milk [11], and

59 polyethylene glycol (PEG) fractionation by molecular weight (MW) [12].

Even though SMBs have many advantages, they have not been widely used for large-

61 scale production. SMBs have complex transient and cyclic steady-state phenomena. Equipment

62 for SMBs is often more complex and expensive than batch equipment and SMB experiments are

63 costly and time-consuming. The most important barrier is the complexity of the design and

64 optimization of SEC-SMB. A four-zone SEC-SMB for a binary separation has 21 variables,

65 which include seven material properties and 14 design parameters, Fig. 2. The 14 design

66 parameters include two yield requirements $\left(Y_{i}\right)$, seven equipment parameters, and five operating

67 parameters. The seven material properties are bed void fraction $\left(\varepsilon_{b}\right)$, particle porosity $\left(\varepsilon_{p}\right)$, two

68 apparent retention factors $\left(\delta_{i}\right)$, two intraparticle diffusivities $\left(D_{p, i}\right)$, and particle size $\left(R_{p}\right)$. The two

69 yield requirements can also be specified as two purities or one yield and one purity. The seven

70 equipment parameters are column length $\left(L_{c}\right)$, dead volume $(D V)$, maximum pressure drop

$71\left(\Delta P_{\max }\right)$, and the column configuration (the number of columns in each zone, $\left.N^{j}\right)$. The five

72 operating parameters are the four zone velocities $\left(u_{0}^{j}\right)$ and port velocity $(v)$. Experimental trial 
73 and error with 14 design parameters would be extremely costly. Additionally, the seven material

74 properties, including particle size, can be optimized.

SEC-SMB systems can be optimized for maximum productivity, minimum solvent

76 consumption, or minimum cost. Cost optimizations need to incorporate three main costs:

77 equipment cost; solvent cost, which is related to solvent consumption; and sorbent cost, which is

78 related to sorbent productivity. These costs are controlled by the equipment, material properties, 79 and operating parameters.

80 The simplest method for designing the five operating parameters is the local equilibrium

81 theory or "triangle" theory. It is widely used and works well for ideal systems (no mass transfer

82 resistance) [13]. However, for non-ideal systems (with mass transfer resistance), this theory only

83 gives the range of possible operating parameters where separation of the components will occur.

84 It does not guarantee purity or yield and it does not give optimum operating parameters for non-

85 ideal systems (most low pressure systems).

86 The Standing Wave Design (SWD) was first developed by Ma and Wang in 1997 for

87 binary, linear adsorption systems with mass transfer resistances [14]. For fixed material

88 properties, yields, and equipment parameters, the SWD determines the five optimum operating

89 parameters to maximize productivity and minimize solvent consumption. It was extended to

90 multicomponent linear systems [15] and nonlinear systems [16-18]. Pressure limit

91 considerations were incorporated into the SWD [19] by checking that the resulting operating

92 parameters did not violate the pressure constraint.

93 The SWD method has been incorporated into various optimization routines, based on grid

94 search [9], genetic algorithms [20], simulated annealing [21,22], or combined simulated

95 annealing and genetic algorithm (SAGA) [23]. Optimization variables include particle size $\left(R_{p}\right)$, 
96 column length $\left(L_{c}\right)$, column configuration $\left(N^{j}\right)$, and yields $\left(Y_{i}\right)$ [24]. These techniques cannot

97 guarantee global optima and they do not provide an overview of how solvent consumption,

98 sorbent productivity, and separation cost are related to material properties and design parameters.

99 The objective of this work is to find analytical solutions for the solvent consumption and

100 productivity of SEC-SMB systems as functions of the equipment, material, and operating

101 parameters. These analytical solutions can then be used to understand how solvent consumption

102 and sorbent productivity are affected by the material and design parameters. These solutions can

103 also be used to quickly find the optimal designs for maximum productivity, minimum solvent

104 consumption, or lowest separation cost (with given cost functions). This new method is called

105 the Speedy Standing Wave Design (SSWD) method.

106 To produce the general analytical solutions, the SWD equations are solved in terms of

107 dimensionless groups. For a binary SEC-SMB separation, combining dimensionless groups with

108 the SWD equations reduces the total number of variables from 21 to 14 (Fig. 2). The details are

109 shown in Section 2. The general solutions are simplified for two limiting cases: diffusion or

110 dispersion controlled systems. The solvent consumption and sorbent productivity results from

111 this new method are compared to those from three SEC-SMB systems in the literature. The

112 effects of the dimensionless groups are explored for the diffusion or dispersion controlled cases

113 using an example from insulin purification.

114 Some highlights of this study are the following. Analytical solutions for solvent

115 consumption and productivity are found in terms of 14 dimensionless groups and they greatly

116 facilitate optimization of SEC-SMBs. With appropriate cost functions, the minimum cost SMB

117 design can quickly be determined. The column configuration for maximum productivity is

118 analytically related to the diffusivity ratio and the selectivity. There is a sharp trade-off for 
119 solvent consumption and sorbent productivity when yields are specified to be greater than $99 \%$.

120 For diffusion controlled systems, matching step time, diffusion time, and pressure-limited

121 convection time gives maximum sorbent productivity. Fifteen decision variables $\left(Y_{i}, R_{p}, L_{c}, D V\right.$,

$122 N^{j}, \Delta P, u_{0}^{j}$, and $v$ ) can be optimized on a laptop computer.

\section{$123 \quad 2.0$ Theory}

124 In this section, the basic concept of the Standing Wave Design (SWD) method reported

125 in the literature is briefly reviewed in Section 2.1. The development of new general equations

126 using dimensionless groups is explained in Section 2.2. These new equations are called the

127 Speedy Standing Wave Design (SSWD) because they provide an overview of the solvent

128 consumption, sorbent productivity, and cost over a wide range of design parameters. The SSWD

129 equations are simplified for diffusion- and dispersion-controlled systems in Sections 2.3 and 2.4,

130 respectively. Optimization of decision variables to achieve minimum solvent consumption,

131 maximum productivity, or minimum cost using the SSWD is discussed in Section 2.5.

\section{2.1 Standing Wave Design (SWD)}

133 The SWD for ideal and non-ideal, linear adsorption isotherm systems was first developed

134 by Ma and Wang in 1997. For an ideal system, the SWD matches the port velocity to the velocity 135 of the concentration wave of the "standing" component in each zone in a continuous moving bed.

136 For a non-ideal system, a difference in port velocity and wave velocities is used to confine

137 selected waves in their respective zones. This concept is illustrated for a binary, non-ideal system

138 in Fig. 3. The fast moving solute (insulin, component 1) is removed in the raffinate, while the

139 slow moving solute $\left(\mathrm{ZnCl}_{2}\right.$, component 2$)$ is removed in the extract. The arrows point to the 
140 wave which is confined in that zone. The equations for the design of linear systems with mass

141 transfer effects are presented in Eq. (1) [14],

$142 u O I=1+\phi \delta 2 v+\beta 2 I L I E b, 2 I+\phi \delta 2 v 2 K 2 I$

143 (1a)

$144 u O I I=1+\phi \delta 1 v+\beta 1 I I L I I E b, 1 I I+\phi \delta 1 v 2 K 1 I I$

145 (1b)

$146 u O I I I=1+\phi \delta 2 v-\beta 2 I I I L I I I E b, 2 I I I+\phi \delta 2 v 2 K 2 I I I$

$148 u O I V=1+\phi \delta 1 v-\beta 1 I V L I V E b, 1 I V+\phi \delta 1 v 2 K 1 I V$

149

$150 u F=u O I I I-u O I I$

151 where $u O j$ is the interstitial velocity of the fluid in zone $\mathrm{j}$ (zone velocity); $u F$ is the feed velocity;

$152 \phi$ is the phase ratio which is equal to $\left(1-\varepsilon_{b}\right) / \varepsilon_{b} ; \delta i$ is the apparent retention factor for component $\mathrm{i}$;

$153 v$ is the port velocity; $\beta i j$ is the natural logarithm of the ratio of the highest concentration to the

154 lowest concentration of the standing wave of component $\mathrm{i}$ in zone $\mathrm{j}$ and it is directly related to

155 the yield, Eq. (A1.1); $L j$ is the length of zone $\mathrm{j}$ and is equal to the product of $N^{j}$ and $L_{c} ; E b, i j$ is

156 the axial dispersion coefficient for component $\mathrm{i}$ in zone $\mathrm{j}$; and $\mathrm{Ki} \mathrm{j}$ is the lumped mass transfer

157 parameter for component $\mathrm{i}$ in zone $\mathrm{j}$. 
159 resistances in series for linear systems, as shown in Eq. (2).

$$
1 K i j=R p 215 K s e, i \varepsilon p D p, i+R p 3 k f, i
$$

162 where $K_{s e, i}$ is the size-exclusion factor for component i (fraction of the pore volume that can be

163 accessed by the component), $D_{p, i}$ is the pore diffusivity of component i, and $k_{f, i}$ is the film mass

164 transfer coefficient. For most low pressure systems, the film mass transfer resistance is negligible 165 compared to that of intraparticle diffusion or axial dispersion [25].

166 For linear systems, the apparent retention factor of component $\mathrm{i}$ is given by Eq. (3) [8]

$167 \delta i=\varepsilon p K s e, i+1-\varepsilon p K s e, i a i+D V 1-\varepsilon b$

168 where $a_{i}$ in Eq. (3) is the Langmuir "a" value for adsorption and $D V$ is the total dead volume as a

169 fraction of the total sorbent packing volume. For size-exclusion systems, there is no adsorption

170 so the equation for the retention factors is simplified into Eq. (4).

$171 \delta i=\varepsilon p K s e, i+D V 1-\varepsilon b$

172 Table 1 contains a summary of the definitions and descriptions of the major dimensionless

173 groups which will be derived in the rest of this section.

174 An often referred to parameter is the selectivity of a system. The definition of selectivity

175 for SEC- SMB is given in Eq. (5).

$176 \alpha=\delta 2 \delta 1$ 
178 the five operating parameters $\left(u O_{j}\right.$ and $\left.v\right)$. The maximum feed flow rate can be found by

179 increasing the set feed flow rate until the mass transfer limit does not allow further increase.

180 The SWD equations can be used to determine the five optimum operating parameters for

181 given yields, material properties, and equipment parameters.

\subsection{Speedy Standing Wave Design (SSWD)}

In this study, solvent consumption and sorbent productivity are expressed in terms of

184 dimensionless groups. Such solutions can be used to elucidate the effects of equipment, material,

185 and operating parameters on solvent consumption and sorbent productivity. Furthermore, designs

186 for minimum cost, maximum productivity, or minimum solvent consumption can be found very

187 quickly. This advanced SWD method is called the Speedy Standing Wave Design (SSWD)

188 method.

189 Eq. (1) is simplified using the dimensionless groups defined in Table 1. Two key

190 dimensionless groups are used to separate the mass transfer effects due to diffusion from the

191 mass transfer effects due to dispersion. The dimensionless group which is a ratio of diffusion rate

192 to convection rate is $N D, i$ and is defined in Eq. (6).

$N D, i=\phi \varepsilon p K s e, i D p, i R p 2 L c v=$ Diffusion rateConvection rate $=t s t D, i=$ Step

194 timeEffective diffusion time

195 The port velocity is chosen as the characteristic velocity. To separate the influence of the

196 column configuration from that of diffusion, column length, instead of zone length, is chosen as

197 the characteristic length. Particle radius $\left(R_{p}\right)$ is chosen as the characteristic diffusion length. A 
198 characteristic diffusion time $\left(t_{D, i}\right)$ can be defined as $R_{p}{ }^{2}$ divided by the effective diffusivity

$199(\phi \varepsilon p K s e, i D p, i)$, while a characteristic convection time can be defined as $L_{c}$ divided by $v$, or the

200 step time $\left(t_{s}\right)$ in SMB. Thus, $N_{D, i}$ can be thought of as the ratio of the step time to the diffusion

201 time. A large $N_{D, i}$ means that in the time between port switches, there is plenty of time for the

202 solute to diffuse through the sorbent particles, which in turn means that the wave spreading due

203 to diffusion is small. A small $N_{D, i}$ means that there is not enough time for the solute to diffuse

204 through the particle within the step time, which in turn means wave spreading due to diffusion is

205 significant.

206 For a binary separation, there are two $N_{D, i}$ values, one for each component. These two

$207 N_{D, i}$ are related by the definition of the dimensionless group as shown in Eq. (7)

$208 \quad N D, 2 N D, 1=K s e, 2 D p, 2 K s e, 1 D p, 1=\lambda \gamma$

210 where $\lambda$ is the ratio of the size-exclusion factors and $\gamma$ is the ratio of the intraparticle diffusivities.

211 The dimensionless group which is a ratio of convection rate to axial dispersion rate is the

212 Peclet number, and is defined in Eq. (8).

$213 P e b i j=v L c E b, i j=$ Convection rateDispersion rate $=t$ Daxt $s=$ Axial

214 dispersion timeStep time

215 The characteristic dispersion time $\left(t_{D a x}\right)$ can be defined as $L_{c}{ }^{2}$ divided by the dispersion

216 coefficient $\left(E_{b, i}^{j}\right)$, while a characteristic convection time can be defined as $L_{c}$ divided by $v$, or the

217 step time $\left(t_{s}\right)$. The Peclet number can also be thought of as the ratio of a characteristic time for

218 dispersion $\left(t_{D a x}\right)$ to the step time $\left(t_{s}\right)$. If the Peclet number is very large, then the step time is

219 much smaller than the dispersion time and thus there is a very small effect of dispersion on the 
220 wave spreading. If the Peclet number is small, then the dispersion time is closer to the step time 221 and the effects from dispersion are significant.

222 For a four-zone SMB, there are four Peclet numbers, but they are related by Eq. (9)

$223 \quad P e b I V P e b j=E b j E b I V=\Gamma j$

224

225 where $\Gamma^{j}$ is the ratio of the axial dispersion coefficient of zone $\mathrm{j}$ to the axial dispersion 226 coefficient of Zone IV.

227 Eqs. (6-9) allow for Eq. (1) to be written using the $N_{D, i}$ of one component and the $P_{e b}{ }^{j}$ of 228 one zone and are shown in Eq. (10).

$229 u O I=v 1+\phi \delta 2+\beta 2 I \Gamma I P e b I V N I+\phi 2 \beta 2 I \delta 2215 N D, 1 \lambda \gamma N I$

$230 u O I I=v 1+\phi \delta 1+\beta 1 I I \Gamma I I P e b I V N I I+\phi 2 \beta 1 I I \delta 1215 N D, 1 N I I$

$231 u O I I I=v 1+\phi \delta 2-\beta 2 I I I I I I I P e b I V N I I I-\phi 2 \beta 2 I I I \delta 2215 N D, 1 \lambda \gamma N I I I$

$232 u O I V=v 1+\phi \delta 1-\beta 1 I V P e b I V N I V-\phi 2 \beta 1 I V \delta 1215 N D, 1 N I V$

233 For large-scale production, the solvent consumption of the SMB is an important factor for

234 the separation cost. Solvent cost is related to a ratio of the desorbent flow rate $(D)$ and the feed

235 flow rate $(F)$. This ratio $(D / F)$ is also related to the dilution of the products. $D$ and $F$ are

236 determined by Eq. (11) and Eq. (12), respectively where $S$ is the cross-sectional area of a

237 column.

$238 D=\varepsilon b S(u O I-u O I V)$

$239 F=\varepsilon b S(u O I I I-u O I I)$ 
240 Taking the ratio of Eq. (11) to Eq. (12) and substituting the zone velocities with Eq. (10) results

241 in Eq. (13).

$242 D F=\phi \delta 2-\delta 1+1 P e b I V \beta 2 I \Gamma I N I+\beta 1 I V N I V+\phi 2 \delta 1215 N D, 1 \beta 2 I \alpha 2 \lambda \gamma N I$

$243+\beta 1 I V N I V \phi \delta 2-\delta 1-1$ PebIV $\beta 2 I I I \Gamma I I I N I I I+\beta 1 I I \Gamma I I N I I-$

$244 \phi 2 \delta 1215 N D, 1 \beta 2 I I I \alpha 2 \lambda \gamma N I I I+\beta 1 I I N I I$

245 The $v, \varepsilon_{b}$, and $S$ all cancelled out in Eq. (13). This solution applies to systems where both

246 diffusion and dispersion are significant. For an ideal SMB with no mass transfer spreading, the

247 terms with $N_{D, l}$ or $P e b I V$ are negligible and the value of $D / F$ is 1.

248 Another useful parameter for evaluating SMB designs is productivity. Productivity $\left(P_{R}\right)$ 249 has dimensions (usually in mass of product per mass of sorbent per time). Eq. (14) defines $P_{R}$ in 250 the way that it will be discussed throughout this work.

$251 P R, i=\varepsilon b S u O I I I-u O I I C F, i Y i S L c N(1-\varepsilon b) \rho p$

$252 C_{F, i}$ is the feed concentration of component i (mass solute / volume of feed), $N$ is the total

253 number of columns in the SMB, and $\rho_{p}$ is the particle density (mass sorbent / particle volume).

254 Substituting the zone velocities in Eq. (14) with Eq. (10) results in Eq. (15).

$255 P R, i=Y i C F, i N t s \phi \rho p \phi \delta 2-\delta 1-1$ PebIV $\beta 2 I I I \Gamma I I I N I I I+\beta 1 I I \Gamma I I N I I-\phi 2 \delta 12$

$25615 N D, 1 \beta 2 I I I \alpha 2 \lambda \gamma N I I I+\beta 1 I I N I I$

257 For an ideal system, the terms with $N_{D, 1}$ and $P_{e b}{ }^{I V}$ are negligible and the productivity is the first 258 term on the right-hand side of Eq. (15). 
260 (17), respectively.

$261 N D *=\delta 2-\delta 1 N D, 1 \phi \delta 12=N D, 2 \delta 2-\delta 1 \lambda \gamma \phi \delta 12$

$263 P e b \star=\phi \delta 2-\delta 1 P e b I V=\phi \delta 2-\delta 1 P e b j \Gamma j$

264 After substituting Eqs. (16-17) into Eqs. (10,13), the resulting $u O_{j}$ equations are presented in the

265 first column of Table 2 and are given by Eq. (18), Table 2. The $D / F$ and $P_{R}$ expressions resulting 266 from using Eq. (18) for the zone velocities are shown in Table 2, Eqs. (19) and (20), respectively.

267 The column configuration which achieves the highest $P_{R}$ can be obtained from Eq. (20).

268 For a fixed total number of columns $(N)$, the question is how to distribute the columns between 269 the zones. Since $N^{I}$ and $N^{I V}$ do not appear in Eq. (20), columns placed in these zones do not 270 affect $P_{R}$ with the same operating conditions. This means Zones I and IV should have the 271 minimum number of columns (i.e. one). Increasing $N^{I I}$ or $N^{I I I}$ will increase $P_{R}$. The column

272 configuration that yields the maximum productivity can be obtained by taking the partial 273 derivative of Eq. (20) with respect to $N^{I I}$, or $N^{I I I}$, and setting the resulting equation equal to zero.

274 The fractional column configuration for maximum $P_{R}$ is given by Eq. (21).

$275 \quad N I N=1 N$

$276 N I I N=N I I I N \beta 1 I I \Gamma I I P e b *+115 N D * \beta 2 I I I I I I I P e b *+\alpha 215 N D * \lambda \gamma$ 
280 For systems where diffusion and dispersion are significant, the column configuration that results

281 in the highest $P_{R}$ is dependent on both $N_{D}{ }^{*}$ and $P_{e b}{ }^{*}$. Once these two dimensionless groups, the

282 material properties, yields, and the total number of columns are fixed, the optimum configuration 283 can easily be calculated.

Previous studies have shown that the true moving bed assumption holds for SMBs with

285 two or more columns per zone [14]. Having only one column in a zone may violate the true

286 moving bed assumption of the SWD. If this assumption is violated, the purities and yields can be

287 lower than those specified by SWD. Column configurations that have a zone with one column

288 should be simulated to ensure that the waves are actually confined [9]. To avoid the need for

289 simulations, the minimum number of columns per zone is set to be two in this study.

290 A major factor for equipment cost and sorbent cost is a pressure limitation. Systems may

291 be limited by pressure if the sorbent is very soft. In this case, the pressure drop per packing

292 length is limited [9]. Other systems may be limited by the maximum pressure allowed by pumps,

293 valves, or columns. The pressure drop across a uniformly packed bed of monodisperse, spherical

294 particles can be estimated using the simplified Ergun equation [26].

$295 \Delta P=37.5 \mu L c u O I \phi 2 R p 2$

$296 \Delta P$ is the pressure drop and $\mu$ is the viscosity of the fluid. Because the velocity in Zone I is

297 always the largest in SEC-SMB, it is used to calculate the maximum pressure drop $\left(\Delta P_{\max }\right)$

298 across a column in the SMB. Substitution of Eq. (18a) into Eq. (22) and rearranging results in 299 Eq. (23). 
$300 \Delta P$

$301 \quad R p 237.5 \phi \mu L c \nu=\phi 1+\phi \delta 2+\beta 2 I \Gamma I \phi \delta 2-\delta 1 P e b * N I+\phi \beta 2 I \alpha 2 \delta 2-\delta 115 N D * \lambda \gamma$

$302 \quad N I=N \Delta \mathrm{P}=t s t c I$

303 This dimensionless group, $N_{A}$, can be considered as a dimensionless pressure drop, which is

304 equal to the ratio of step time to the pressure-limited convection time through a column in Zone I

$305\left(t_{c}{ }^{I}\right)$. It is analogous to the Bejan number, which was developed for the pressure drop across a 306 channel [27].

\subsection{Diffusion Controlled}

Equations (18-21) and (23) can be simplified for diffusion controlled systems. The results

309 are summarized in the middle column of Table 2. If the Peclet number is very large, dispersion

310 effects are negligible. The zone velocities are controlled by Eq. (24). The resulting equation for

$311 D / F$ is presented in Eq. (25).

312 Equation (25) indicates that increasing the zone length $\left(N^{j} L_{c}\right)$ in any zone will decrease

$313 D / F$ by decreasing the effects from diffusion. The optimum column configuration is controlled

314 by the selectivity $(\alpha)$, diffusivity ratio $(\gamma)$, and size-exclusion factor ratio $(\lambda)$. For SEC-SMBs

315 with small dead volumes $(D V), \lambda$ is approximately equal to $\alpha$. If $\gamma$ is much larger than $\alpha$, Zone

316 IV should have more columns than Zone I, and Zone II should have more columns than Zone III.

317 A large value of $\gamma$ indicates that the fast solute has a lower diffusivity than the slow solute,

318 resulting in broader waves of the fast solute. This column configuration uses the extra columns in

319 Zones II and IV to better confine the trailing wave and advancing wave of the fast solute

320 (insulin), respectively. However, when $\gamma$ is large, the column configuration does not have a large 
321 impact on $D / F$ because the terms inside the parentheses in Eq. (25) are already relatively small.

322 The column configuration will have a larger impact on systems where $\alpha$ is larger than $\gamma$.

323 The denominator of Eq. (25) must be positive for SEC-SMB. Therefore there is a

324 minimum value of ${N_{D}}^{*}$ for the operation to be feasible, which is shown in Eq. (26).

$325 N D, \min *=115 \beta 2, \min N D * I I I \alpha 2 \lambda \gamma N I I I+\beta 1, \min N D * I I N I I$

326

327

328

329

330

331

332

333

334

335

336

337

338

339

340

For Eq. (20), the terms with Peclet numbers are negligible and the step time can be replaced by the definition of $N_{D}{ }^{*}$, resulting in Eq. (27)

$P R, i=\phi \delta 1 Y i C F, i \varepsilon p K s e, 1 D p, 1 N \rho p R p 2 N D, 1 \alpha-1-\phi \delta 115 N D, 1 \beta 2 I I I \alpha 2 \lambda \gamma N$

$I I I+\beta 1 I I N I I$

The second term in the brackets of Eq. (27b), Table 2, represents the loss of productivity due to diffusion effects compared to the productivity of an ideal system. For the productivity to be positive, the value of the second term in the in the bracket,

$115 N D * \beta 2 I I I \alpha 2 \lambda \gamma N I I I+\beta 1 I I N I I$, must be less than one. When the other parameters are fixed, there is a minimum $\gamma$, for the productivity to be positive.

For diffusion controlled systems, the step time is proportional to ${N_{D}}^{*}$. As ${N_{D}}^{*}$ increases, the loss of productivity due to diffusion effects decrease, but the step time increases. These competing effects result in a maximum in the productivity. Taking the partial derivative of Eq. (27b) with respect to $N_{D}{ }^{*}$ and setting the resulting equation equal to zero can solve for the $N_{D}{ }^{*}$ which achieves the maximum $P_{R}$. The result is shown in Eq. (28). 
345 Eq. (26) and Eq. (28) indicate that the $N_{D}{ }^{*}$ to achieve maximum productivity ( $N D$, max $\left.P R \star\right)$ is

346 about twice the value of the minimum ${N_{D}}^{*}$ for the SEC-SMB to be feasible $(N D, \min \star)$. The

347 maximum productivity, given by Eq. (28b), is inversely proportional to $R_{p}{ }^{2}$. Larger productivity 348 can be achieved with smaller particles, longer fractional zone length $\left(N^{j} / N\right)$ for Zones II and III, 349 or larger feed concentration, selectivity, size-exclusion factor, and diffusivity.

350 The optimum column configuration for maximum $P_{R}$ for diffusion controlled systems is 351 shown in Eq. (29).

$352 N I N=1 N$

$353 \quad N I I N=N I I I N \beta 1 I I \lambda \gamma \beta 2 I I I \alpha 2$

$354 \quad N I I I N=1-N I N-N I V N 1+\beta 1 I I \lambda \gamma \beta 2 I I I \alpha 2$

$355 \quad N I V N=1 N$

The maximum productivity configuration for diffusion controlled systems is not a

357 function of $N_{D}{ }^{*}$. As such, there is one column configuration which will have a larger productivity

358 than other configurations at every ${N_{D}}^{*}$. Once $\alpha, \lambda, \gamma, Y_{i}$, and $N$ are specified, the maximum

359 productivity configuration can be determined from Eq. (29). 
The values of $\beta$ 's for Zones II and III are often similar, so the maximum productivity

361 column configuration mainly depends on $\gamma / \alpha$, for small $D V(<0.02)$. A large $\gamma / \alpha$ indicates that

362 more columns should be placed in Zone II than Zone III to contain the wave of the fast solute.

363 The sharp wave of the slow solute in Zone III does not need as many columns when the

364 diffusivity of the slow solute is very large. A small $\gamma / \alpha$ means the reverse. More columns are

365 needed in Zone III to confine the spreading wave of the slow solute and Zone II does not need as

366 many columns because there is enough difference in the wave velocities to keep the trailing

367 wave of the fast solute confined in Zone II.

368 Equation (23) can be simplified since the term with the Peclet number is negligible.

369 Additionally, the port velocity can be replaced with a function of ${N_{D}}^{*}$. Rearrangement results in 370 Eq. (30a).

371 This group can be considered as a ratio of the diffusion time $\left(t_{D, I}\right)$ to pressure-limited

372 convection time through a column in Zone $\mathrm{I}\left(t_{c}{ }^{I}\right)$. Eq. (30a) indicates that $N \Delta \mathrm{P}$, diff decreases

373 with increasing $N_{D}{ }^{*}$. For fixed material properties, yields, and equipment parameters, Eq. (30a)

374 can be used to find the minimum $N_{D}{ }^{*}$ to satisfy the pressure limit. For a fixed diffusion time, a

375 large value of $N \Delta \mathrm{P}$, diff corresponds to a small pressure-limited convection time, or higher zone

376 velocities.

377 The $N_{D}{ }^{*}$ for maximum sorbent productivity can be found from Eq. (28). This value can

378 be used in Eq. (30a) to determine $N \Delta \mathrm{P}$, diff, $\max P R$, which is the combination of $\Delta P_{\max } R_{p}{ }^{4} L_{c}{ }^{2}$

379 necessary to achieve the maximum sorbent productivity, Eq. (30b).

$380 N \Delta \mathrm{P}, \mathrm{diff}, \max P R=\Delta \operatorname{Pmax} R p 437.5 \phi \mu D p, 1 L c 2=15 \phi 2 \alpha-12 \varepsilon p K s e, 12 \beta 2, \max \operatorname{PrIII} \alpha 2 \lambda$

$381 \gamma N I I I+\beta 1, \max \operatorname{Pr} I I N I I 1+\phi \delta 2 \phi \delta 2-\delta 1+\beta 2 I \alpha 22 \lambda \gamma N I \beta 2, \max \operatorname{Pr} I I I \alpha 2 \lambda \gamma N I I I+\beta 1, \max P$ 
384 For fixed material properties, yields, and column configuration, the right hand side of Eq. (30b)

385 is fixed. Thus, the value of $\Delta P_{\max } R_{p}{ }^{4} / L_{c}{ }^{2}$ for maximum productivity is fixed. For fixed particle

386 size and operating pressure (limited by equipment or resin material), there is only one column

387 length that can achieve the maximum productivity.

\subsection{Dispersion Controlled}

For axial dispersion controlled systems, $N_{D}{ }^{*}$ is very large and diffusion effects become

390 negligible. The dispersion controlled versions of Eqs. (18-21) and (23) are shown in the last

391 column of Table 2. The zone velocities for dispersion controlled systems are shown in Eq. (31).

392 The resulting equation for $D / F$ is presented in Eq. (32). For low Reynolds numbers, $P_{e b}{ }^{*}$ is

393 independent of port velocity. When $P_{e b}{ }^{*}$ is 50 or larger, the effects of dispersion on $D / F$ and $P_{R}$ 394 become negligible.

395 Similar to the diffusion controlled case, there is a minimum Peclet number for the SEC-

396 SMB operation to be feasible and it is given by Eq. (33).

$397 P e b, \min *=\beta 2, \min P e b \quad I I I \Gamma \min P e b I I I N I I I+\beta 1, \min P e b I I \Gamma m i n P e b I I N I I$

400 function $P_{e b}{ }^{*}$ using the Chung and Wen correlation for low Reynolds numbers $(\operatorname{Re}<10)$, for

401 which $E b j$ is related to $\varepsilon_{b}, R_{p}$, and $u O j$ as follows. [28]. 
403 Since $P_{e b}{ }^{I V}=L_{c} v / E b j$, Table 1, one can obtain Eq. (35).

$404 P e b I V=L c v 10 \varepsilon b R p u O I V$

405

$406 P e b *=\phi(\delta 2-\delta 1) L c v 10 \varepsilon b R p u O I V$

407

408 The $u O I V$ in Eq. (35) can be replaced by Eq. (31d) to obtain Eq. (36a) and rearranged to solve

409 for column length, Eq. (36b).

$410 P e b *=\phi \delta 2-\delta 11+\phi \delta 1 L c 10 \varepsilon b R p+\beta 1 I V N I V$

411 (36a)

$412 L c=10 \varepsilon b R p P e b * 1+\phi \delta 1 \phi \delta 2-\delta 1-\beta 1 I V N I V$

413

414 Equation (36b) can be substituted for the column length in Eq. (20), where the terms with

$415 N_{D}{ }^{*}$ are negligible, to obtain Eq. (37).

$416 P R, i=v Y i C F, i 10 N \varepsilon b R p P e b I V 1+\phi \delta 1-$

$417 \quad \beta 1 I V N I V \phi \rho p \phi \delta 1 \alpha-1-1$ PebIV $\beta 2 I I I \Gamma I I I N I I I+\beta 1 I I \Gamma I I N I I$

418 The $P_{e b}{ }^{*}$ for maximum $P_{R}(P e b, \max P R *)$ can be found by taking the partial derivative of 419 Eq. (36b) with respect to $P_{e b}{ }^{*}$ and setting the resulting equation to zero. The value of $420 P e b, \max P R \star$ can be determined from yields, material properties, and column configuration, see 
421 Appendix A3. The optimum column configuration for maximum $P_{R}$ for dispersion controlled 422 systems is shown in Eq. (38).

$423 \quad N I N=1 N$

$424 \quad N I I N=N I I I N \beta 1 I I \Gamma I I \beta 2 I I I \Gamma I I I$

$425 \quad$ NIIIN $=1-N I N-N I V N 1+\beta 1 I I \Gamma I I \beta 2 I I I \Gamma I I I$

$426 \quad N I V N=1 N$

427 Equation (23) can be simplified into Eq. (39) because the term with ${N_{D}}^{*}$ is negligible.

428 This group can be considered as a ratio of step time to pressure-limited convection time through

429 a column in Zone I. The column length in Eq. (39) can be replaced by Eq. (36b). Thus, for fixed

430 material properties and maximum pressure, the maximum port velocity at every $P_{e b}{ }^{*}$ can be

431 found. The value of $P e b, \max P R *$ (Appendix A3) can be substituted into Eq. (39) and

432 rearranged to find the port velocity for maximum $P_{R}$, Eq. (40).

$433 \nu \max P R=\Delta P \max R p 375 \phi 2 \mu \varepsilon b P e b * 1+\phi \delta 1 \phi \delta 2-\delta 1-\beta 1 I V N I V 1+\phi \delta 2+$

$434 \quad \beta 2 I \Gamma I \phi \delta 2-\delta 1 P e b, \max P R * N I$

435 The port velocity in Eq. (40) will achieve the maximum productivity when yields, material

436 properties, column configuration, viscosity, and maximum operating pressure are specified. If $R_{p}$

437 is fixed, there is only one $L_{c}$ that can satisfy $P e b, \max P R *$, as expected from Eq. (36b). Using

438 this port velocity to achieve maximum $P_{R}$, ensures that the minimum cost design will not be

439 pressure limited, as discussed in Section 3.6.1. 


\subsection{Optimization Using SSWD}

The values of the 15 decision variables $\left(Y_{i}, R_{p}, L_{c}, D V, N^{j}, \Delta P_{\max }, u_{0}^{j}\right.$, and $\left.v\right)$, which will

442 achieve minimum solvent, maximum productivity, or minimum cost can be found using the

443 SSWD. Overviews of $D / F$ and $P_{R}$ as functions of $N_{D}{ }^{*}$ and $P_{e b}{ }^{*}$ can be generated using Eq. (19)

444 and Eq. (20), respectively, for given input parameters (material properties, yields, column

445 configuration, dead volume, and maximum operating pressure). Minimum solvent consumption

446 and maximum productivity can easily be identified from these overviews. With given cost

447 functions (Appendix A4), the total cost surface can also be generated as a function of $N_{D}{ }^{*}$ and

$448 P_{e b}{ }^{*}$. By varying the input parameters of interest, the surfaces can be used to determine optimum

449 input parameters for minimum solvent consumption, maximum productivity, or minimum cost.

450 Optimization of the decision variables for maximum productivity becomes simpler for

451 the limiting cases already discussed in Sections 2.3 and 2.4. For diffusion controlled systems, the

452 value of $N_{D}{ }^{*}$ and the column configuration can be analytically determined using Eq. (28a) and

453 Eq. (29), respectively. For dispersion controlled systems, the value of $P_{e b}{ }^{*}$ and the column

454 configuration can be analytically determined using Eq. (A3.1a) and Eq. (38), respectively.

455 However, the values of $N_{D}{ }^{*}$ and $P_{e b}{ }^{*}$ for maximum productivity may not be achievable because

456 not all combinations of port velocity and column length can satisfy a given pressure limit, Eq.

457 (23).

458 For cost optimizations in this study, the costs are based on $\$ / \mathrm{kg}$ of product. Detailed cost

459 functions are given in Appendix A4. The overall separation cost (total cost) consists of

460 equipment cost, sorbent (or resin) cost, and solvent cost. Equipment cost is mainly controlled by

461 the maximum allowable pressure and total number of columns, Eq. (A4.2). Resin cost can be 
462 calculated from the sorbent productivity, Eq. (A4.3). Solvent cost can be calculated from solvent 463 consumption, Eq. (A4.4).

464 Optimization of decision variables for minimum cost can be achieved by evaluating the

465 total cost using the zone velocities and port velocity determined from the SSWD equations. For

466 the insulin example discussed in Section 3, four of the decision variables $\left(Y_{i}, R_{p}\right.$, and $\left.D V\right)$ are

467 fixed and the remaining 11 are optimized. The algorithm used to optimize the 11 decision

468 variables (column configuration, column length, operating pressure, zone velocities, and port

469 velocity) to achieve minimum cost is shown in Appendix A5, Fig. A5.1. The example algorithm

470 can easily be extended to optimize the remaining decision variables.

\section{$471 \quad 3.0$ Results}

472 The experimental $D / F$ and $P_{R}$ from three literature cases are compared to those of the

473 SSWD. Additionally, $D / F$ and $P_{R}$ are examined when one dimensionless group is varied at a

474 time. All other variables are held constant. The SEC-SMB for separating insulin from zinc

475 chloride was chosen as the example because the intrinsic parameters for the specific sorbent /

476 buffer system were verified experimentally [8]. The tandem SEC-SMB for insulin purification is

477 then optimized for minimum cost while satisfying an imposed maximum pressure drop per

478 column.

\section{$479 \quad 3.1$ Comparisons of D/F and $P_{R}$ of Literature Designs with Those of SSWD}

480 The three literature SEC-SMB separations analyzed in this study are polyethylene glycol

481 fractionation by molecular weight (Case 1, Fig. 4) [12], myoglobin separated from bovine serum

482 albumin (BSA) (Case 2, Fig. 5) [29], and insulin separated from zinc chloride (Case 3, Fig. 6)

483 [8]. A brief summary of the three cases is presented in Table 3 with the complete set of material 
484 properties and design parameters in Appendix B. The operating parameters of Cases 1 and 2

485 reported in the literature were obtained using the Triangle theory. The operating parameters of

486 Case 3 were designed using the SWD method at the specific ${N_{D}}^{*}$ of 8.9 (Table 3).

487 The material properties, equipment parameters, and yields for each case reported in the

488 literature were used in the SSWD equations to generate $D / F$ and $P_{R}$ for a wide range of operating

489 parameters. For Case 1 , both $N_{D}{ }^{*}$ and $P_{e b}{ }^{*}$ are very large, making it a nearly ideal system. For

490 Case 2, both $N_{D}{ }^{*}$ and $P_{e b}{ }^{*}$ are relatively small, so both diffusion and dispersion effects are

491 important. For Case $3, N_{D}{ }^{*}$ is relatively small while $P_{e b}{ }^{*}$ is relatively large, making it a diffusion

492 controlled system.

493 Figure 4 a shows the $D / F$ results from SSWD for Case 1, which is a three-zone open-loop

$494 \mathrm{SMB}$. The minimum $D / F$ from SSWD is about 6.5 because there is no recirculation of the

495 solvent. The difference between the three curves is due to the yield requirements, Table 3.

496 Because the $N_{D}{ }^{*}$ value for each of the three runs (1A-1C) is over 4,000, diffusion effects in Case

4971 are negligible. The solvent consumption for the Case 1 experiments could have been reduced

498 by about $50 \%$ if the operating parameters had been obtained from the SSWD method, Figs. $4 \mathrm{a}$, $4997 \mathrm{a}$.

$500 \quad$ Figure $4 \mathrm{~b}$ shows the $P_{R}$ results from SSWD for Case 1 . Because the $N_{D}{ }^{*}$ values are so 501 large, the $P_{R}{ }^{*}$ is very low. The results from SSWD show that $P_{R}$ could increase over 100 fold if 502 the operating parameters were designed based on ${N_{D}}{ }^{*} \sim 4,600$.

503 Figure 5 compares the SSWD results with the literature results for Case 2. The 504 experiments in the literature were operated at a relatively low ${N_{D}}{ }^{*}(\sim 0.4)$. The curves generated 505 by the SSWD do not extend to lower $N_{D}{ }^{*}$ values because assumptions used to obtain Eq. (1) do 506 not hold in the low yield and low $N_{D}{ }^{*}$ regime [14]. In Fig. 5a, the $D / F$ curve for $2 \mathrm{E}$ is 
507 significantly higher than the other curves because the yield for component 1 (BSA) is very large 508 (99.8\%) and the yield for component 2 (Myoglobin) is very low (40.5\%) compared to the other

509 experiments. The solvent consumption for these experiments could have been reduced 2-9 fold if 510 the operating parameters were designed using SSWD at the same $N_{D}{ }^{*}$, Fig. $7 \mathrm{a}$.

$511 \quad$ Figure $5 \mathrm{~b}$ shows the $P_{R}$ results from SSWD for Case 2. The $P_{R}$ curves generated by the 512 SSWD do not extend to low ${N_{D}}{ }^{*}(<0.3)$ because of the low yield specifications. Because the $N_{D}{ }^{*}$ 513 values for the literature are lower than those of Case 1, the productivities are much larger. The 514 productivity values for Case $2 \mathrm{~A}-2 \mathrm{E}$ are in the same order of magnitude as that of the SSWD at 515 the same $N_{D}{ }^{*}$. However, the $P_{R}$ for Case 2A-2E could have been increased 4-6 fold if the 516 operating parameters were designed using SSWD at the same $N_{D}{ }^{*}$, Fig. $7 \mathrm{~b}$.

517 Since the operating parameters for Case 3 were designed using SWD, $D / F$ and $P_{R}$ of are 518 the same as those from SSWD at the same ${N_{D}}^{*}$, column configuration (2-3-3-2), and yields 519 (99.7\%) Figs. 6-7.

520 The SSWD works for ideal systems, diffusion controlled systems, or systems where both 521 diffusion and dispersion are significant. For given material properties, yields, and equipment 522 parameters, SSWD can quickly generate an overview of $D / F$ and $P_{R}$ over a wide range of 523 operating parameters. The conditions for maximum $P_{R}$ or small $D / F$ can be easily identified from 524 such figures. The comparisons with literature results show that all three cases could have been 525 improved by orders of magnitude using the SSWD method to reduce $D / F$ and/or increase $P_{R}$. 526 Without the overview provided by the SSWD, it would be challenging to explore the multi527 dimensional SEC-SMB design space with simulations or experiments. 

crystallization, SEC is used to separate high molecular weight proteins (HMWP) and zinc chloride $\left(\mathrm{ZnCl}_{2}\right)$ from insulin [8]. This batch chromatography produces $99 \%$ pure insulin with

$53289 \%$ yield. In 2002, Xie et al. designed and experimentally verified a tandem SEC-SMB system

533 (two SMBs in series) to obtain 99\% pure insulin with 99\% yield.

The first ring of the tandem SMB (Ring A) separates the HMWP (fast solute) from

535 insulin (slow solute). No constraints are placed on the zinc chloride, which means that zinc 536 chloride is distributed throughout the entire SMB to reduce the impurity to be removed in Ring 537 B. There is significant insulin fronting due to dimerization reactions that occur in Zone III of 538 Ring A. This fronting can be accounted for by assuming that the axial dispersion coefficient in 539 Zone III is 40 times the value predicted by the Chung and Wen correlation [8]. Because of this 540 large axial dispersion, both diffusion and dispersion effects are important for Ring A. The extract 541 from Ring A is then sent to the second SMB (Ring B) to remove the zinc chloride.

542 Ring B separates zinc chloride (slow solute) from insulin (fast solute). Because of 543 dilution from Ring A, the insulin fronting is insignificant in Ring B. Thus, Ring B is a diffusion 544 controlled system. The solutions for $D / F$ and $P_{R}$ are calculated according to Table 2 . The 545 intrinsic material properties and yields were obtained from Xie et al., 2002, Table 4. The 546 dimensionless parameters in Table 4 are fixed for all figures unless specified otherwise. Ring A

547 will later be used as a cost optimization example (Section 3.6), where both diffusion and 548 dispersion are significant. Ring B is used for the parametric studies in the following sections. 549 The tandem SMB requires significantly less solvent and less sorbent than the batch SEC, Table $550 \quad 5$. 


\subsection{Parametric Studies - Diffusion Controlled}

For $D / F, P_{R}$, and pressure limit curves in this section, the values for $\alpha, \lambda, \gamma, \delta, \phi$, and

553 yields are reported in Table 4 and are fixed unless noted otherwise. For $D / F$ curves, $t_{s}, R_{p}$, and

$554 D_{p, i}$, are allowed to vary with changing $N_{D}{ }^{*}$. For $P_{R}$ and pressure limit curves, $\varepsilon_{p}, \varepsilon_{b}, K_{s e, i}, D_{p, i}, N$,

555 feed concentration $\left(C_{F, i}\right)$, packing density $\left(\rho_{b}\right)$, and $R_{p}$, are fixed at the values reported in Table 4,

556 unless noted otherwise. For $P_{R}$ curves, $t_{s}$ is allowed to vary with changing $N_{D}{ }^{*}$. For the pressure

557 limit curves, viscosity $(\mu)$ and maximum pressure $\left(\Delta P_{\max }\right)$ are fixed at the values reported in

558 Table 4 and $L_{c}$ is fixed at $13.5 \mathrm{~cm}$.

\subsection{1 $N_{D}^{*}$ and Column Configuration}

Figure 6 also shows how column configuration affects solvent consumption and sorbent

561 productivity for diffusion controlled systems. Since most industrial applications have a fixed

562 number of columns or valves, it is more useful to determine the optimum distribution of

563 columns, or column configuration, for a given total number of columns. Three column

564 configurations with 12 columns total are plotted for comparison purposes: minimum $D / F(2-4-2-$

565 4), maximum productivity (2-6-2-2), and an equal distribution (3-3-3-3).

$566 \quad$ Figure 6a illustrates the relation between $N_{D}{ }^{*}$ and $D / F$ for the three column

567 configurations. As $N_{D}{ }^{*}$ increases, the step time increases relative to the diffusion time, which

568 means that diffusion becomes less controlling and the system approaches an ideal system with

$569 D / F$ equal to one. Solvent consumption decreases slowly after $N_{D}{ }^{*}$ increases beyond 2 for this

570 system. As $N_{D}{ }^{*}$ decreases, the denominator of Eq. (25) approaches zero, resulting in a sharp rise

571 in $D / F$ near $N D, \min *$, which corresponds to the minimum relative diffusion time required for

572 any separation to occur. The vertical line is based on the pressure limits of the system (1.5 psi)

573 [9], and the column length of $13.5 \mathrm{~cm}$, which is the optimal column length to achieve the lowest 
574 total cost Ring B (Section 3.6). This line can be found by solving Eq. (30) for $N_{D}{ }^{*}$ because $N_{A P, \text { diff }}$

575 is fixed for fixed material properties, yields, and equipment parameters. $N_{D}{ }^{*}$ values to the right of 576 this will give port velocities, or interstitial velocities, which do not exceed the pressure limit.

577 In this example, diffusivity ratio $(\gamma=7.21)$ is much larger than the selectivity $(\alpha=1.34)$.

578 Because the terms inside the parentheses in Eq. (25) are already relatively small, the column

579 configuration does not have a large impact on $D / F$.

580 The effect of $N_{D}{ }^{*}$ on $P_{R}$ is presented in Fig. 6b. There is a minimum $N_{D}{ }^{*}$ for $P_{R}$ to be 581 positive, $N D, \min *(\sim 0.1)$, which is the same $N D, \min *$ for $D / F$. Increasing $N_{D}{ }^{*}$ from $N D$,

$582 \min *$ to $N D, \max P R *$ will increase $P_{R}$, as expected from Eq. (27b). Increasing $N_{D}{ }^{*}$ means

583 sharper concentration waves, which mean more effective utilization of the sorbent, or higher 584 sorbent productivity. The $P_{R}$ curve peaks near $N_{D}{ }^{*}=0.2$, Eq. (28). This ${N_{D}}{ }^{*}$ corresponds to $N_{D, 1}$ $585 \sim 1$, Eq. (16), where the step time is approximately equal to the diffusion time. Increasing $N_{D}{ }^{*}$ 586 after this point reduces $P_{R}$ because the increase in step time is more significant than the reduced 587 wave spreading. In general, the maximum $P_{R}$ for SEC-SMBs occurs at $N_{D, 1} \sim 1$, as evidenced by 588 all three literature cases.

589 Figure $6 \mathrm{~b}$ also shows how column configuration significantly affects the maximum $P_{R}$.

590 Because $\gamma / \alpha$ is about 5.4, Eq. (29) gives 2-6-2-2 as the column configuration for maximum 591 productivity. For ${N_{D}}^{*}$ larger than 2 , the column configuration does not significantly affect the 592 productivity.

593 The pressure limit for a column length of $13.5 \mathrm{~cm}$ is again shown by the vertical line, 594 indicating that the theoretical maximum productivity is not achieved since the pressure limit, 595 column length, particle size, and column configuration are fixed. The productivity for the lowest 
cost system is less than $15 \%$ of the theoretical maximum productivity for the 2-6-2-2 column

597 configuration. requirement increases the values of the four $\beta$ terms in Eq. (25), resulting in the increase of the

\subsubsection{Particle Size and Pressure Limit}

$P_{R}$ is strongly affected by particle size, as seen in Fig. 8a. From Eq. (27b), it is obvious that $P_{R}$ is inversely proportional to $R_{p}{ }^{2}$ for a fixed $N_{D}{ }^{*}$. However, reducing $R_{p}$ also increases the pressure drop across a column $(\Delta P)$. As discussed in Section 2.3, Eq. (28) and Eq. (30) can be used to determine the combination of pressure limit, column length, and particle size $\left(\Delta P_{\max } R_{p}{ }^{4} / L_{c}{ }^{2}\right)$, which is needed to achieve the maximum productivity.

In Fig. $8 \mathrm{~b}, \Delta P / L_{c}{ }^{2}$ is plotted for a wide range of $R_{p}(5-500 \mu \mathrm{m})$ for fixed column configuration (3-3-3-3) and other material properties (Table 4). The yield for each solute is varied from $90 \%$ to $99.9 \%$. This result shows that the pressure drop rapidly increases when $R_{p}$ is below $50 \mu \mathrm{m}$. For a $10 \mathrm{~cm}$ column packed with $R_{p}=50 \mu \mathrm{m}$, the pressure drop required to realize the maximum productivity is $10 \mathrm{psi}$. For $R_{p}=25 \mu \mathrm{m}$, the productivity can be four times higher (Fig. 8a), but the pressure drop will be about 1,000 psi, which requires high pressure equipment. Conversely, for high pressure SMB equipment with a maximum operating pressure of 1,500 psi, one can find the combination of particle size and column length to maximize productivity. The trade-off between pressure and productivity can be optimized if appropriate cost functions are known. numerator and the decrease of the denominator. Thus, increasing yield results in increasing $D / F$. However, the increase in $D / F$ is not very significant until yield is larger than $99 \%$. The increase 
619 after $99 \%$ is more significant for small ${N_{D}}^{*}(<1)$. Increasing ${N_{D}}{ }^{*}$ reduces the dependence of $D / F$

620 on yield. There is a sharp increase near $100 \%$ yield because significantly more solvent is

621 required to confine the very low concentration portions of the waves in their respective zones.

622 The effect of yield on $P_{R}$ is presented in Fig. 9b. Increasing yield from $90 \%$ does not

623 significantly change $P_{R}$ until after yield exceeds 99\%. From Eq. (27), it can be seen that

624 increasing the values of the $\beta$ 's in the denominator decreases $P_{R}$, but the yield outside the

625 brackets in the numerator mitigates this effect until the yields are very high. Increasing yield

626 requirement means more separation between the solute bands, resulting in less column utilization

627 or lower $P_{R}$. The productivity approaches zero near $100 \%$ yield because a very small feed flow

628 rate is required for the separation. Increasing $N_{D}{ }^{*}$ decreases the dependence of $P_{R}$ on yield.

\subsubsection{Retention Factors and Selectivity}

Because $N_{D}{ }^{*}$ is dependent on selectivity and retention factors, $N_{D, 1}$ was fixed at 6.5 ,

631 which corresponds to the lowest cost design for Ring B (see Section 3.6.1). Solvent consumption

632 is plotted against $\alpha$ in Fig. 10a. Increasing $\alpha$ always decreases $D / F$ because there is an increasing

633 difference in wave velocities but there is little gain when $\alpha$ is larger than 1.5. There is a vertical

634 asymptote at $\alpha$ equal to one because there would be no difference in the wave velocities of the

635 two species and no separation could occur. The effect of the retention factors $(\delta)$ can also be seen

636 in Fig. 10a. The lines for $\delta_{l}$ stop at different $\alpha$ 's because there is a maximum $\delta_{2}$ in SEC. When a

637 solute accesses all the pore space, the maximum size-exclusion factor is one and the maximum $\delta$

638 is equal to the particle porosity if $D V$ is negligible, Eq. (4). The $D / F$ values are higher for larger

$639 \delta$ 's because more solvent is needed for the solutes to diffuse out of the particles at the same $\alpha$.

640 Increasing $\alpha$ always increases the $P_{R}$, as shown in Fig. 10b. Increasing $\alpha$ is shown as

641 increasing $\delta_{2}$ for a fixed $\delta_{1}$. The more the slow solute can access the pore volume, the more 
642 efficiently the sorbent particles are used. Therefore, a larger retention factor for the slow solute

643 provides a higher productivity at the same selectivity. The $P_{R}$ linearly increases with selectivity

644 because the $P_{R}$ for an ideal system is linearly dependent on selectivity, as are the correction terms

645 for non-ideal SEC systems with negligible $D V$, Eq. (27a).

\subsubsection{Dead Volume Effect on Retention Factors and Selectivity}

648 dispersion can be plug flow (no dispersion), Taylor dispersion, or that of a completely stirred

649 tank. Since they are equipment dependent, they are not considered in this study. Only the effect

650 of $D V$ on the retention factors, and subsequently selectivity, is discussed below.

652 dependent on $\delta$, Eq. (16), constant $N_{D, 1}$ curves are shown in Fig. 11. Dead volume can

653 significantly increase $D / F$, Fig. 11a. From Eq. (25), it is clear that increasing $\delta$ values increases

$654 D / F$ for diffusion controlled systems. However, the effect of $D V$ can be significantly reduced at

655 larger $N_{D, 1}$. Because the difference in the retention factors is unaffected by $D V, D / F$ is unaffected

656 by $D V$ at large $N_{D, 1}$, Eq. (13). Dead volumes less than $2 \%(D V<0.02)$ do not significantly

657 increase $D / F$ for this system.

658 Increasing the $D V$ of the system reduces the selectivity, which in turn reduces the

659 productivity, Fig. $11 \mathrm{~b}$. It can be seen from Eq. (14) that increasing $N_{D, I}$ can reduce the impact of

$660 D V$ because the difference in retention factors is not affected by $D V$. However, the productivity

661 also decreases with increasing $N_{D, l}$. Dead volumes less than $2 \%$ do not significantly affect the

662 productivity of the SEC-SMB. 


\subsubsection{Diffusivity Ratio}

The effect of diffusivity ratio $(\gamma)$ on $D / F$ is explored in Fig. 12a. As $\gamma$ increases, $D / F$

665 decreases when $N_{D}{ }^{*}$ is a fixed parameter (i.e. $N_{D, l}$ is fixed). Increasing $\gamma$ with a fixed $N_{D, l}$ is

666 equivalent to increasing $D_{p, 2}$. By increasing the diffusivity of the slow solute, the concentration

667 waves become sharper and thus require less solvent to prevent the waves from spreading into

668 different zones. There is a minimum value of $\gamma$ for the system to be feasible, as discussed in

669 Section 2.3. For $\gamma$ greater than three, $D / F$ does not change significantly. The $D / F$ dependence on

$670 \gamma$ is larger at low ${N_{D}}^{*}$, where diffusion effects are more significant.

671 The $P_{R}$ curves always increase with increasing $\gamma$, until $P_{R}$ reaches a plateau. The waves

672 become sharper, resulting in increased column utilization or sorbent productivity, Fig. 12b. As

673 discussed in Section 2.3, there is a minimum $\gamma$ for $P_{R}$ to be positive. The $P_{R}$ does not change

674 significantly after $\gamma$ exceeds 3 . Increasing $N_{D}{ }^{*}$ reduces $P_{R}$, and also reduces the dependence of $P_{R}$ 675 on $\gamma$.

676 Figures 10 and 12 show that large $\alpha$ and large $\gamma$ both decrease $D / F$ and increase $P_{R}$. A

677 large ratio of $\gamma / \alpha$ can also decrease $D / F$ and increase $P_{R}$, as shown in Eqs. (25) and (27a). If $\gamma / \alpha$

678 is small, increasing the length of Zones I and III can have a similar effect on $D / F$ as increasing

$679 \gamma / \alpha$, Eq. (25). Increasing the length of Zone III also increases $P_{R}$, Eq. (27a).

\subsubsection{Phase Ratio}

681 For diffusion controlled systems, $D / F$ is independent of the phase ratio ( $\phi$ ), Eq. (25),

682 because $N_{D}{ }^{*}$ is independent of $\phi$, Table 1 . The $P_{R}$ is also independent of $\phi$, Eq. (27). 


\subsection{Parametric Studies - Dispersion Controlled}

As shown in Table 2, $D / F, P_{R}$, and $N_{\Delta P}$ are functions of $\Gamma$. To obtain values for $\Gamma$, axial

685 dispersion coefficients were estimated using the Chung and Wen correlation for low Re, Eq.

686 (34). For $D / F, P_{R}$, and pressure limit curves in this section, the values for $\alpha, \delta$, $\phi$, and yields are

687 reported in Table 4 and are fixed unless noted otherwise. For $D / F$ curves, $L_{c}$ and $R_{p}$ are allowed

688 to vary with changing $P_{e b}{ }^{*}$. For $P_{R}$ and pressure curves, the values of $\varepsilon_{b}, N, C_{F, i}, \rho_{p}$, and $R_{p}$, are

689 given in Table 4, unless noted otherwise. The port velocity for $P_{R}$ and pressure curves is fixed at

$6901 \mathrm{~cm} / \mathrm{min}$. For $P_{R}$ curves, $L_{c}$ varies with changing $P_{e b}{ }^{*}$. For the pressure curves, $\mu$ and $\Delta P_{\max }$ are

691 fixed at the values reported in Table 4 and $L_{c}$ is fixed at $13.5 \mathrm{~cm}$.

\subsection{1 $P_{e b}{ }^{*}$ and Column Configuration}

694 system to be feasible, as predicted by Eq. (33). The $D / F$ decreases with increasing $P_{e b}{ }^{*}$ because

695 axial dispersion effects decrease. These trends are supported by Eq. (32). The $D / F$ does not

696 change much after a $P_{e b}{ }^{*}$ of $50\left(L_{c} / R_{p} \sim 900\right.$ for the given $\phi$ and $\delta$, Eq. (35a)), when the

697 dispersion effects start becoming negligible.

698 Figure 13a also shows the effect of column configuration on $D / F$. From Eq. (32), it is

699 apparent that increasing the zone length of any zone will decrease $D / F$. Since the $\Gamma$ values for the

700 four zones are not very different from each other, the column configuration for minimum $D / F$ is

701 an equal distribution (3-3-3-3). However, the $D / F$ curve for the minimum $D / F$ configuration is

702 only slightly better than the $D / F$ curve for the maximum $P_{R}$ configuration (2-4-4-2).

703 For a fixed particle size, increasing $P_{e b}{ }^{*}$ is equivalent to increasing the column length.

704 Because maximum pressure drop and port velocity are fixed, the maximum column length can be 
705 illustrated in Fig. 13 as a vertical line. The vertical line represents the maximum $P_{e b}{ }^{*}$ allowed by 706 the pressure limit. Values to the left of the line will satisfy the pressure limit.

707 The effect of $P_{e b}{ }^{*}$ on $P_{R}$ is illustrated in Fig. 13b and can be seen from Eq. (35). There is a 708 minimum $P_{e b}{ }^{*}$ for the SMB to have a positive $P_{R}$, which is the same minimum $P_{e b}{ }^{*}$ for $D / F$. $P_{R}$ 709 increases with increasing $P_{e b}{ }^{*}$ rapidly at first, then peaks, and then slowly decreases. Increasing

$710 P_{e b}{ }^{*}$ decreases the effects from axial dispersion. The maximum $P_{R}$ occurs around a $P_{e b}{ }^{*}$ of 6 . The

711 effects of column configuration can also be seen in Fig. 13b. Longer Zones II and III can

712 increase $P_{R}$ significantly, as expected from Eq. (37), but there is no effect at large $P_{e b}{ }^{*}(>50)$

713 because the dispersion effects become negligible.

\subsubsection{Yield}

The effect of yield requirement on $D / F$ for dispersion controlled systems is shown in Fig.

716 14a. Increasing yield requirement always increases $D / F$ because of the extra solvent needed to

717 confine the low concentration portions of the waves. This trend is similar to that for diffusion

718 controlled systems. $D / F$ does not change much until after yield exceeds $99 \%$. There is a sharp

719 increase near $100 \%$ yield because significantly more solvent is required to confine the very low

720 concentration portions of the waves in their respective zones. Increasing $P_{e b}{ }^{*}$ decreases the effect

721 of yield on $D / F$ because dispersion effects decrease.

722 The effect of yield on $P_{R}$ is shown in Fig. 14b. Productivity does not significantly change

723 with yield until it exceeds $99 \%$. Increasing $P_{e b}{ }^{*}$ decreases the effect of yield on $P_{R}$, but also

724 decreases $P_{R}$. To approach $100 \%$ yield, the feed flowrate must be significantly reduced, resulting 725 in very small $P_{R}$. 


\subsubsection{Retention Factors and Selectivity}

727 Because $P_{e b}{ }^{*}$ is a function of retention factors and selectivity (Table 1), Fig. 15 is

728 generated using a constant Peclet number in Zone IV $\left(P_{e b}{ }^{I V}=50\right)$. At very low selectivities $(\alpha \sim$

729 1), very large amounts of solvent are required to achieve separation, Fig. 15a. Increasing $\alpha$

730 decreases the amount of solvent required for the separation because less solvent is needed to

731 confine the concentration waves in their respective zones. Larger values of retention factors at

732 the same $\alpha$ result in lower $D / F$. This result can be explained by Eq. (17) and Eq. (32) because

733 increasing the retention factors increases $P_{e b}{ }^{*}$, which decreases $D / F$. The lines for different

734 retention factors end at different $\alpha$ 's because of the limit on the maximum value of a retention

735 factor in SEC.

736 The impact of $\alpha$ on $P_{R}$ can be seen in Fig. 15b. Productivity increases linearly with

737 increasing $\alpha$, Eq. (36a). Increasing $\delta_{1}$, while maintaining the same $\alpha$, results in a larger $P_{R}$

738 because a larger amount of the pore phase of the sorbent particles is being accessed by the

739 solutes, increasing the column utilization and productivity.

\subsubsection{Phase Ratio}

741 The solvent consumption $(D / F)$ as a function of $P_{e b}{ }^{*}$ is given in Eq. (32). For constant

$742 P_{e b}{ }^{*}, D / F$ has only a weak dependence on $\phi$ because the axial dispersion coefficient ratios $\left(I^{j}\right)$ are

743 weak functions of $\phi$, Eq. (A2.1). The phase ratio only affects $D / F$ for low $P_{e b}{ }^{*}(<20)$, figure not

744 shown. Increasing $\phi$ increases $P_{R}$ for dispersion controlled systems, according to Eq. (36b). The

745 term $\left(1+\phi \delta_{l}\right) / \phi$ does not change significantly with increasing $\phi$, but decreasing the $\varepsilon_{b}$ in the

746 denominator outside the brackets increases $P_{R}$, figure not shown. 


\subsection{Comparison of Diffusion Controlled and Dispersion Controlled Systems}

Diffusion controlled systems and dispersion controlled systems are very similar in how

$749 D / F$ and $P_{R}$ vary with the dimensionless groups. Increasing $N_{D}{ }^{*}\left(\right.$ or $\left.P_{e b}{ }^{*}\right)$ decreases $D / F$ while $P_{R}$

750 increases to a maximum and then decreases. Increasing $\alpha$ decreases $D / F$ and increases $P_{R}$ while

751 increasing yield increases $D / F$ and decreases $P_{R}$. Small retention factors favor low $D / F$ and high

$752 P_{R}$ for diffusion controlled systems, whereas large retention factors are better for dispersion

753 controlled systems. Increasing $\gamma$ decreases $D / F$ and increases $P_{R}$ for diffusion controlled systems,

754 but has no effect on dispersion controlled systems. Increasing $\phi$ increases $P_{R}$ for dispersion

755 controlled systems, but has no effect on $D / F$ or $P_{R}$ for diffusion controlled systems. Maximum

$756 P_{R}$ for diffusion controlled systems can be achieved when the diffusion time, step time, and

757 pressure-limited convection time are matched. For dispersion controlled systems, the maximum

$758 P_{R}$ occurs when the axial dispersion time is about 10 times the step time and 50 times the

759 convection time.

\section{$760 \quad 3.6$ Cost Optimization of SEC-SMB for Insulin Purification}

761 The production scale was chosen to be 5,000 kg insulin / year and the optimization results

762 are reported in Table 5. Low pressure SMB $(<150$ psi per zone) equipment was chosen because

763 at this production scale, the equipment cost is dominating and high pressure equipment would be

764 even more expensive. The sorbent was chosen to be the same as the sorbent used in the batch

765 purification of insulin because the material properties are known and the performance of

766 optimized SEC-SMB can be compared with that of batch SEC. For this particular sorbent,

767 Sephadex G50, the maximum pressure drop per column is limited to $1.5 \mathrm{psi}$. The total dead

768 volume was kept at $1.9 \%$ of the total packing volume because $D V$ less than $2 \%$ does not

769 significantly affect $D / F$ or $P_{R}$, Fig. 11 . The yield specifications for both rings were set at $99 \%$ 
770 because Fig. 9 shows that the yields do not significantly affect $D / F$ or $P_{R}$ unless they are

771 specified over $99 \%$. The potential impact of optimizing particle size on total cost is small $(<2 \%)$

772 for this sorbent, and will be discussed in the following section. For these reasons, four decision

773 variables $\left(Y_{i}, R_{p}, D V\right)$ were fixed, while the remaining 11 decision variables $\left(L_{c}, N^{j}, \Delta P_{\max }, u_{0}^{j}\right.$,

774 and $v$ ) were optimized for minimum cost. Optimization of the 11 decision variables for one ring

775 takes less than one minute on a laptop.

\subsubsection{Ring B Cost Optimization}

Ring B is diffusion controlled whereas both diffusion and dispersion effects are important

778 in Ring A. For this reason, the optimization of Ring B is discussed first. Because the material

779 properties are fixed, the total cost for Ring B can be plotted versus $N_{D}{ }^{*}$ as a single 2-D curve,

780 Fig. 16. Cost functions for solvent, sorbent, and equipment are presented in Appendix A4. Unit

781 equipment cost does not vary with $N_{D}{ }^{*}$ for fixed $N^{j}$ and $\Delta P_{\max }$. At low $N_{D}{ }^{*}(<0.2, N D$,

$782 \max P R \star$ ), sorbent and solvent costs are high because $D / F$ is large and productivity is low, Fig.

783 6. The costs decrease with increasing $N_{D}{ }^{*}$ as $D / F$ decreases and productivity increases. When

$784 N_{D}{ }^{*}$ is larger than $N D, \max P R *$, the sorbent cost increases with $N_{D}{ }^{*}$ because the productivity

785 decreases. This increase in the sorbent cost causes a minimum in the total cost curve. This

786 minimum, however, does not satisfy the pressure limit for this system. As a result, the lowest

787 cost design allowed by the pressure is at ${N_{D}}{ }^{*} \sim 2$. At this ${N_{D}}^{*}$, column configuration has little

788 effect on $D / F$ or productivity, Fig. 6 . However, the equipment cost can be lowered by using

789 fewer columns. Thus, the optimum column configuration is 2-2-2-2 if a minimum of two

790 columns per zone is required. 
If production scales are much larger than that of insulin, the unit equipment cost can be a

792 small fraction of the total separation cost. In these cases, optimization of the material properties

793 (especially $\left.R_{p}\right)$ and equipment parameters $\left(L_{c}, \Delta P_{\max }\right)$ can significantly reduce the separation

794 cost. For diffusion controlled systems, one can find the optimal combination of $\left(\Delta P_{\max } R_{p}^{4} / L_{c}{ }^{2}\right)$,

795 using Eq. (30a), such that the minimum cost design is feasible. One can use $N D$, max $P R$ *, Eq.

796 (28a), in Eq. (30a) to obtain $N \Delta \mathrm{P}$, diff, $\max P R$. With $\phi$ and viscosity $(\mu)$, the value of

$797\left(\Delta P_{\max } R_{p}{ }^{4} / L_{c}{ }^{2}\right)$ can be calculated. Because the value of $N_{D}{ }^{*}$ corresponding to the minimum cost

798 design is always greater than, or equal to, $N D$, max $P R \star$, designing $\Delta P_{\max } R_{p}{ }^{4} / L_{c}{ }^{2}$ for maximum

$799 P_{R}$ will always satisfy the pressure limit at the minimum cost design.

$800 \quad$ For dispersion controlled systems, Eq. (39) can be used to find the minimum $N_{\Delta P}$ at the

$801 P_{e b}{ }^{*}$ value corresponding to the minimum cost design. The value of $N_{\Delta P}$ can then be used to find a

802 value of $\left(\Delta P_{\max } R_{p}{ }^{2} / L_{c} v\right)$, which ensures that the minimum cost design satisfies the pressure limit.

803 For systems in which both diffusion and dispersion effects are significant, Eq. (23) can be used

804 in a similar manner.

805 The calculated cost of Ring B based on the experimental design reported by Xie et al., 8062002 is about $\$ 6$ higher than the minimum cost found in this study. Their operating conditions

807 were based on SWD for a fixed column length, $13.7 \mathrm{~cm}$, and 10 columns were used instead eight 808 columns, the optimum number of columns obtained from SSWD. The switching time was also 809 longer than the optimum switching time, resulting in ${N_{D}}^{*}=8.9$. The calculated cost falls close to 810 the SSWD prediction for $N_{D}{ }^{*}=8.9$, Fig. 16 . The difference in total cost is mainly due to the 811 difference in the total number of columns. 


\subsubsection{Ring A Cost Optimization}

813 Because both diffusion and dispersion effects are important for Ring A, the total cost

814 should be plotted against $N_{D}{ }^{*}$ and $P_{e b}{ }^{*}$ as a 3D surface. However, the total cost surface is concave

815 up and cannot be easily viewed. As such, the inverse of the total cost is plotted against $N_{D}{ }^{*}$ and

$816 P_{e b}{ }^{*}$ in Fig. 17a. Since the z-axis is the inverse of total cost, the maximum point of the surface

817 corresponds to the minimum cost design.

818 The grey surface represents the pressure limit and divides the cost surface into feasible

819 and infeasible regions. $N_{D}{ }^{*}$ lower than the pressure surface and $P_{e b}{ }^{*}$ larger than the surface would

820 violate the maximum pressure constraint. Because the system is limited by pressure, the

821 achievable minimum cost point is ${N_{D}}^{*}=14.9$ and $P_{e b}{ }^{*}=560$, which is marked on a contour plot

822 of the surface, Fig. 17b. The lines in Fig.17b represent constant total cost values projected onto

823 the $N_{D}{ }^{*}$ and $P_{e b}{ }^{*}$ plane. The black dashed line is the pressure limit. The region below the black

824 line is infeasible. A summary of the optimized costs and equipment parameters are shown in

825 Table 5.

826 Table 5 compares batch SEC production of insulin and two different SEC-SMB systems

827 based on the same sorbent. The SMB design of Xie in 2002 was based on the operating

828 parameters from the SWD method for a fixed column length of $13.7 \mathrm{~cm}$. A cost optimized SMB

829 design for both Ring A and Ring B was found using the SSWD equations, Table 5. The cost

830 optimized design reduced the overall separation cost by $16 \%$ from the design of Xie et al. Both

831 SMBs are significantly lower in cost than the batch method. The optimized SMB reduces the

832 solvent consumption from batch SEC by $66 \%$, increases the productivity by 34 times, and

833 reduces the cost by $62 \%$. 


\subsubsection{Ring B Material Property and DV Sensitivity}

835

836

837

838

839

840

841

842

843

844

845

846

847

848

849

850

851

852

853

854

855

856

The cost optimization shown in Table 5 is based on the material properties, pressure

limit, and dead volume of the experimental system of Xie et al. Column length and configuration are optimized to reduce the unit separation cost. If the material properties, pressure limit, and dead volume are allowed to vary, the SSWD can further reduce the separation cost. The effects

of changing each of the equipment parameters and material properties on solvent cost and sorbent cost are shown in Table 6.

Increasing the pressure limit from 1.5 to 50 psi per column moves the pressure limit line in Fig. 16 to a lower $N_{D}{ }^{*}$, allowing the system to be designed at the $N_{D}{ }^{*}$ corresponding to the global minimum cost. As a result, $D / F$ decreases by $5 \%$ and $P_{R}$ increases by $16 \%$. The separation cost only reduces by $1 \%$ because the equipment cost is dominating at $83 \%$ of the total cost. Because the minimum cost $N_{D}{ }^{*}$ was already accessible at 50 psi per column, increasing the pressure limit to 75 psi per column does not change the value of $N_{D}{ }^{*}$ for minimum cost. Only the aspect ratio of the column increases ( $L_{c}$ increases, $I D$ decreases). This change can be explained by the left-hand side of Eq. 30a.

As expected from Fig. 11, decreasing the dead volume from $1.9 \% \mathrm{CV}$ in the base case to zero has little effect on $D / F$ or $P_{R}$, while increasing the dead volume to $10 \% \mathrm{CV}$ increases $D / F$ by $5 \%$ and decreases $P_{R}$ by $16 \%$.

The bed void fraction can only be varied from 0.3 to 0.4 , and does not directly affect $D / F$ (Eq. 19) or $P_{R}$ for diffusion controlled systems (Eq. 27b), as expected. However, it indirectly affects $P_{R}$ because larger $\varepsilon_{b}$ values result in longer columns for the same pressure drop, Eq. (22), which allows for a smaller $N_{D}{ }^{*}$ value, which results in slightly higher $P_{R}(2 \%)$. However, the overall impact on $D / F$ or $P_{R}$ is relatively small for this system. 

not have much of an effect on $D / F$ or $P_{R}$. However, decreasing $\varepsilon_{\mathrm{p}}$ to 0.5 reduces both the

859 dimensionless diffusion rate, $N_{D}{ }^{*}$, and the $\delta$ 's, resulting in increasing $D / F$ by $10 \%$, Eq. (25), and 860 decreasing $P_{R}$ by $65 \%$, Eq. (27b). (25), but increases $P_{R}$ by $11 \%$, Eq. (27b). Decreasing the diffusivity ratio to 1 increases $D / F$ by

$8638 \%$ and decreases $P_{R}$ by $34 \%$, as expected from the trends shown in Fig. 12.

865 from 1.3 to 7.7 , which reduces $D / F$ by $23 \%$ and increases $P_{R}$ by $676 \%$, Eq. (25) and Eq. (27b),

866 respectively. Further reduction of the size-exclusion coefficient of insulin to 0.01 increases the

867 selectivity to $\sim 23$, does not further reduce $D / F$ and further increases $P_{R}$ by $9 \%$.

868 Low pressure sorbents do not typically have radii much smaller than 25 microns, because 869 smaller particles will require a packing length much shorter than $10 \mathrm{~cm}$, as expected from Fig.

8708 b. Decreasing the particle radius from 54 to $25 \mu \mathrm{m}$ reduces $D / F$ by $9 \%$ and increases $P_{R}$ by

$871110 \%$, Eqs. $(13,15)$. However, $L_{c}$ is impractically small because of the limited pressure drop of

8721.5 psi per column. Increasing $R_{p}$ to $100 \mu \mathrm{m}$ increases $D / F$ by $16 \%$ and decreases $P_{R}$ by $53 \%$,

873 Eq. (13) and Eq. (15), respectively.

874 The result of combining all the hypothetical changes in the material properties and 875 equipment parameters is shown in the last row of Table 6 . The $D / F$ approaches that of an ideal 876 system, as a result of the high selectivity and zero $D V$. The $P_{R}$ increases by 66 fold mainly

877 because of the synergistic effects of reducing particle size, increasing selectivity, and increasing 878 the pressure limit. The total cost approaches the lowest possible total cost for the given feed 879 concentration, because the sorbent cost becomes negligible and the solvent cost approaches that 
880 of an ideal system. Further decrease in unit solvent cost is only possible if the feed concentration

881 can be increased. Increasing feed concentration would also reduce the unit equipment cost

882 because the production rate is increased using the same equipment. The results in Table 6

883 indicate that the factors with the largest impact on solvent and sorbent costs are particle size,

884 selectivity, and pressure limit.

885 In this example, equipment cost is dominating (81-90\%) because of the small production

886 scale $(5,000 \mathrm{~kg} /$ year $)$. Optimization of the material properties and equipment parameters has

887 limited impact on the total separation cost (8\%). However, for other applications with much

888 larger production scales, optimization of the material properties and equipment parameters can

889 significantly reduce the total separation cost.

\section{$890 \quad 4.0$ Conclusions}

891 By solving the SWD equations with dimensionless groups, analytical expressions for 892 solvent consumption $(D / F)$ and sorbent productivity $\left(P_{R}\right)$ were obtained for SEC-SMBs. Solvent

893 consumption and sorbent productivity are clearly related to the material properties, equipment

894 parameters, and operating parameters. The sensitivity of solvent cost, sorbent cost, and total cost

895 with respect to the material, equipment, and operating parameters was also elucidated.

896 The results of the parametric studies show that for diffusion controlled systems, one can

897 reduce $D / F$ and increase $P_{R}$ by increasing selectivity $(\alpha)$, diffusivity ratio $(\gamma)$, or $\gamma / \alpha$. Decreasing

898 retention factors $\left(\delta_{i}\right)$ reduces $D / F$, but also decreases $P_{R}$. The phase ratio $(\phi)$ does not affect $D / F$

899 or $P_{R}$. When the yield specification is larger than $99 \%, D / F$ significantly increases and $P_{R}$

900 significantly decreases. The key dimensionless group governing the operating parameters is $N_{D}{ }^{*}$.

901 There is a minimum value $(N D, \min *)$ required for the operation to be feasible. Increasing $N_{D}{ }^{*}$ 
902 will always reduce $D / F$, but the $P_{R}$ peaks at about twice $N D, \min *$, after which $P_{R}$ decreases

903 with increasing $N_{D}{ }^{*}$. The column configuration $\left(N^{j}\right)$ does not significantly affect $D / F$, but can

904 significantly increase the peak $P_{R}$. Dead volume $(D V)$ should be reduced to less than $2 \%$ of the

905 total packing volume to minimize its impact on $D / F$ and $P_{R}$. The competing effects of solvent

906 consumption and $P_{R}$ result in a cost minimum because solvent cost always decreases with $N_{D}{ }^{*}$

907 and sorbent cost increases when ${N_{D}}^{*}>N D, \max P R \star$. However, the cost minimum may not be

908 achievable if the system has a pressure limit. The combination of $\left(\Delta P R_{p}{ }^{4} / L_{c}{ }^{2}\right)$ which allows

909 access to the cost minimum can be calculated from $N D$, $\max P R *$, material properties (other

910 than $R_{p}$ ), yields, column configuration, and viscosity, Eq. (30a). The corresponding $N_{\Delta P, \text { diff }}$ for

911 maximum $P_{R}$ is about 1 for the example system. Thus, the diffusion time, step time, and

912 pressure-limited convection time are approximately equal at the maximum $P_{R}$.

913 For dispersion controlled systems, $D / F$ can be reduced and $P_{R}$ can be increased by

914 increasing $\alpha$ and increasing retention factors. The dependence of $D / F$ on retention factors is

915 opposite to that of diffusion controlled systems. Increasing phase ratio does not significantly

916 reduce $D / F$, but does increase $P_{R}$. The effects of yield specification on $D / F$ and $P_{R}$ are similar to

917 those for diffusion controlled systems. The key dimensionless group controlling the operating

918 parameters is $P_{e b}{ }^{*}$. The effects of $P_{e b}{ }^{*}$ on $D / F$ and $P_{R}$ are also similar to those of $N_{D}{ }^{*}$ for diffusion

919 controlled systems. The column configuration and $D V$ have similar effects on $D / F$ and $P_{R}$ as

920 those of diffusion controlled systems. The value of $\left(\Delta P_{\max } R_{p}{ }^{2} / L_{c}\right)$ can be found at the value of

$921 P e b \star$ for minimum cost from Eq. (39), such that the minimum cost design satisfies the pressure

922 limit. The value of $P e b, \max P R \star$ can be calculated from yields, material properties, and column 
923 configuration. The maximum $P_{R}$ occurs when the axial dispersion time is about 10 times the step

924 time and about 50 times the pressure-limited convection time.

925 The recast SWD solutions of $D / F$ and productivity can be used to optimize SMB designs.

926 This method was demonstrated using insulin purification as an example and the optimal SMB

927 design was compared to the industrial batch process. Optimization of 11 parameters (column

928 length, column configuration, operating pressure, and operating parameters) reduces the solvent

929 consumption from batch SEC by $66 \%$, increases the sorbent productivity by 34 times, and

930 reduces the total cost by $62 \%$. If the material properties, pressure limit, and dead volume are

931 allowed to change, solvent consumption can be further reduced by $28 \%$ and productivity can be

932 further increased by 67 times. Since the equipment cost dominates ( $81 \%$ of total cost) at this

933 relatively small production scale, optimization of the material properties and pressure limit only

934 reduces the separation cost by $8 \%$, Table 6 . Among the material properties, selectivity and

935 particle size have the largest impact on solvent consumption and sorbent productivity. For

936 applications with large production scales, the unit equipment cost is expected to be a small

937 fraction of the total cost, and this general optimization method is expected to significantly reduce

938 solvent consumption, increase sorbent productivity, and reduce separation cost.

\section{$939 \quad 5.0$ Acknowledgments}

940 This work was supported by the National Science Foundation (CBET 1403854) and the

941 Hugh and Edna Donnan Dissertation Fellowship (Purdue University).

\section{$942 \quad 6.0$ References}

943 [1] R. Guo, Z. Shi, X. Wang, A. Dong, J. Zhang, Separation and quantification of dead species in styrene RAFT polymerization by gradient polymer elution chromatography, Polym. Chem. 3 (2012) 1314. doi:10.1039/c2py20102j. 
946

947

948

949

950

951

952

953

954

955

956

957

958

959

960

961

962

963

964

965

966

967

968

969

970

971

972

973

974

975

976

977

978

979

[2] E. Uliyanchenko, S. van der Wal, P.J. Schoenmakers, Challenges in polymer analysis by liquid chromatography, Polym. Chem. 3 (2012) 2313. doi:10.1039/c2py20274c.

[3] E.P. Kroeff, R.A. Owens, E.L. Campbell, R.D. Johnson, H.I. Marks, Production scale purification of biosynthetic human insulin by reversed-phase high-performance liquid chromatography., J. Chromatogr. 461 (1989) 45-61. doi:10.1016/S0021-9673(00)942742 .

[4] D. Broughton, C.G. Gerhold, Continuous sorption process employing fixed bed of sorbent and moving inlets and outlets, 2,985,589, 1961.

[5] D. Broughton, Molex: Case history of a process, Chem. Eng. Prog. 64 (1968) 60-65.

[6] D. Broughton, Production-scale adsorptive separations of liquid mixtures by simulated moving-bed technology, Sep. Sci. Technol. (1984) 37-41. http://www.tandfonline.com/doi/abs/10.1080/01496398408068590 (accessed July 29, 2013).

[7] L. Pais, J. Loureiro, A. Rodrigues, Separation of 1, 1'-bi-2-naphthol enantiomers by continuous chromatography in simulated moving bed, Chem. Eng. Sci. 52 (1997). http://www.sciencedirect.com/science/article/pii/S0009250996003983 (accessed July 29, 2013).

[8] Y. Xie, S. Mun, J. Kim, N.-H.L. Wang, Standing wave design and experimental validation of a tandem simulated moving bed process for insulin purification., Biotechnol. Prog. 18 (2002) 1332-44. doi:10.1021/bp025547r.

[9] S. Mun, Y. Xie, J.-H. Kim, N.-H.L. Wang, Optimal Design of a Size-Exclusion Tandem Simulated Moving Bed for Insulin Purification, Ind. Eng. Chem. Res. 42 (2003) 19771993. doi:10.1021/ie020680+.

[10] J. Houwing, H.A.H. Billiet, L.A.M. van der Wielen, Mass-transfer effects during separation of proteins in SMB by size exclusion, AIChE J. 49 (2003) 1158-1167. doi:10.1002/aic.690490509.

[11] A. Geisser, T. Hendrich, G. Boehm, B. Stahl, Separation of lactose from human milk oligosaccharides with simulated moving bed chromatography., J. Chromatogr. A. 1092 (2005) 17-23. doi:10.1016/j.chroma.2005.03.061.

[12] M.-T. Liang, R.-C. Liang, Fractionation of polyethylene glycol particles by simulated moving bed with size-exclusion chromatography., J. Chromatogr. A. 1229 (2012) 107-12. doi:10.1016/j.chroma.2011.12.094.

[13] G. Biressi, O. Ludemann-Hombourger, M. Mazzotti, R.M. Nicoud, M. Morbidelli, Design and optimisation of a simulated moving bed unit: role of deviations from equilibrium 
1004 1005 1006 1007

1008 1009 1010

1011 1012 1013 theory., J. Chromatogr. A. 876 (2000) 3-15.

http://www.ncbi.nlm.nih.gov/pubmed/10823498.

[14] Z. Ma, N.-H.L. Wang, Standing wave analysis of SMB chromatography: Linear systems, AIChE J. 43 (1997) 2488-2508. doi:10.1002/aic.690431012.

[15] B.J. Hritzko, Y. Xie, R.J. Wooley, N.-H.L. Wang, Standing-wave design of tandem SMB for linear multicomponent systems, AIChE J. 48 (2002) 2769-2787. doi:10.1002/aic.690481207.

[16] T. Mallmann, B. Burris, Z. Ma, N. Wang, Standing wave design of nonlinear SMB systems for fructose purification, AIChE J. 44 (1998). http://onlinelibrary.wiley.com/doi/10.1002/aic.690441206/abstract (accessed July 28, 2013).

[17] Y. Xie, C.A. Farrenburg, C.Y. Chin, S. Mun, N.-H.L. Wang, Design of SMB for a nonlinear amino acid system with mass-transfer effects, AIChE J. 49 (2003) 2850-2863. doi:10.1002/aic.690491117.

[18] K. Lee, S. Mun, F. Cauley, G. Cox, N.-H.L. Wang, Optimal standing-wave design of nonlinear simulated moving bed systems for enantioseparation, Ind. Eng. Chem. Res. (2006) 739-752. http://pubs.acs.org/doi/abs/10.1021/ie0504248 (accessed July 28, 2013).

[19] K. Lee, C. Chin, Y. Xie, G. Cox, N.-H.L. Wang, Standing-wave design of a simulated moving bed under a pressure limit for enantioseparation of phenylpropanolamine, Ind. Eng. Chem. Res. (2005) 3249-3267. http://pubs.acs.org/doi/abs/10.1021/ie049413p (accessed July 28, 2013).

[20] G. Paredes, M. Mazzotti, Optimization of simulated moving bed and column chromatography for a plasmid DNA purification step and for a chiral separation., J. Chromatogr. A. 1142 (2007) 56-68. doi:10.1016/j.chroma.2006.12.009.

[21] F.G. Cauley, S.F. Cauley, K.B. Lee, Y. Xie, N.-H.L. Wang, Standing Wave Annealing Technique: For the design and optimization of nonlinear simulated moving bed systems with significant mass-transfer effects, Ind. Eng. Chem. Res. 45 (2006) 8697-8712. doi:10.1021/ie060300a.

[22] F.G. Cauley, Y. Xie, N.-H.L. Wang, Optimization of SMB systems with linear adsorption isotherms by the Standing Wave Annealing Technique, Ind. Eng. Chem. Res. 43 (2004) 7588-7599. doi:10.1021/ie049842n.

[23] F.G. Cauley, S.F. Cauley, N.-H.L. Wang, Standing wave optimization of SMB using a hybrid simulated annealing and genetic algorithm (SAGA), Adsorption. 14 (2008) 665678. doi:10.1007/s10450-008-9119-8. 
1014 [24] K. Lee, R. Kasat, G. Cox, N. Wang, Simulated moving bed multiobjective optimization using standing wave design and genetic algorithm, AIChE J. 54 (2008). doi:10.1002/aic.

1016

1017

1018

1019

[25] L. Ling, P.L. Chung, A. Youker, D.C. Stepinski, G.F. Vandegrift, N.H.L. Wang, Capture chromatography for Mo-99 recovery from uranyl sulfate solutions: Minimum-columnvolume design method, J. Chromatogr. A. 1309 (2013) 1-14. doi:10.1016/j.chroma.2013.08.023.

1020 [26] S. Ergun, Fluid flow through packed columns, Chem. Eng. Prog. 48 (1952) 89-94.

1021 [27] M.M. Awad, Hagen number versus bejan number, Therm. Sci. 17 (2013) 1245-1250.

1022 doi:10.2298/TSCI1304245A.

1023 [28] S.F. Chung, C.Y. Wen, Longitudinal dispersion of liquid flowing through fixed and 1024 fluidized beds, AIChE J. 14 (1968) 857-866. doi:10.1002/aic.690140608.

1025

1026

1027

[29] D.A. Horneman, M. Wolbers, M. Zomerdijk, M. Ottens, J.T.F. Keurentjes, L.A.M. van der Wielen, Surfactant-aided size exclusion chromatography., J. Chromatogr. B. Analyt. Technol. Biomed. Life Sci. 807 (2004) 39-45. doi:10.1016/j.jchromb.2004.01.061.

1028

1029 
1030 Appendices

1031 Appendix A-Additional Equations and Cost Optimization

1032

1033

\section{Appendix A1 - Equations Relating Bij to Yields and Zone Velocities}

1034

The equations for the $\beta$ terms are given by Eq. (A1.1), which were derived from

1035 equations presented in Hritzko et al. [15].

1036

$\beta 2 I=\ln Y 21-Y 2 u O I I I u O I V-11-u O I I u O I$

1037

(A1.1a)

1038

в 1II=InuOIиOII - 11-Y1Y11- иOIVuOIII-1

1039

(A1.1b)

1040

$\beta 2 I I I=\ln 1-u O I V u O I I I 1-Y 2 Y 2 u O I u O I I-1+1$

1041

(A1.1c)

1042

$\beta 1 I V=\ln Y 11-Y 11-u$ OІІ ОІиОІІІиОIV-1

1043

(A1.1d)

1044 Appendix A2 - Expression of $\Gamma j U$ sing the Chung and Wen Correlation for Low Re The Chung and Wen correlation for low $\operatorname{Re}(\operatorname{Re}<10)($ Eq. 34) was used to evaluate the

1046 ratios of axial dispersion coefficients $\left(\Gamma^{j}\right)$ from Eq. (9). The resulting equations are rearranged to 1047 solve for $\Gamma^{j}$, Eq. (A2.1).

$1048 \Gamma I=1+\phi \delta 21+\phi \delta 1-\phi \delta 2-\delta 1 P e b \star \beta 1 I V N I V+\beta 2 I N I$ 


\section{Systems}

1056

The Peclet number for maximum productivity, $P e b, \max P R *$, at a constant port velocity

1057 can be found by taking the partial derivative of Eq. (37b) with respect to $P_{e b}{ }^{*}$ and setting the

1058 resulting equation equal to zero. The expression that $P e b, \max P R \star$ must satisfy is given by Eq.

1059 (A3.1).

1060

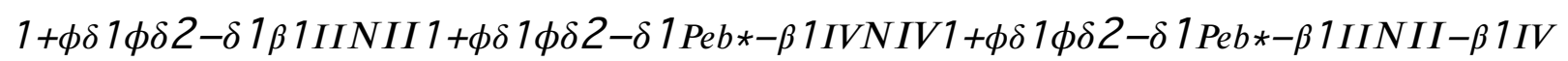

1065 At a large $P_{e b}{ }^{*}$, or a large number of columns in all zones, Eq. (A3.1a) simplifies to Eq. (A3.1b).

$1066 P e b, \max P R *=2 \beta 1 I I N I I+21+\phi \delta 21+\phi \delta 1 \beta 2 I I I N I I I$ 


\section{Appendix A4 - Cost Functions}

1069 The total cost (TC) is defined as the sum of the Equipment Cost (EC), Resin Cost (RC), 1070 and Solvent Cost (SC), Eq. (A4.1).

$1071 T C=E C+R C+S C$

1072 The purchase cost of the equipment is assumed to be $\$ 400,000$ plus $\$ 10,000$ per column 1073 with a depreciation time of seven years and 4\% downtime for a production scale of 5,000 kg 1074 insulin per year. The unit equipment cost is given by Eq. (A4.2)

1075 EC $\$$ kginsulin=400,000\$+10,000\$columnNcolumns 7 years $0.965,000$ kginsulinyear

1077 The purchase cost for the sorbent is assumed to be $\$ 150$ per liter. The usable life of the 1078 sorbent is assumed to four years. The diameter of the columns is determined by the production 1079 rate and the zone velocities. The unit sorbent cost is given by Eq. (A4.3).

$1080 \quad$ RC \$kginsulin=Bed

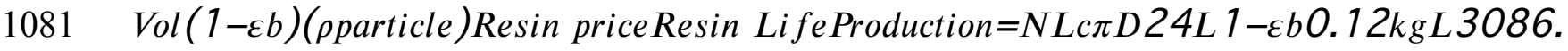

$10825 \$ \mathrm{~kg} 4$ years 5,000 kginsulinyear (A4.3)

1083 The purchase price of solvent is assumed to be $\$ 0.10$ per liter. Assuming no recycle of 1084 the recycle, the unit solvent cost is given by Eq. (A4.4).

1085 SC \$kginsulin=1+DFSolvent priceFeed Conc.Yield=1+DF0.1\$/L 1,00Oginsulinkginsu

1086 linFeed conc. ginsulinLYield

Batch SEC equipment cost is assumed to be $\$ 100,000$ per unit of 12 columns with the 1088 same depreciation rate, resin life, and utilization factor as that of the SEC-SMB. 


\section{Appendix A5 - Cost Optimization Algorithm}

1090 The algorithm that was used to determine the optimum column configuration, column

1091 length, zone velocities, and port velocity for minimum cost is shown in Fig. A5.1. Material

1092 properties, yields, dead volume, pressure limit, feed concentration, packing density, fluid

1093 viscosity, and production scale were held constant. The total number of columns was varied from

1094 eight to twelve and each zone was constrained to have at least two columns. The number of

1095 columns in Zone IV was calculated from the column balance of the other zones and the total

1096 number of columns. $N_{D}{ }^{*}$ ranged from 0.02 to 100 and $P_{e b}{ }^{*}$ ranged from 5 to 1,000 . The initial

1097 values of the variables were sent to the SSWD algorithm, Fig. A5.1b. This algorithm used an

1098 initial guess for $\beta_{i}^{j}$ and $\Gamma^{j}$ and then evaluated $u_{0}^{j} / \nu$. The SSWD algorithm then calculated $\beta_{i}^{j}$ and

$1099 \Gamma^{j}$ from $u_{0}^{j} / v$ using Eq. (A1.1) and Eq. (A2.1), respectively. If the calculated values were not

1100 within a tolerance of 0.001 of the guessed values, then the $u_{0}^{j} / v$ calculations were repeated with

1101 the new values of $\beta_{i}^{j}$ and $\Gamma^{j}$. Once the values were within the tolerance, $L_{c}$ was calculated from

$1102 P_{e b}{ }^{*}$ using Eq. (36b) and $v$ was calculated from $N_{D}{ }^{*}$ using Eq. (6) and Eq. (16). After $v$ was

1103 calculated, the zone velocities could be calculated using Eq. (18). The solvent consumption and

1104 sorbent productivity were then calculated using Eq. (19) and Eq. (20), respectively. The total cost

1105 of the separation was then calculated using Eqs. (A4.1-A4.4). The pressure drop of the system

1106 was calculated from Eq. (22) and compared to the set pressure limit of 1.5 psi per column. If the

1107 calculated pressure exceeded the pressure limit, then the design was not considered feasible.

1108 After the pressure check, the Peclet number was incremented and the process repeated until it

1109 reached 1,000 . Then $N_{D}{ }^{*}$ was incremented and the $P_{e b}{ }^{*}$ cycle was repeated. Once $N_{D}{ }^{*}$ reached a

1110 value of 100, the column configuration would change, and the $N_{D}{ }^{*}$ and $P_{e b}{ }^{*}$ cycles would repeat.

1111 After all the cycles were completed, the minimum cost was found from all the stored values and 
1112 the associated cost, $L_{c}, N^{j}, u_{0}^{j}$, and $v$ were determined. This algorithm was implemented using 1113 MATLAB R2014a on a laptop computer with a total run time of less than one minute.

1114 Appendix B - Parameters from Literature Cases

1115 The expanded sets of parameters are shown in tables for Case 1 (Table B.1), Case 2

1116 (Table B.2), and Case 3 (Table B.3).

1117 


\section{Figure Captions}

1119 Figure 1. Diagram of a four-zone SMB. (a) Step N; (b) step N + 1.

1120

1121

1122

1123

1124

1125

1126

1127

1128

1129

1130

1131

1132

1133

1134

1135

1136

1137

1138

1139

1140

1141

1142

1143

1144

1145

1146

1147

1148

1149

1150

1151

1152

1153

1154

1155

1156

1157

1158

1159

Figure 2. Design overview.

Figure 3. Standing Wave Design mid-step concentration profiles for a binary, non-ideal separation.

Figure 4. Comparison of (a) $D / F$ and (b) productivity for Case 1.

Figure 5. Comparison of (a) $D / F$ and (b) productivity for Case 2.

Figure 6. (a) $D / F$ and (b) $P_{R}$ for three column configurations with varying $N_{D}{ }^{*}$ - diffusion controlled. Vertical line represents the minimum $N_{D}{ }^{*}$ allowed by the pressure limitation (designs to the left of the line are not feasible). $D / F$ and $P_{R}$ curves for $N^{j}=2-3-3-2$ are at $99.9 \%$ yield to match data point from Xie et al.

Figure 7. Comparison of (a) $D / F$ and (b) productivity for literature designs and SSWD of SECSMB at the same $N_{D}{ }^{*}$.

Figure 8. (a) Productivity at varying $R_{p}$ and (b) pressure drop per length ${ }^{2}$ vs. $R_{p}$ at varying yields (fixed $N_{D}{ }^{*}=N D, \max P R * \sim 0.2$ ) - diffusion controlled.

Figure 9. (a) $D / F$ and (b) $P_{R}$ vs. yield at various ${N_{D}}^{*}$ - diffusion controlled, 3-3-3-3 configuration.

Figure 10. (a) $D / F$ and (b) $P_{R}$ vs. selectivity at a fixed $N_{D}{ }^{*}$ and various $\delta_{l}$ - diffusion controlled, 3-3-3-3 configuration. Fixed $N_{D, 1}=6.5$.

Figure 11. (a) $D / F$ and (b) $P_{R}$ vs. $D V$ for various $N_{D, 1}$ - diffusion controlled, 3-3-3-3

configuration.

Figure 12. (a) $D / F$ and (b) $P_{R}$ vs. $\gamma$ at various ${N_{D}}^{*}$ - diffusion controlled, 3-3-3-3 configuration.

Figure 13. (a) $D / F$ and (b) $P_{R}$ vs. $P_{e b}{ }^{*}$ for multiple column configurations at constant port velocity $=1 \mathrm{~cm} / \mathrm{min}$ - dispersion controlled. Vertical line represents the maximum $P_{e b}{ }^{*}$ allowed by the pressure limitation (designs to the right of the line are not feasible).

Figure 14. (a) $D / F$ and (b) $P_{R}$ vs. yield at varying $P_{e b}{ }^{*}$. Constant port velocity $=1 \mathrm{~cm} / \mathrm{min}-$ dispersion controlled.

Figure 15. (a) $D / F$ and (b) $P_{R}$ vs. selectivity at various values for $\delta_{1} . P_{e b}{ }^{I V}$ fixed at 50 and port velocity fixed at $1 \mathrm{~cm} / \mathrm{min}$ - dispersion controlled, 3-3-3-3 configuration.

Figure 16. Estimates of total, resin, and solvent costs vs. $N_{D}{ }^{*}-$ diffusion controlled, 2-2-2-2 configuration. Black star indicates minimum achievable cost at pressure limit. 
1161 Figure 17. (a) Inverse of total cost curve vs. $P_{e b}{ }^{*}$ and $N_{D}{ }^{*}$ for Ring A. Pressure limit is indicated 1162 by the black surface and indicates the minimum $N_{D}{ }^{*}$ at each $P_{e b}{ }^{*}$ for the system to not exceed the 1163 maximum pressure. (b) Contour plot of (a). Black dashed line represents the maximum pressure 1164 limit. Regions below the black line are infeasible.

Figure A5.1. (a) algorithm for optimizing 11 decision variables (column configuration, column 1167 length, zone velocities, and port velocity) for minimum cost and (b) algorithm used by SSWD to 1168 determine column length, $\beta_{i}^{j}, \Gamma^{j}$, zone velocities, and port velocity from given dimensionless 1169 groups. 
(a)

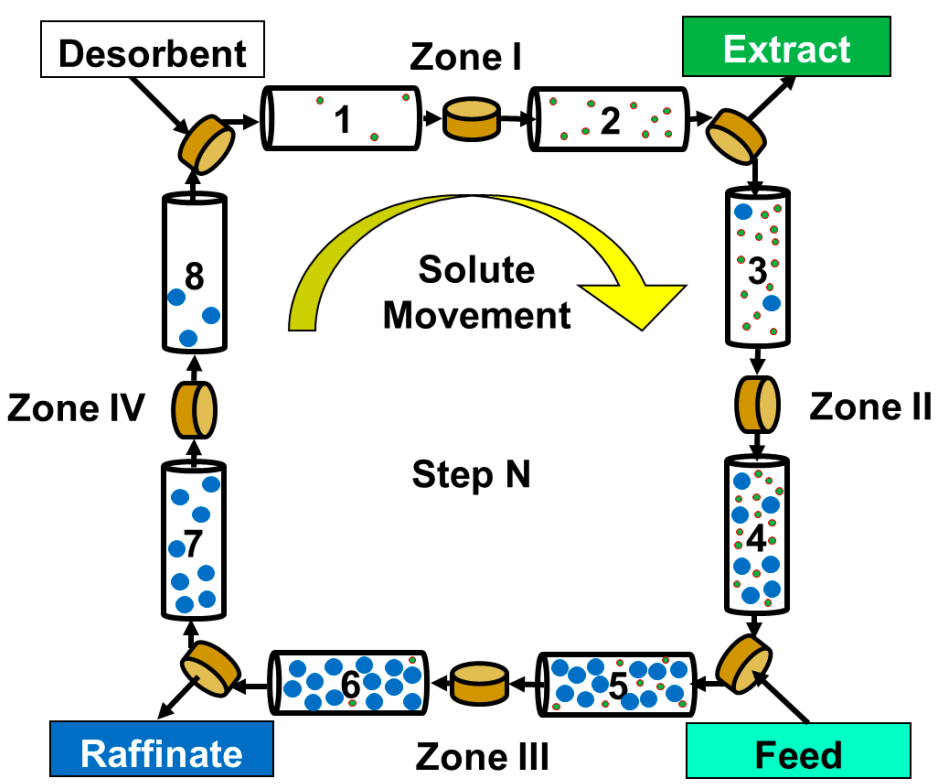

(b)

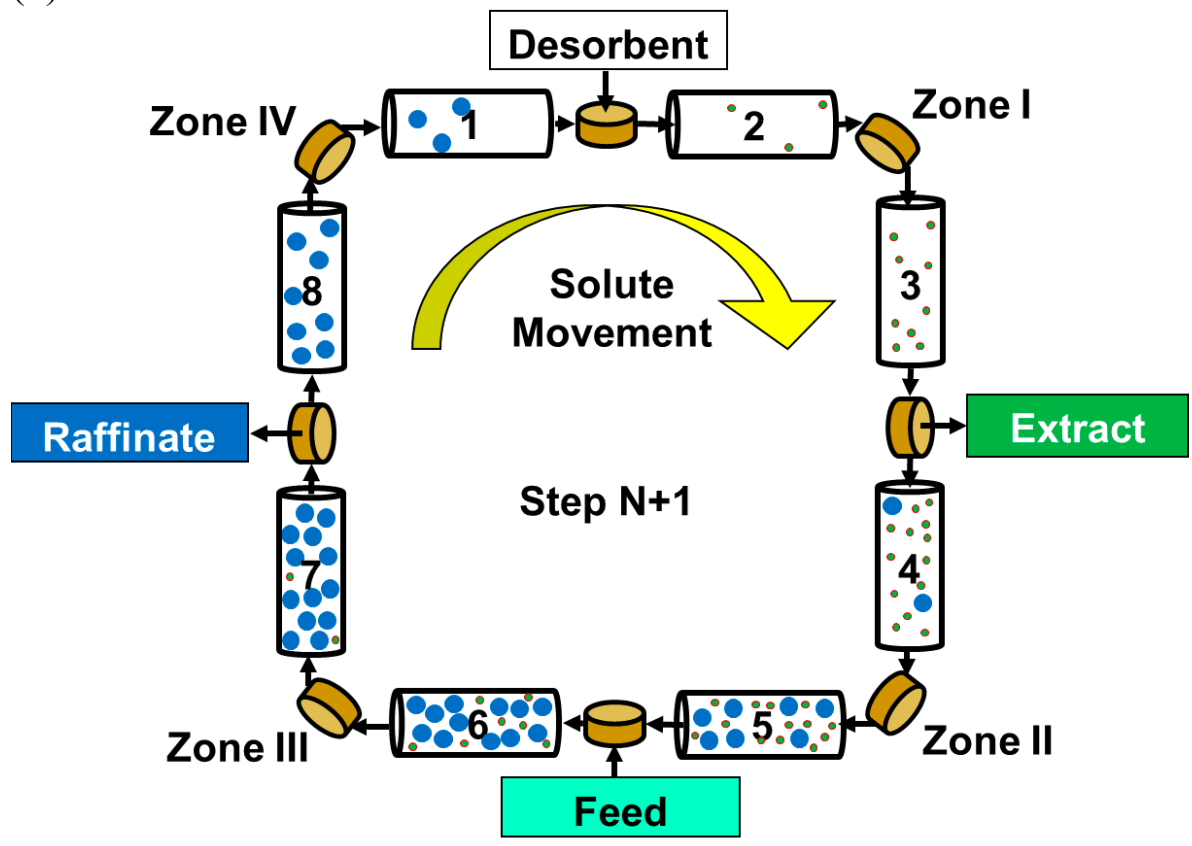




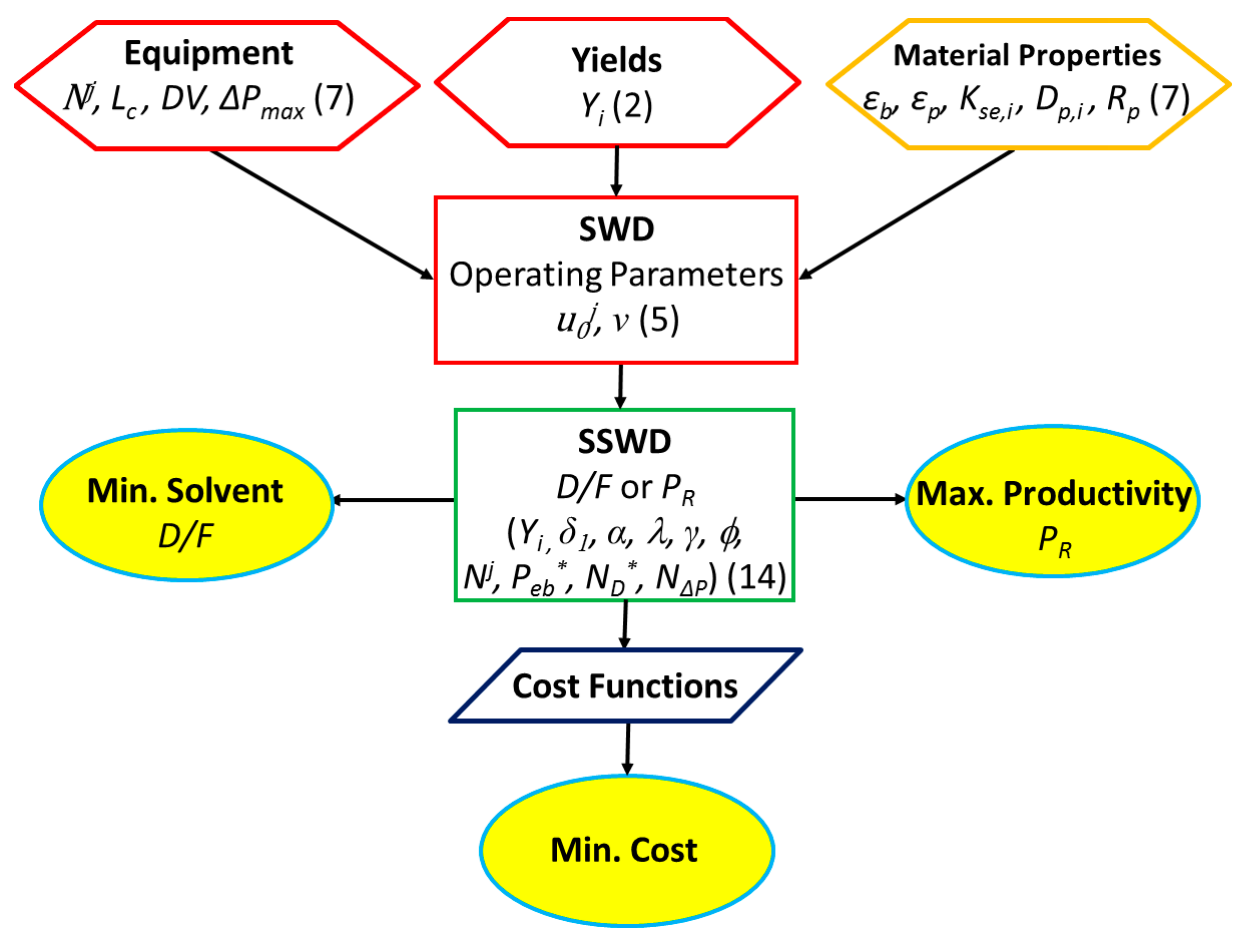




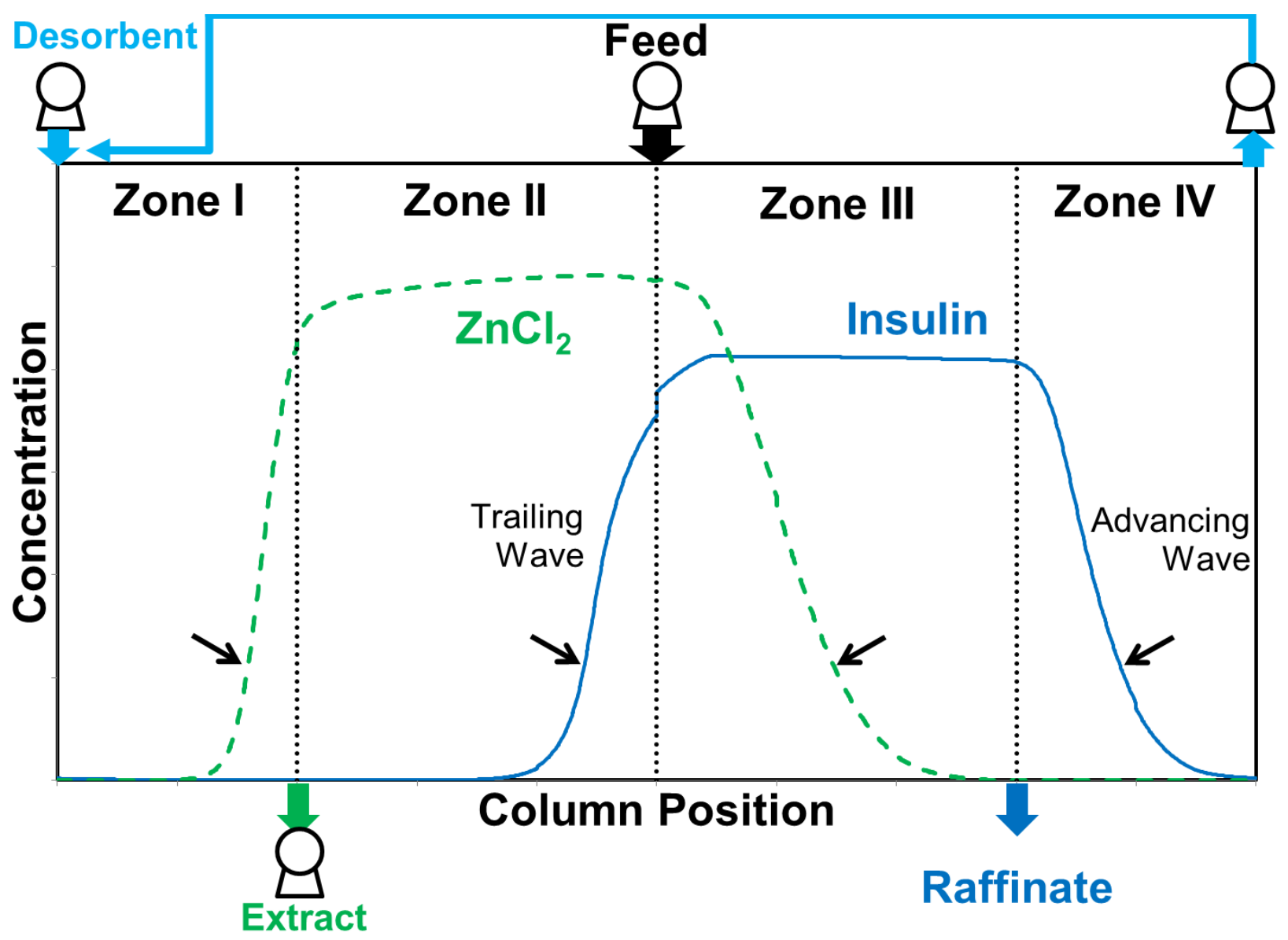


Figure 4

(a)

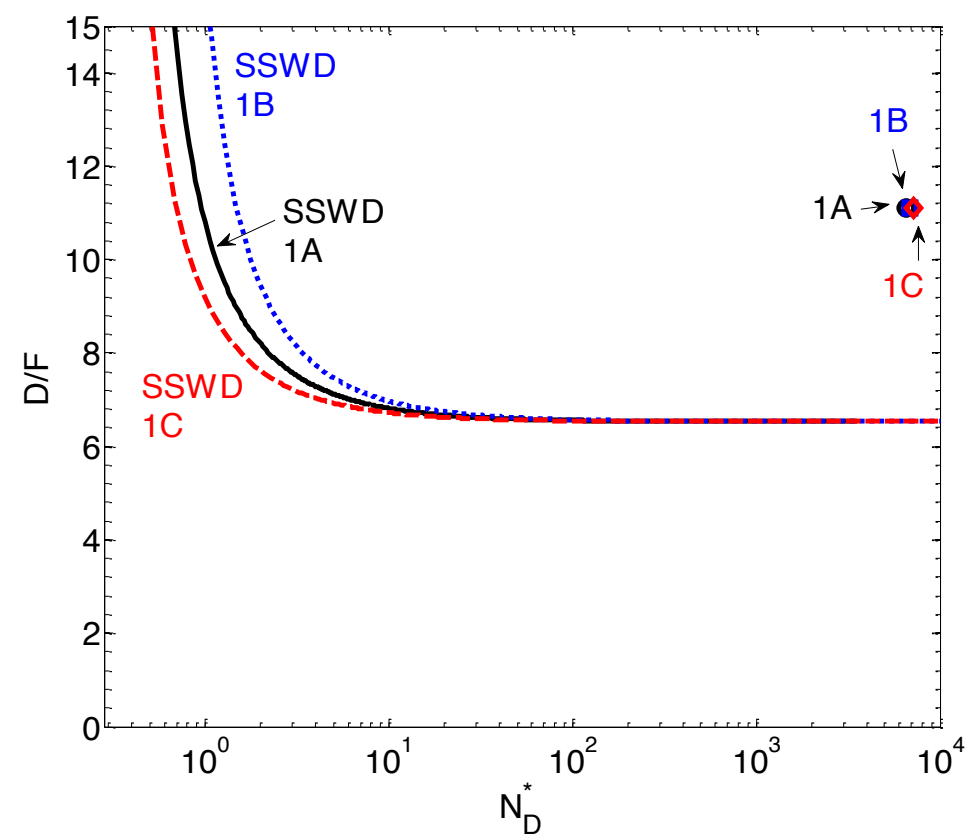

(b)

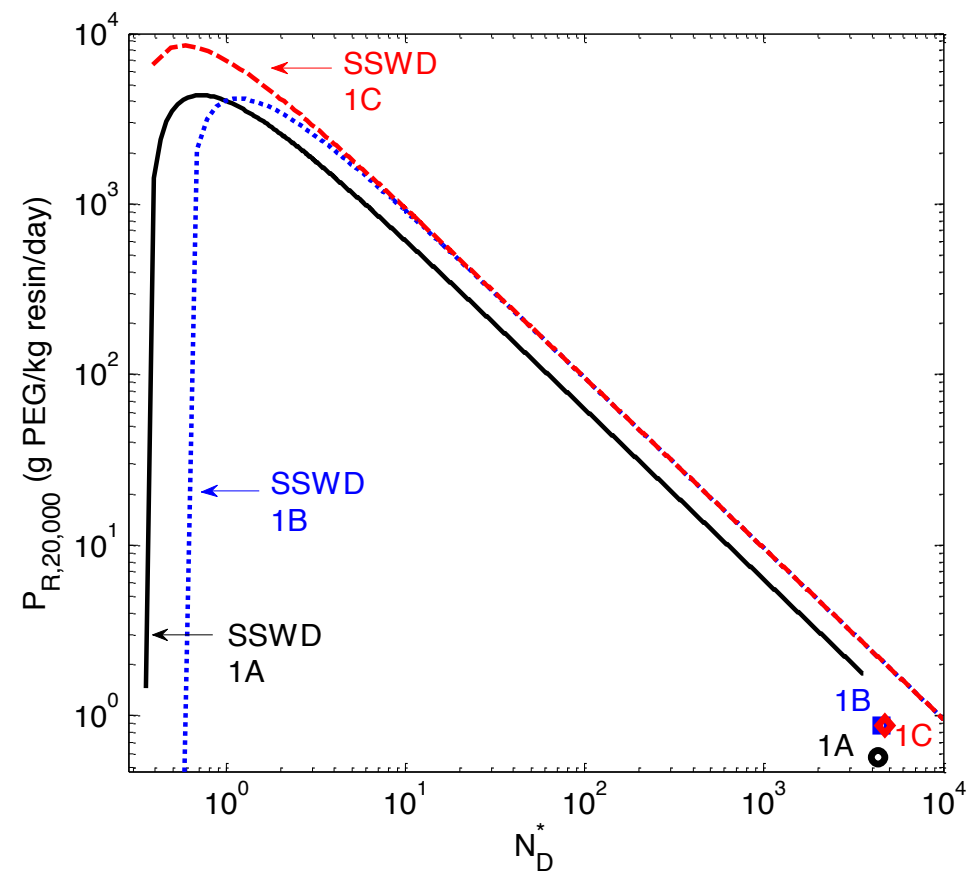


(a)

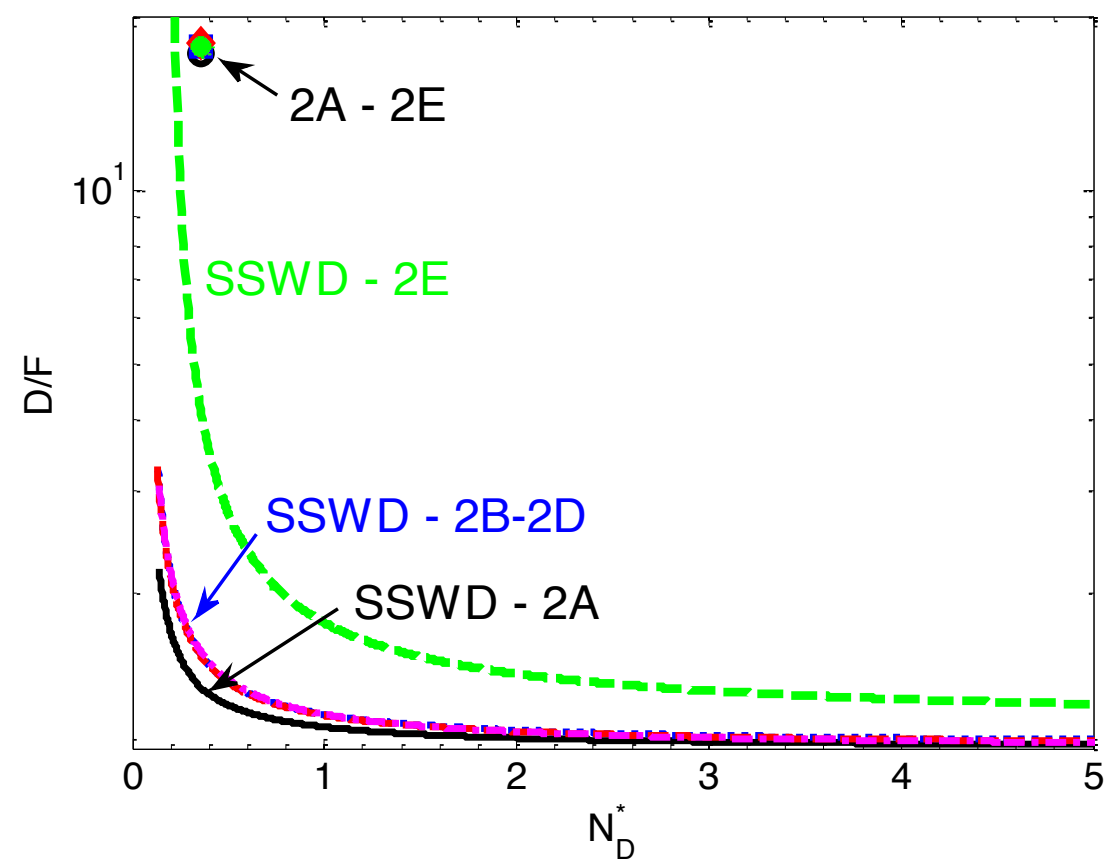

(b)

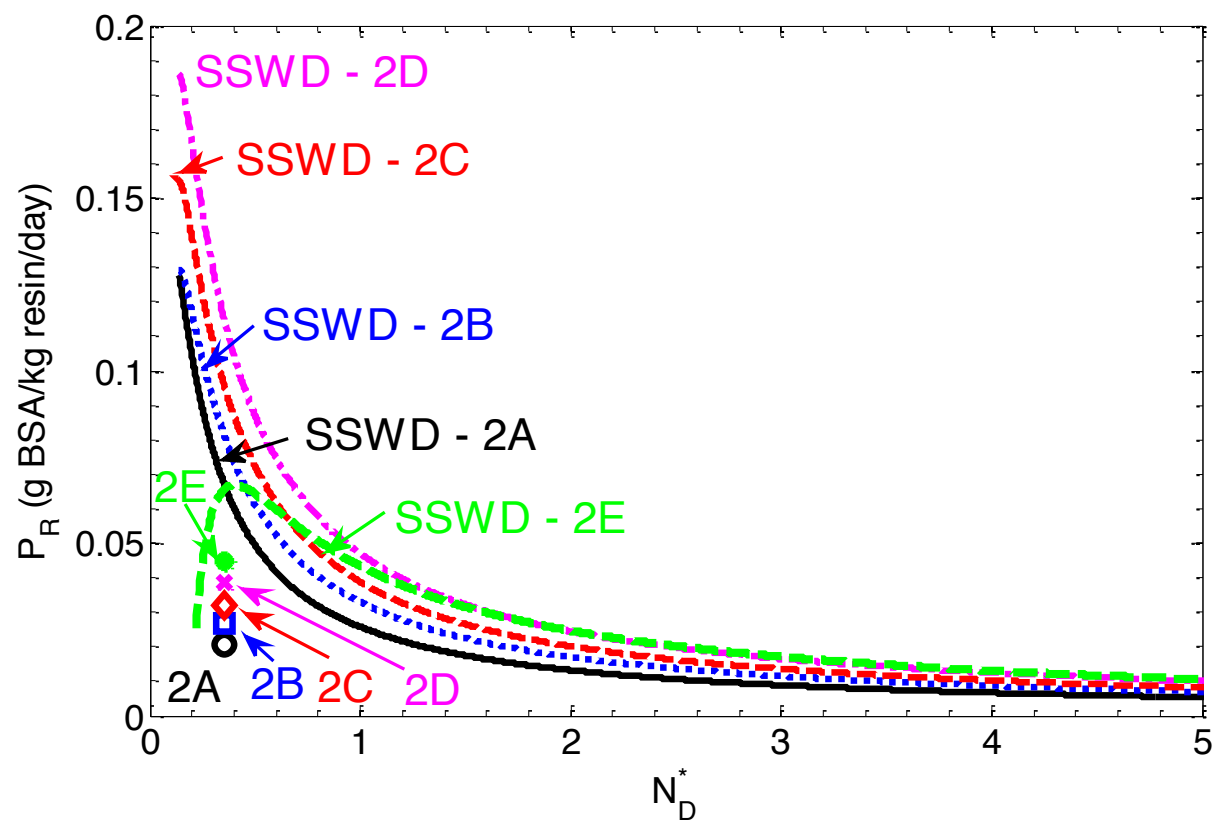


(a)

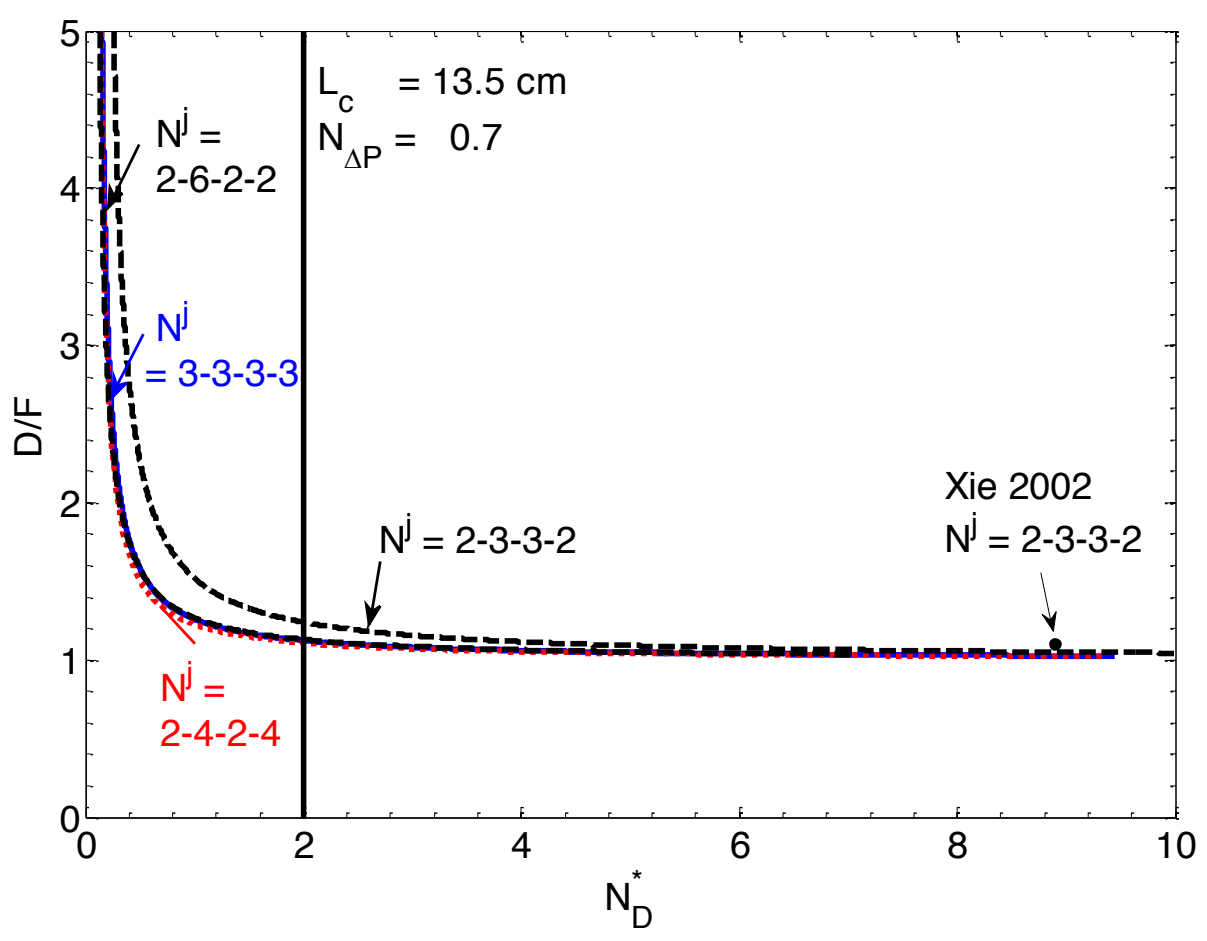

(b)

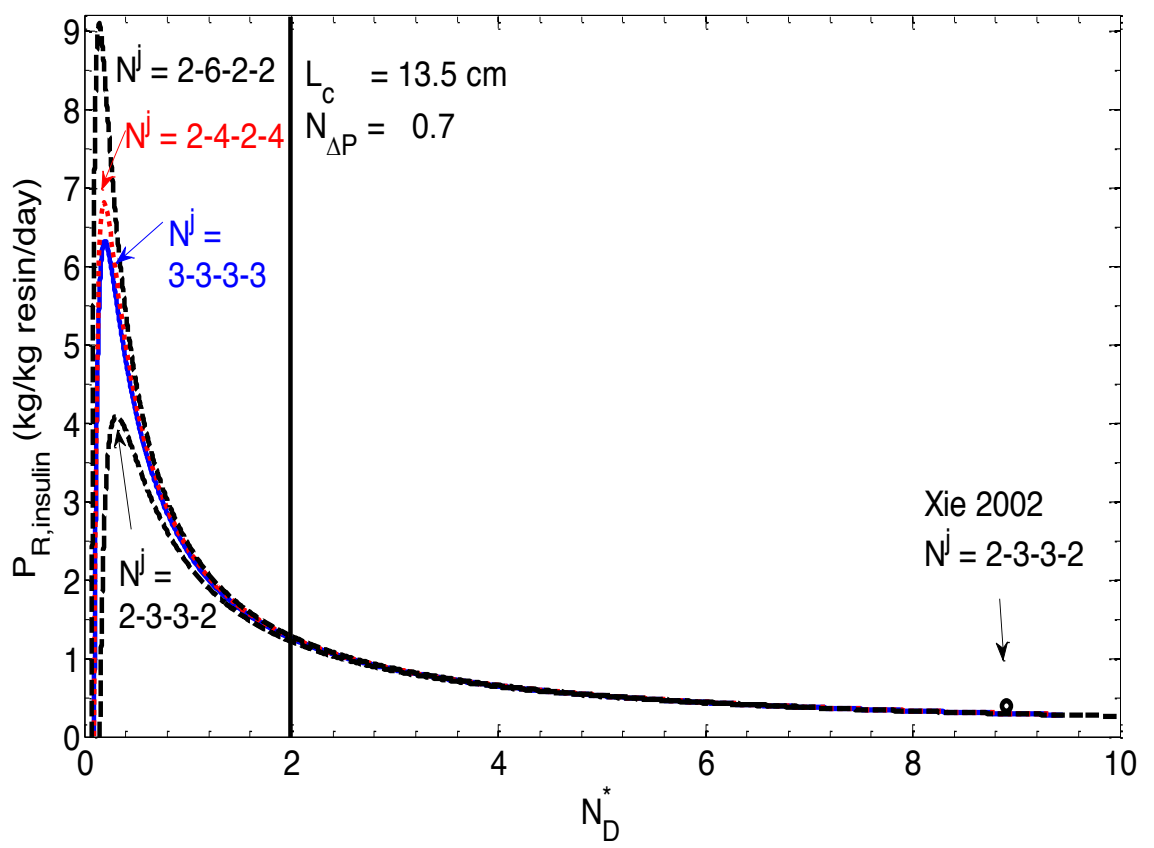


(a)

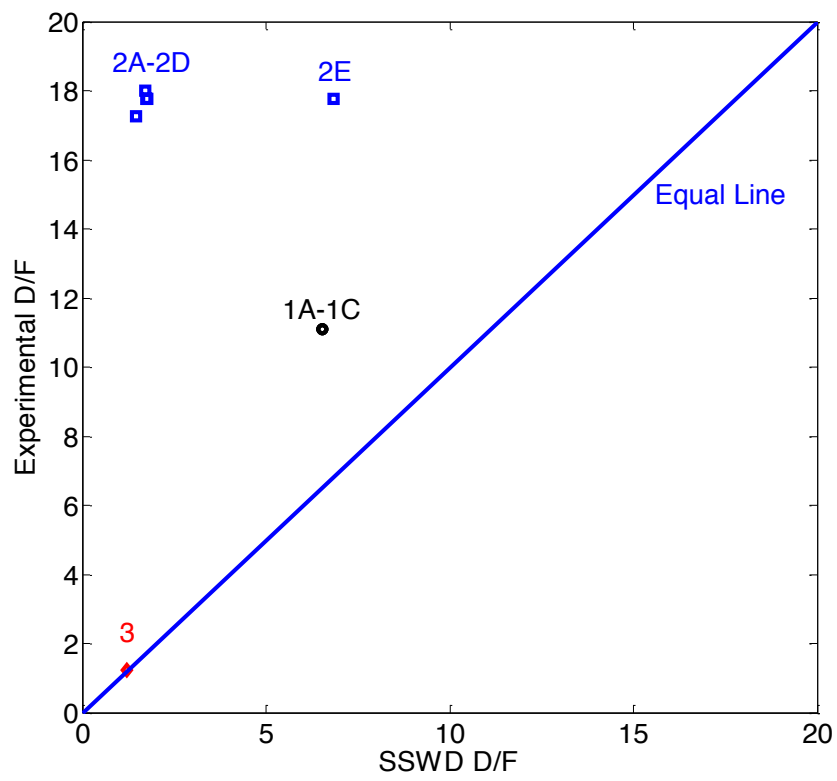

(b)

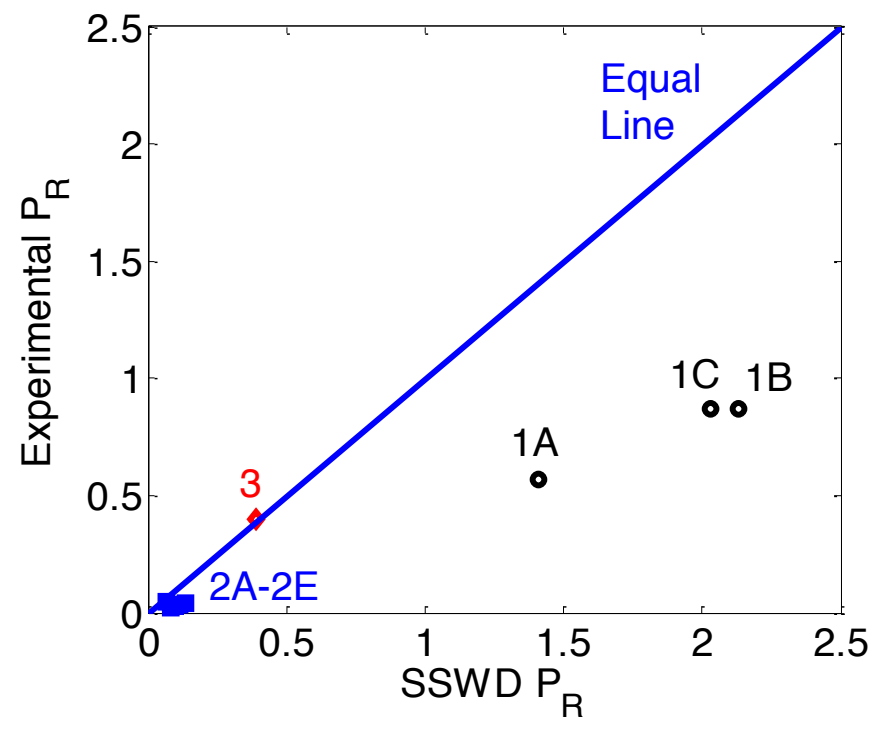


(a)

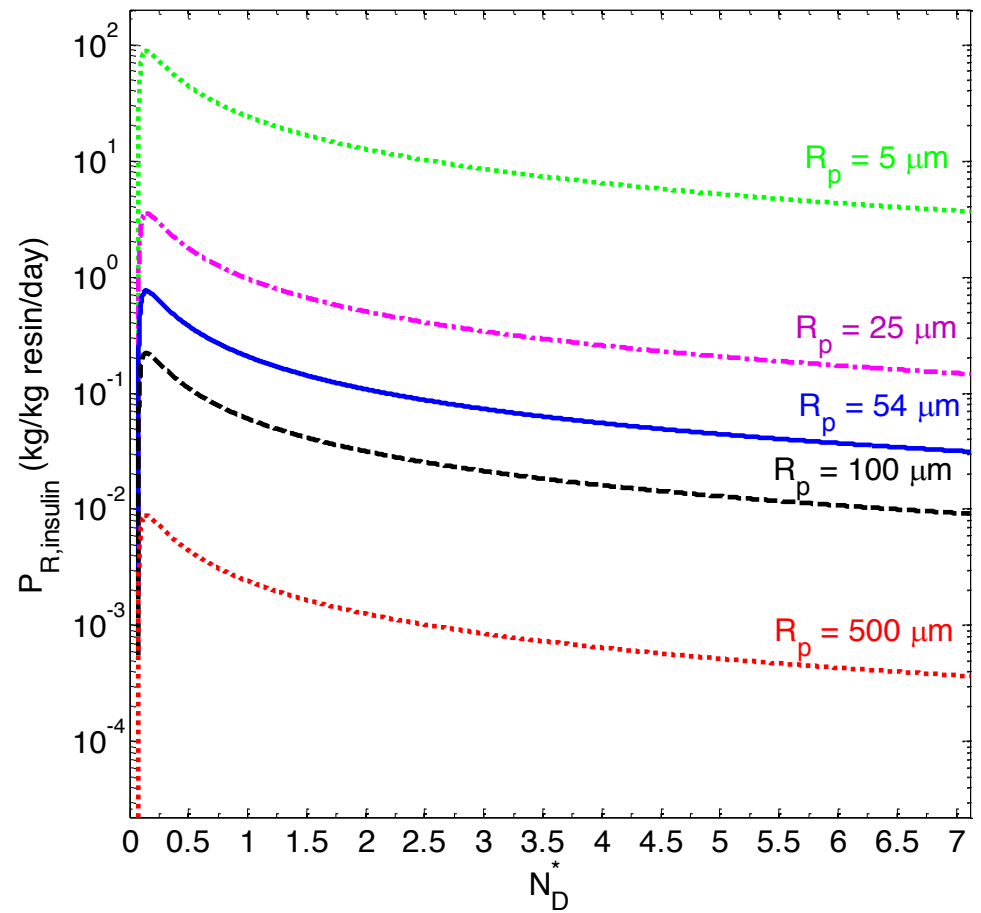

(b)

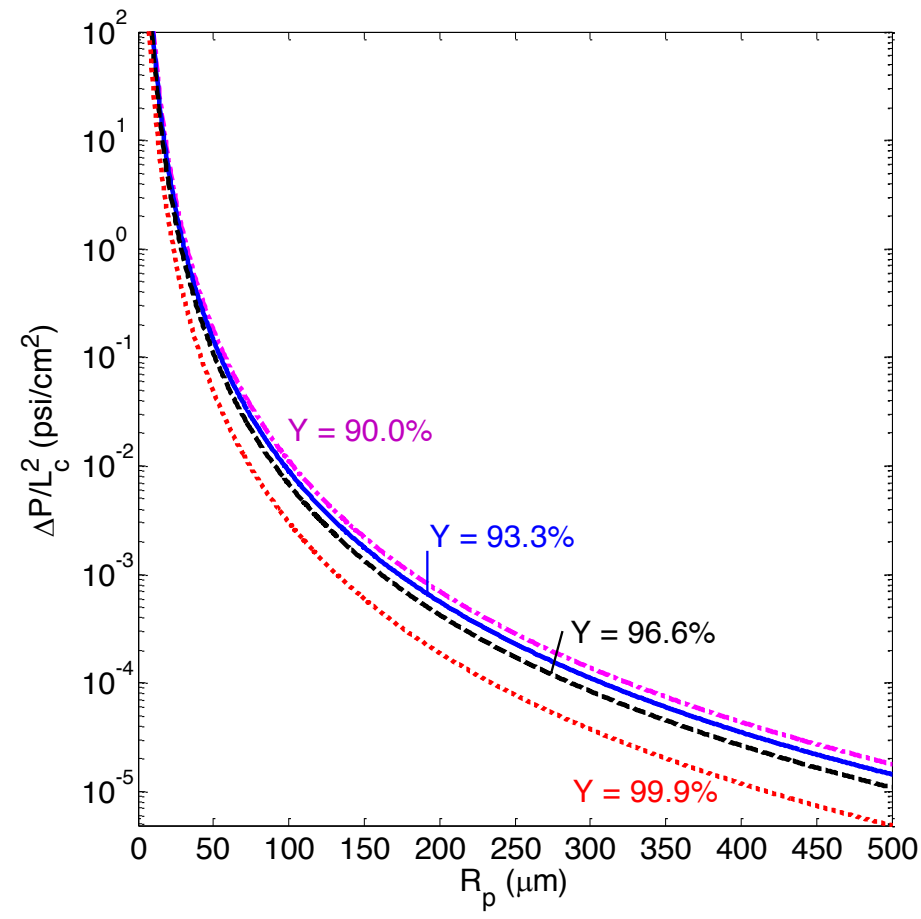


(a)

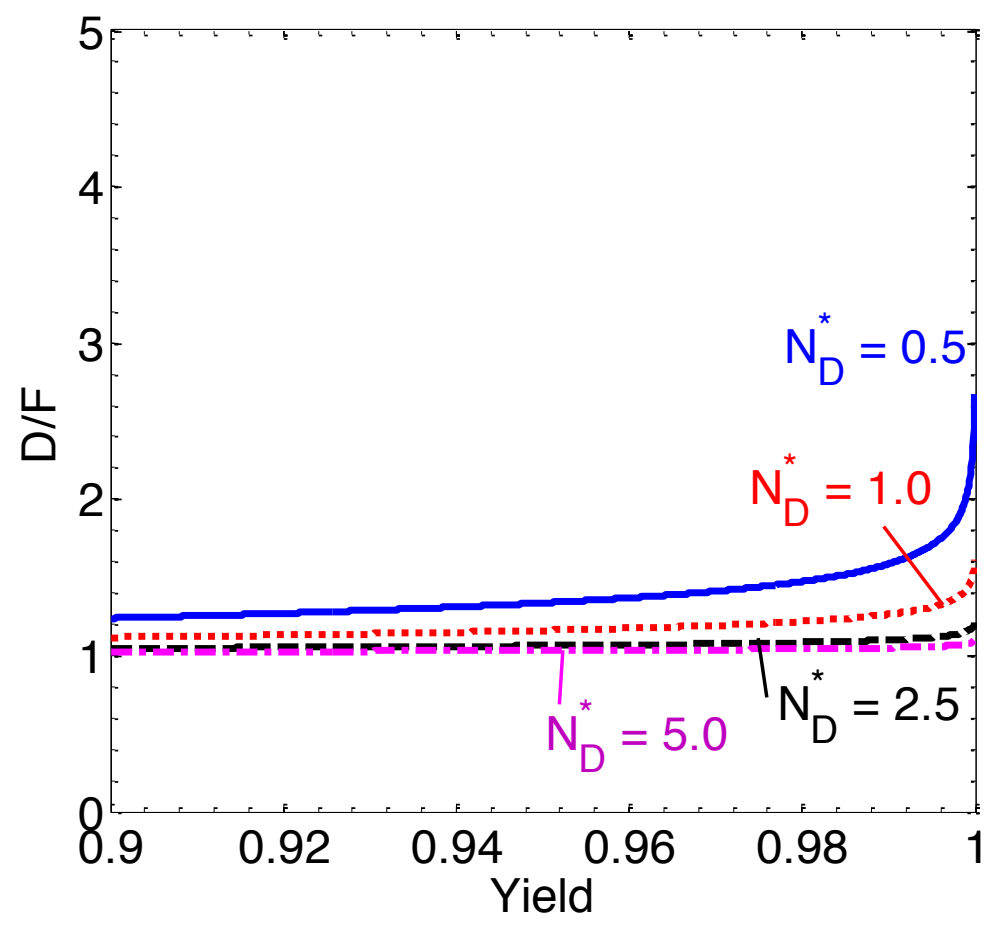

(b)

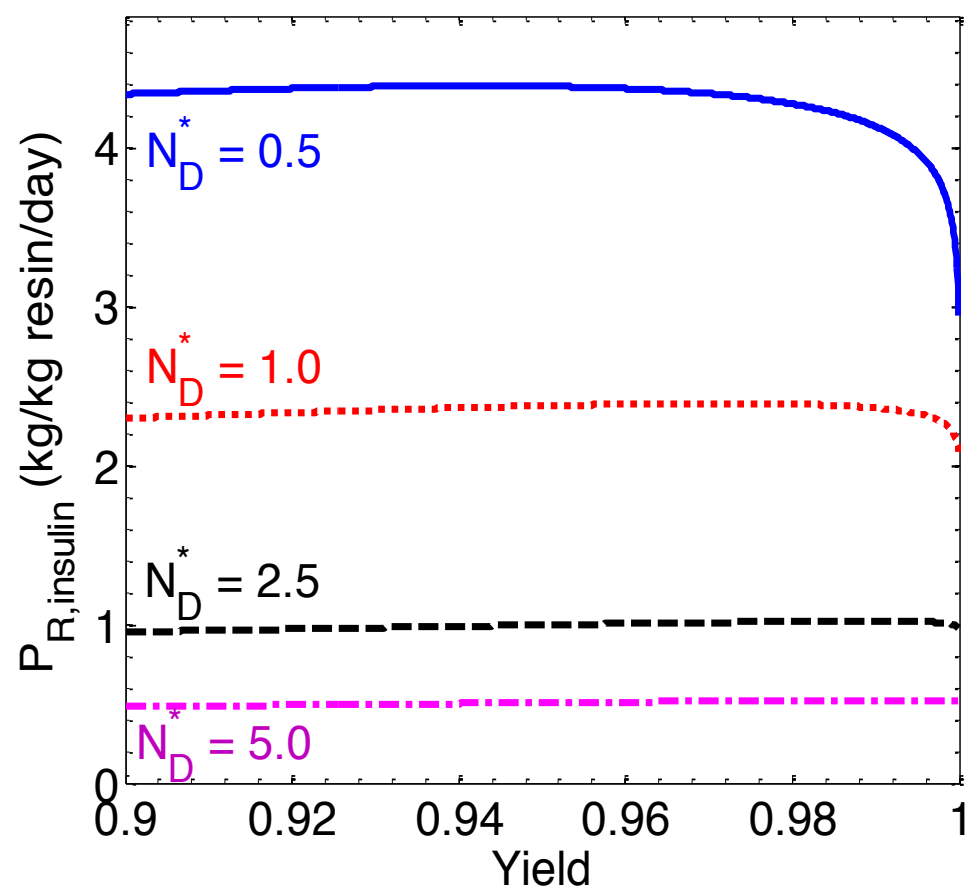


(a)

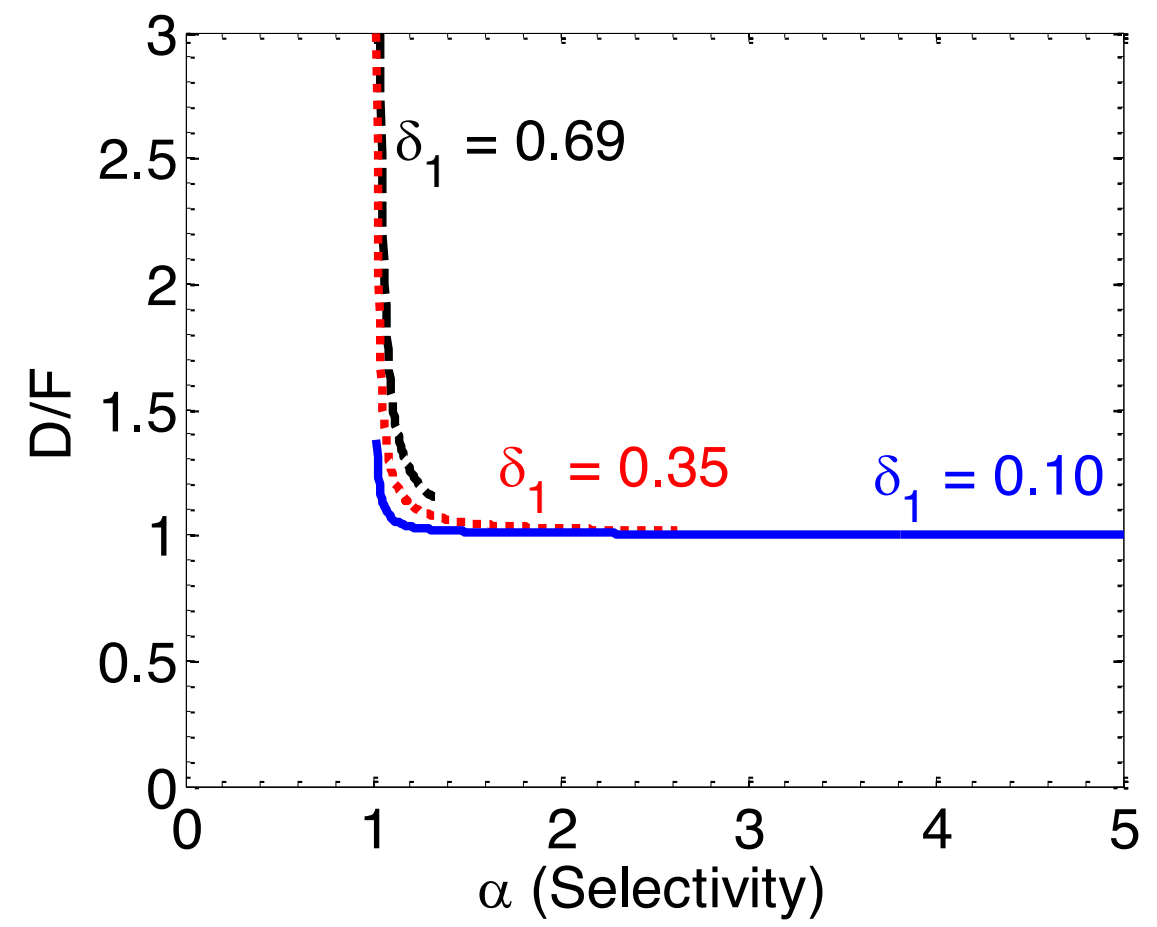

(b)

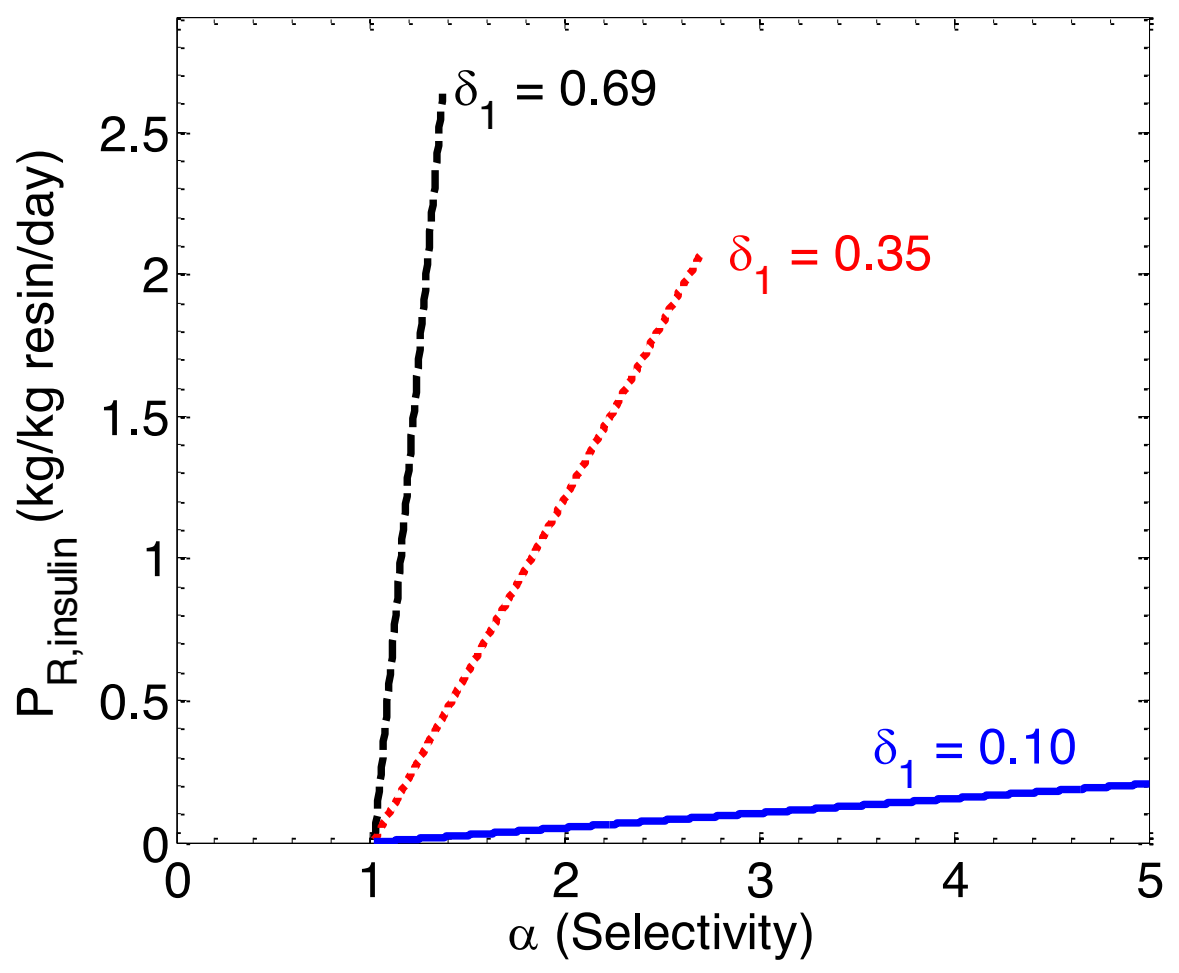


(a)

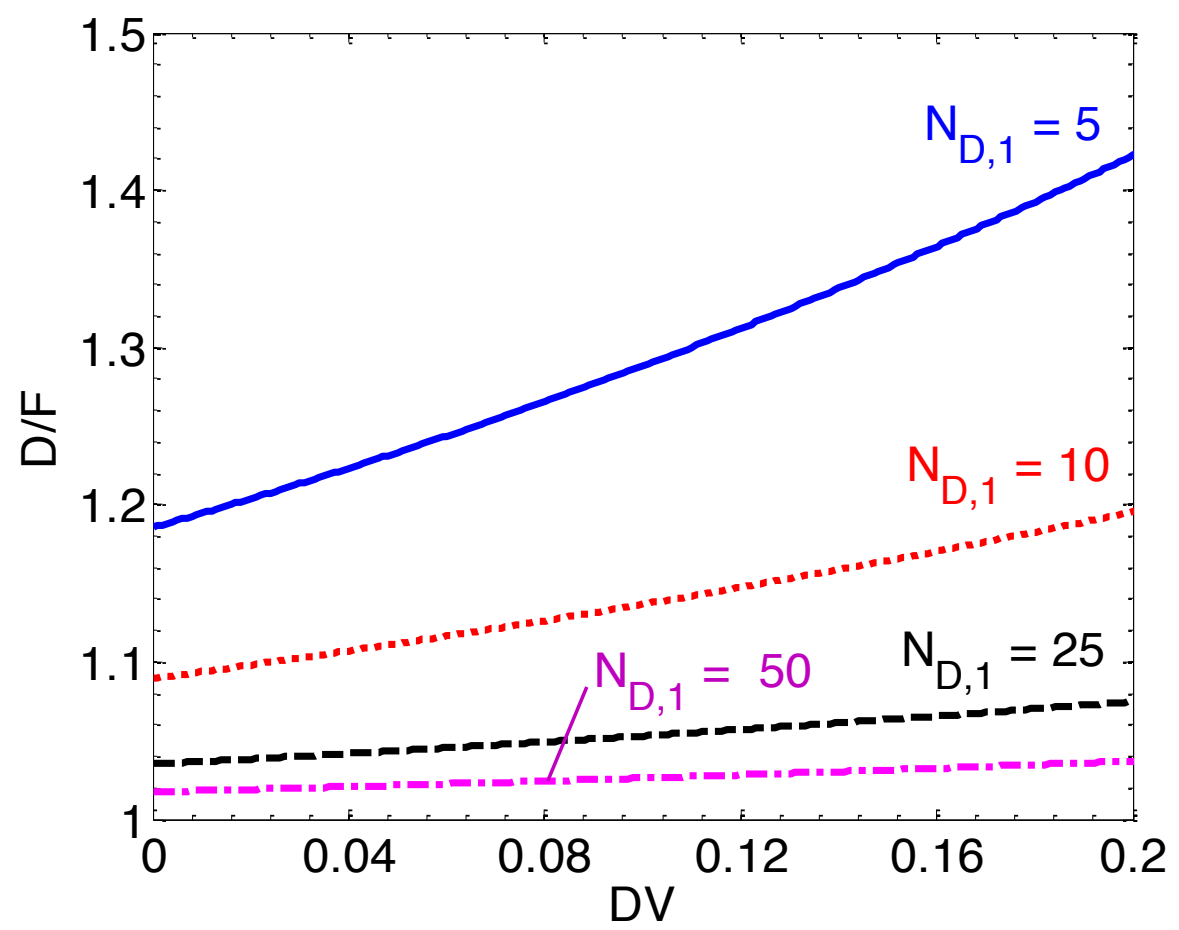

(b)

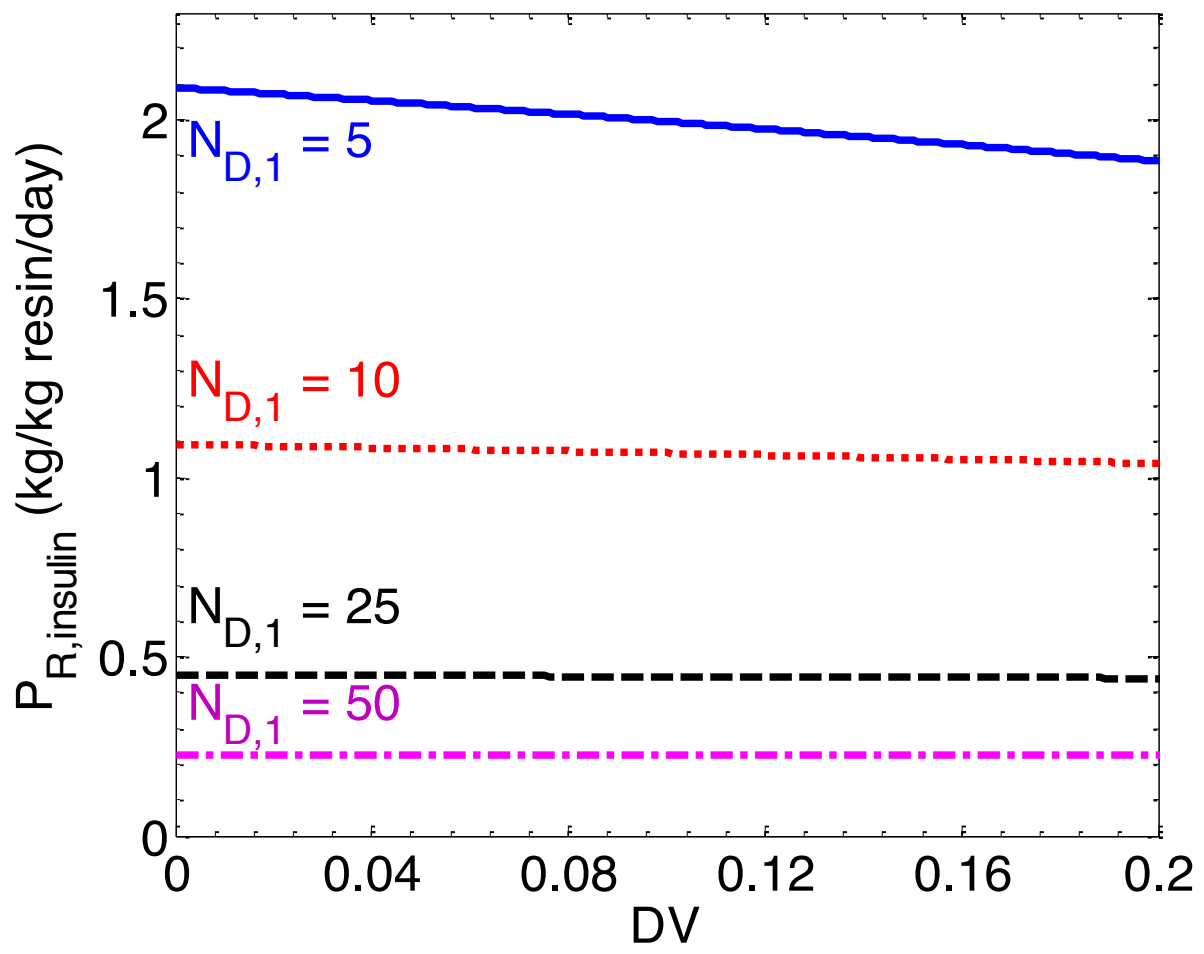


(a)

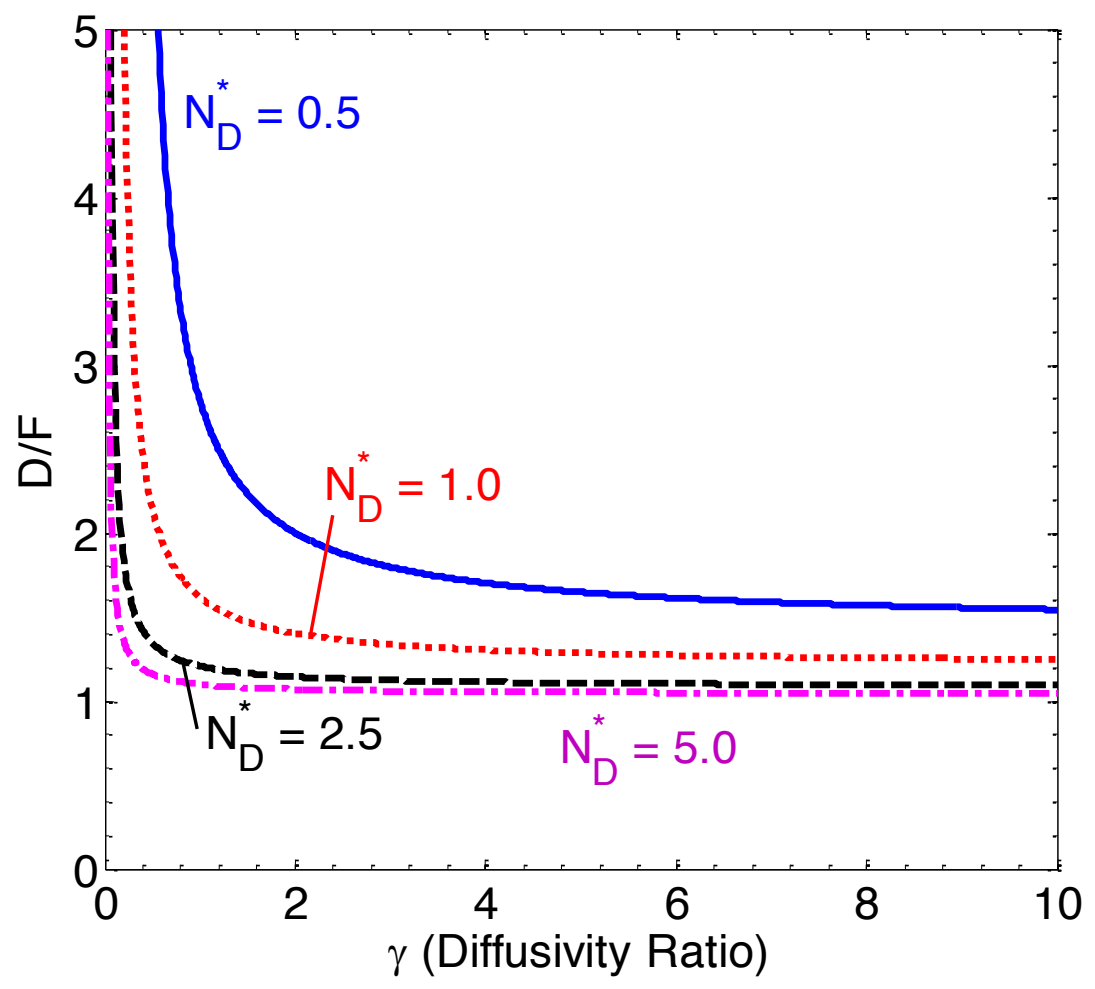

(b)

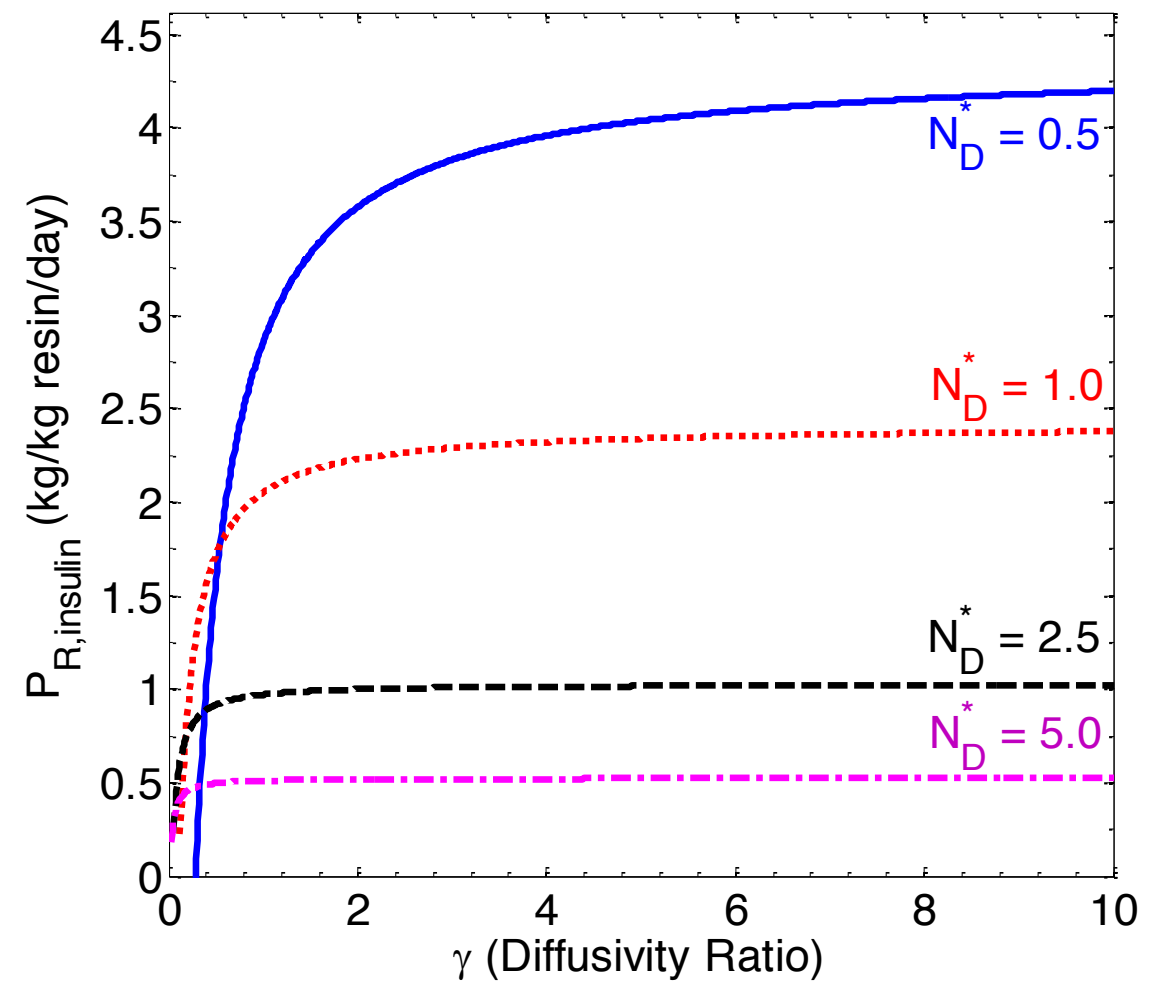


(a)

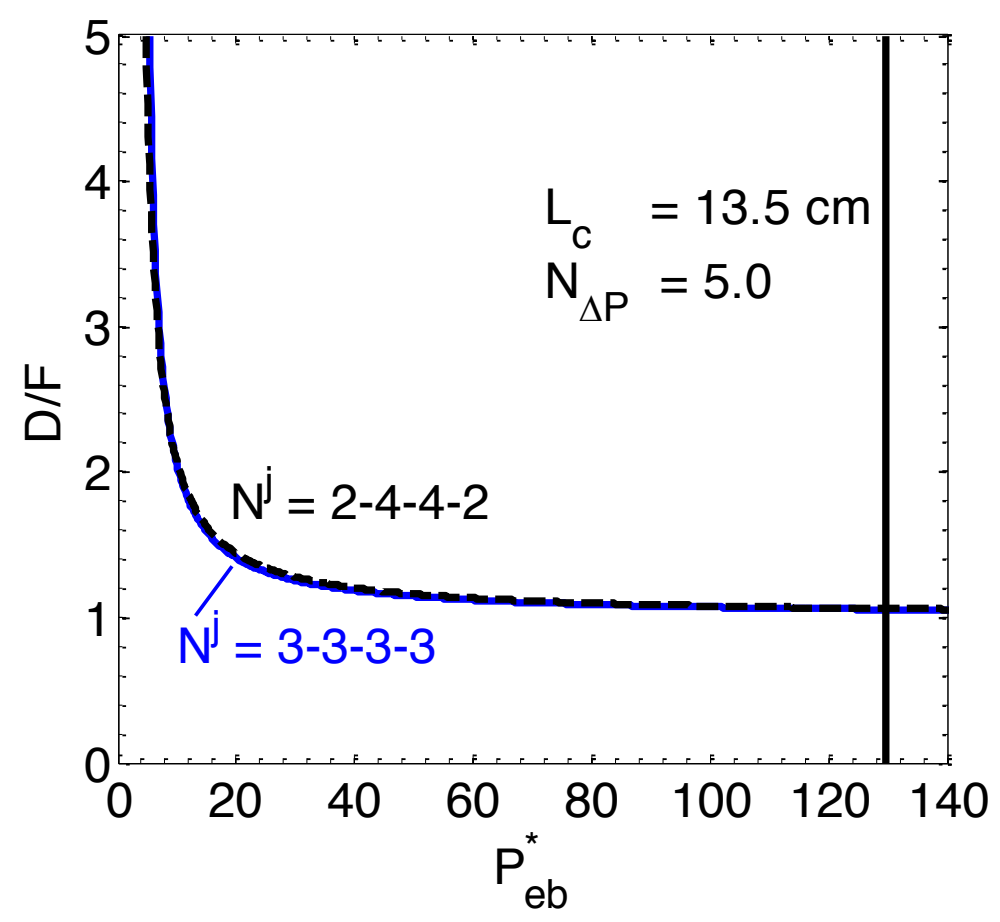

(b)

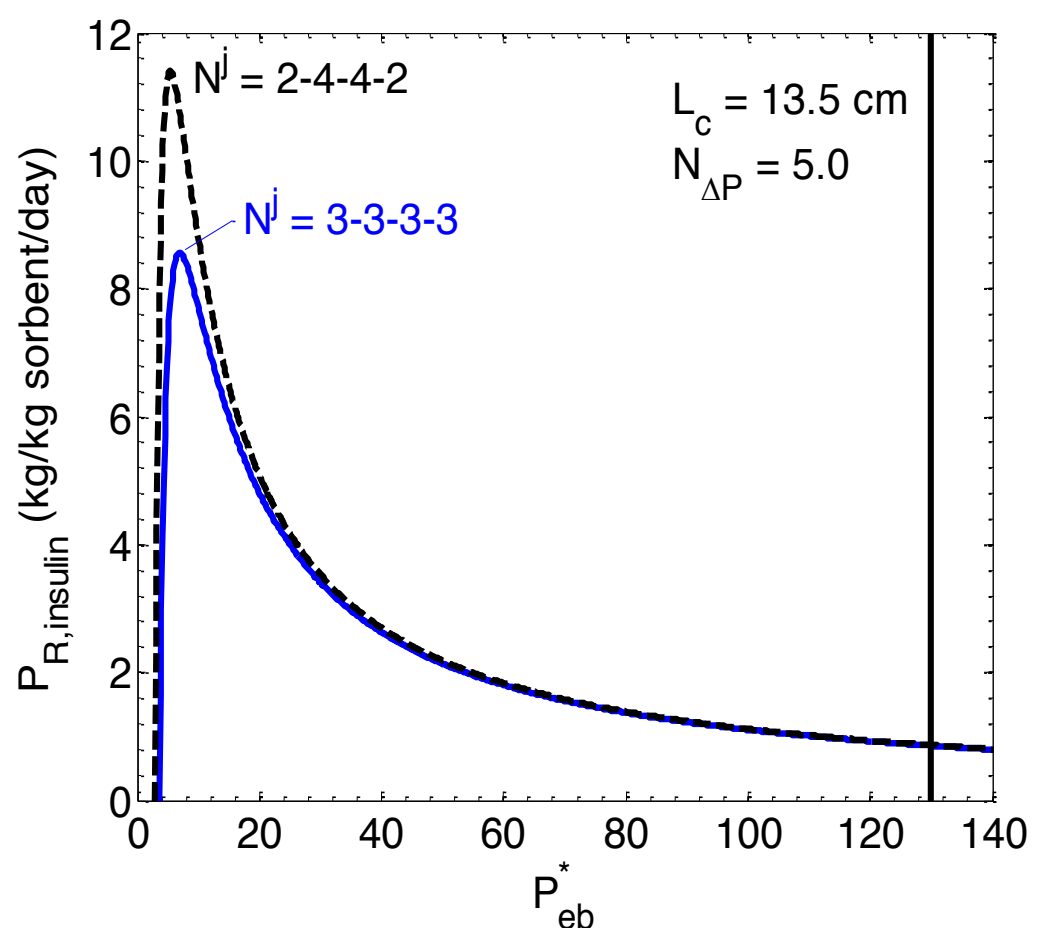


(a)

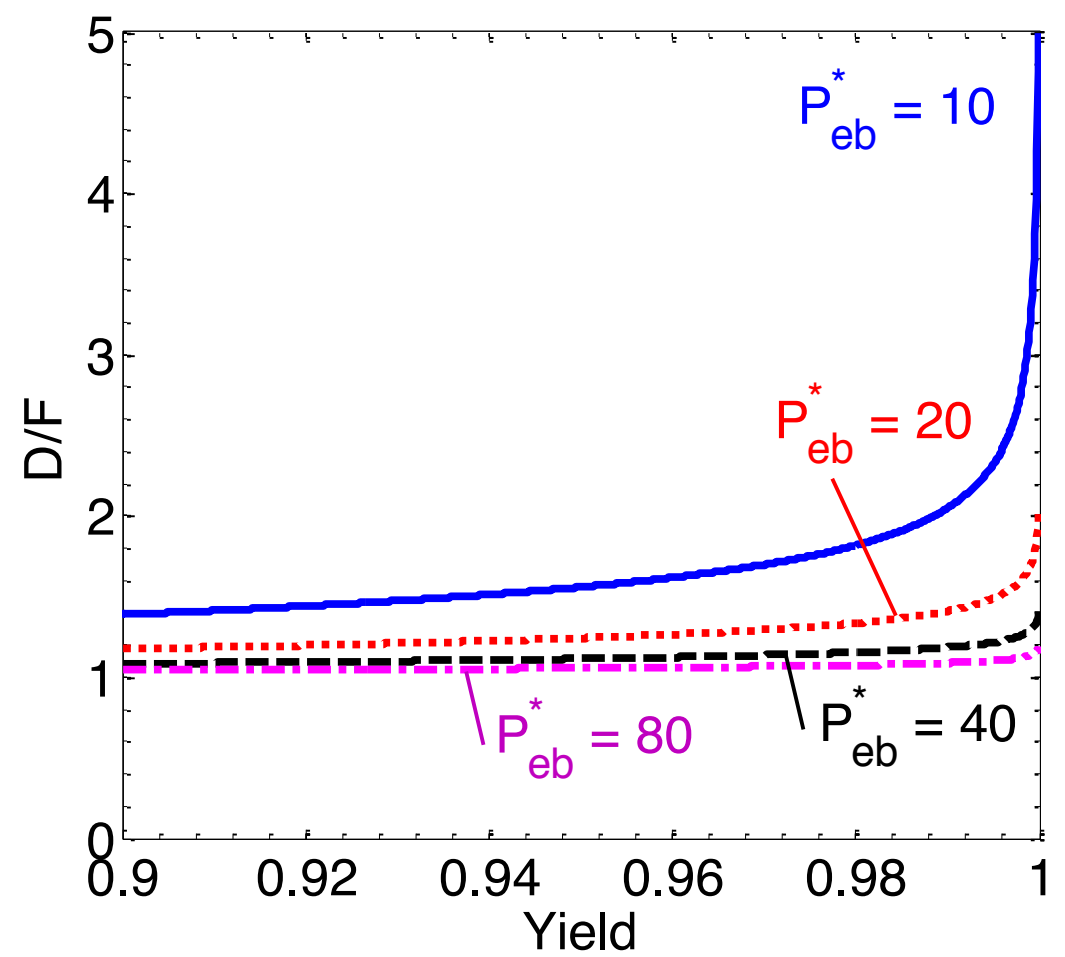

(b)

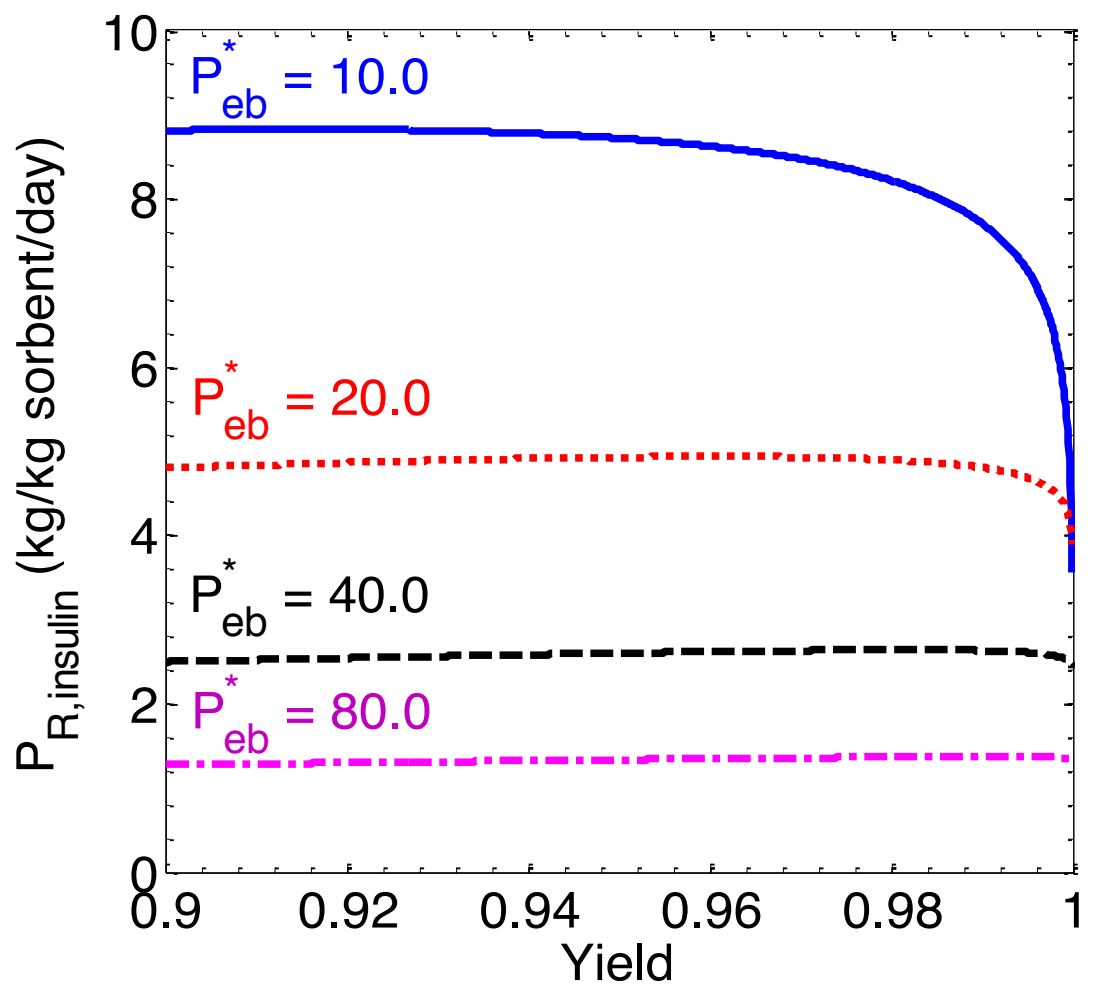


(a)

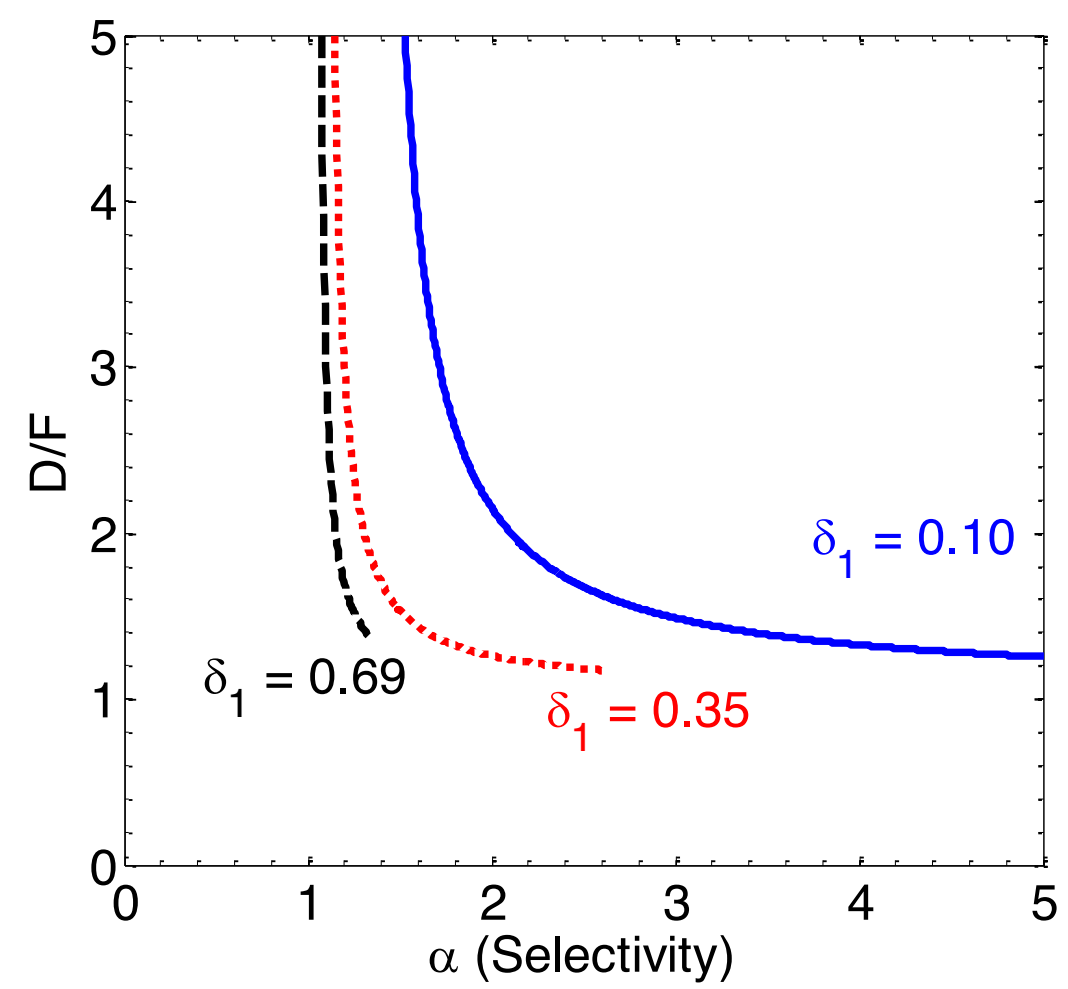

(b)

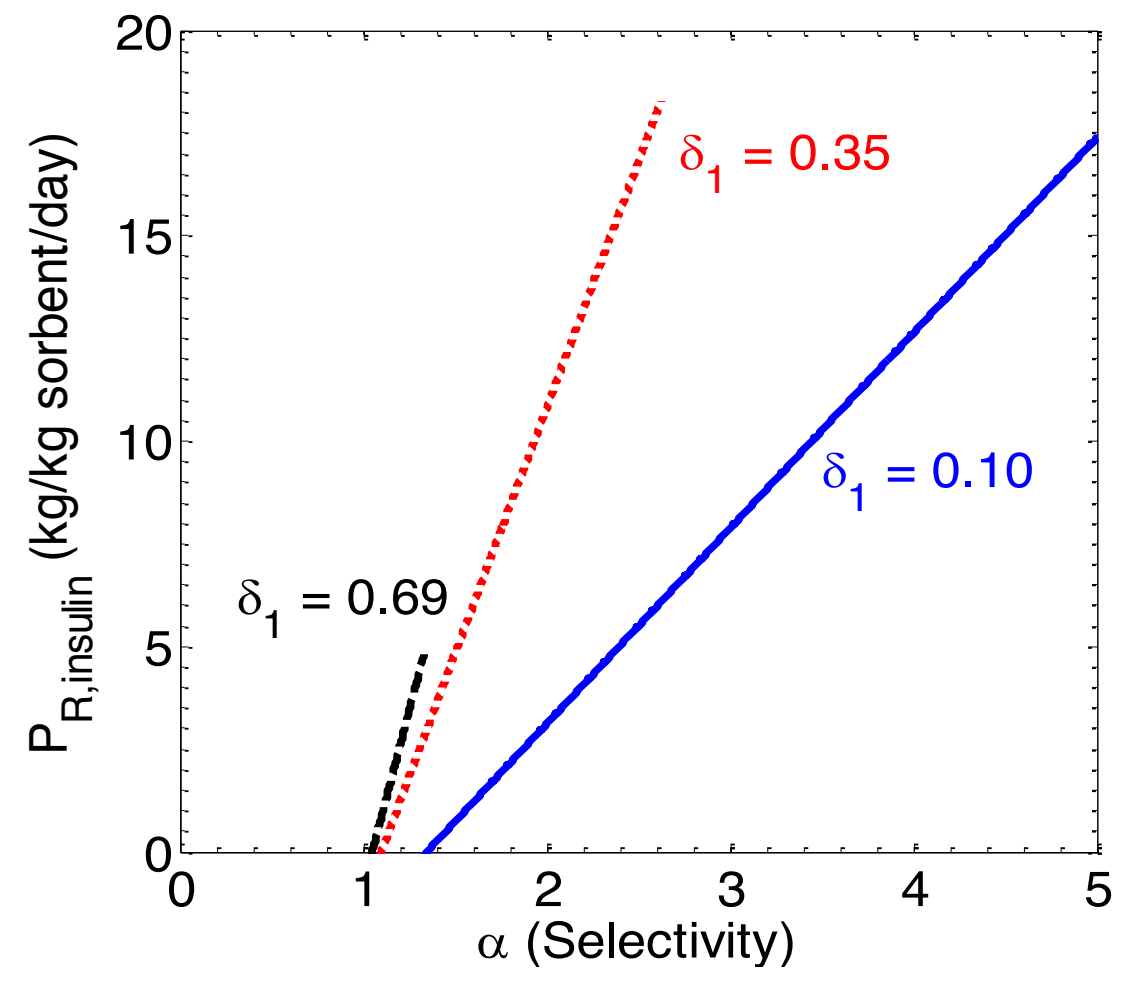




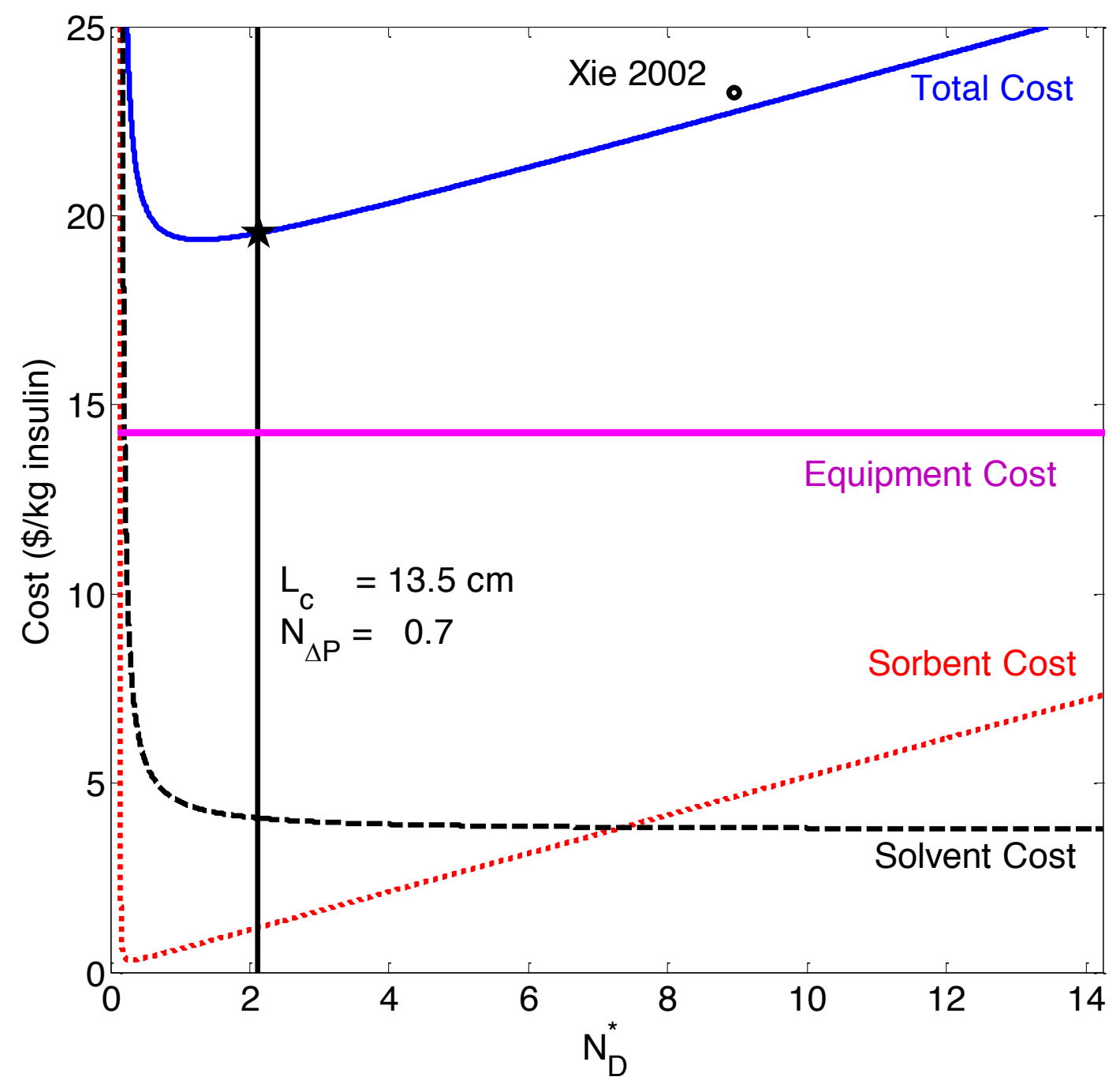


(a)

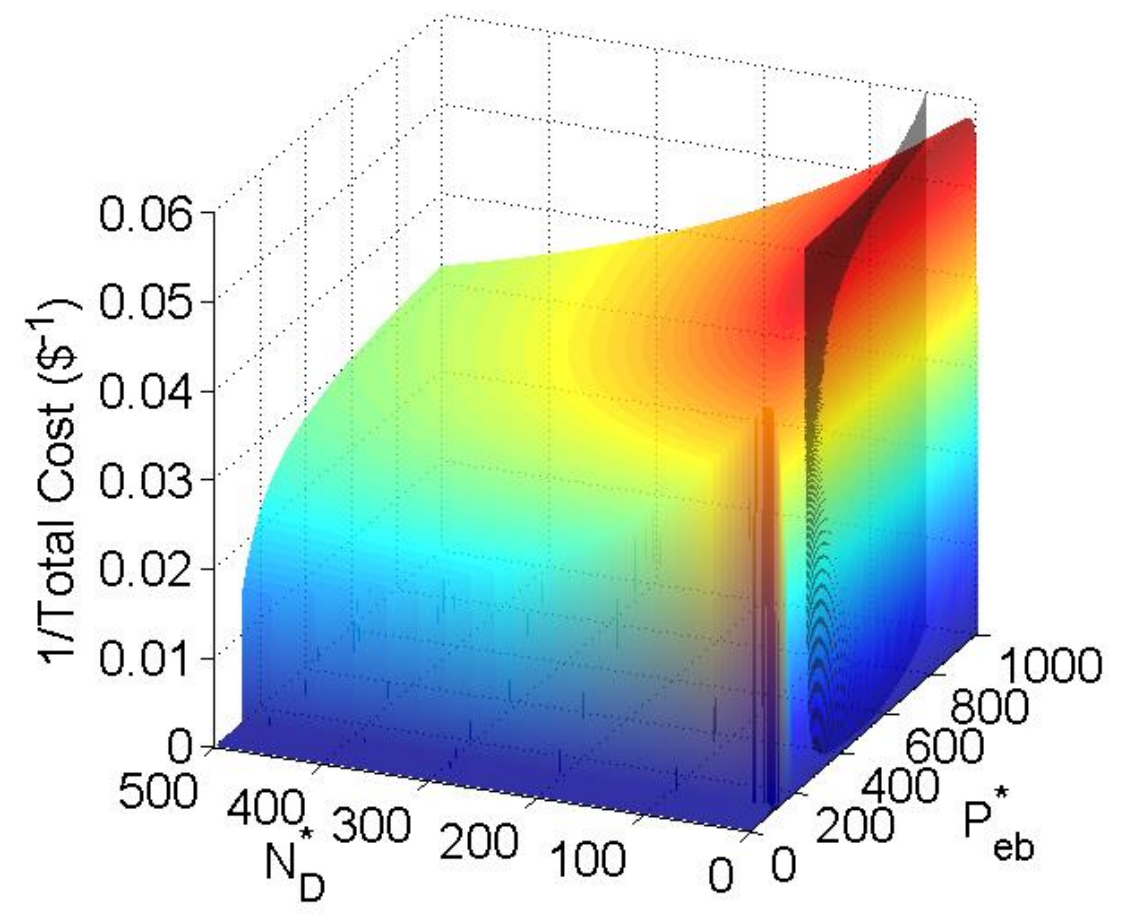

(b)

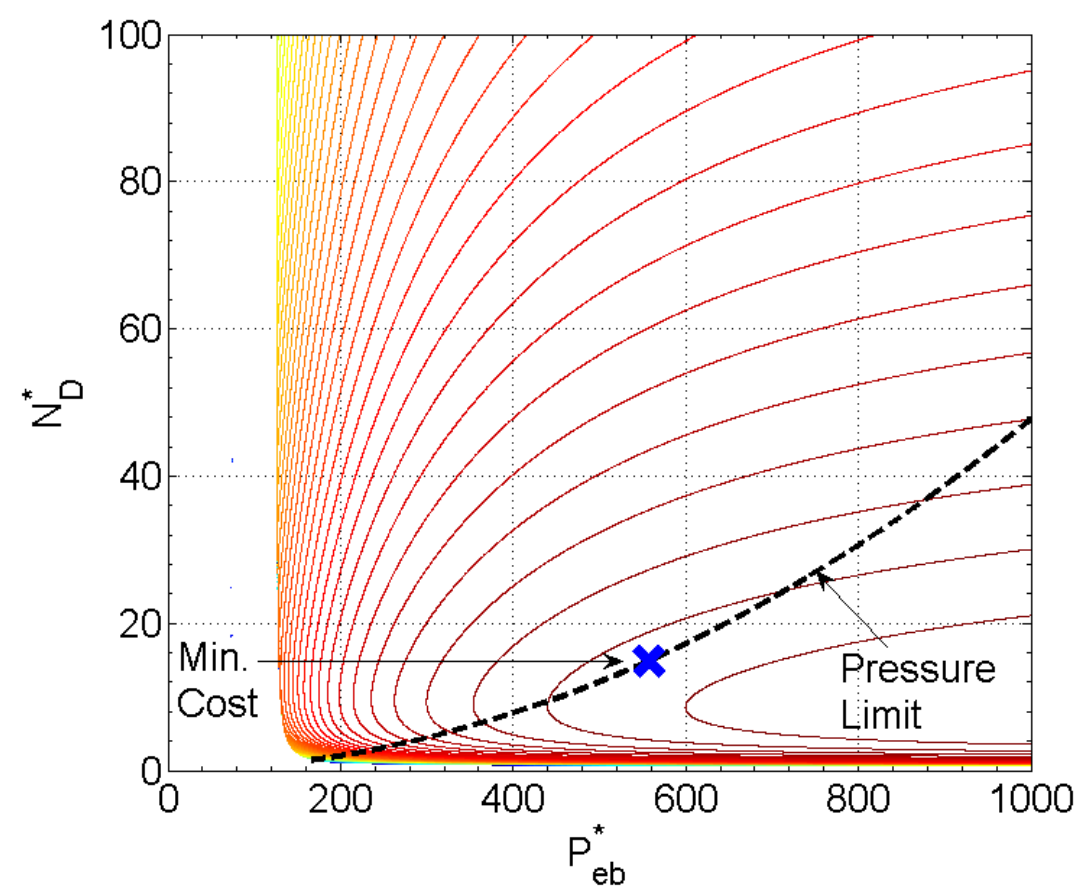


(a)

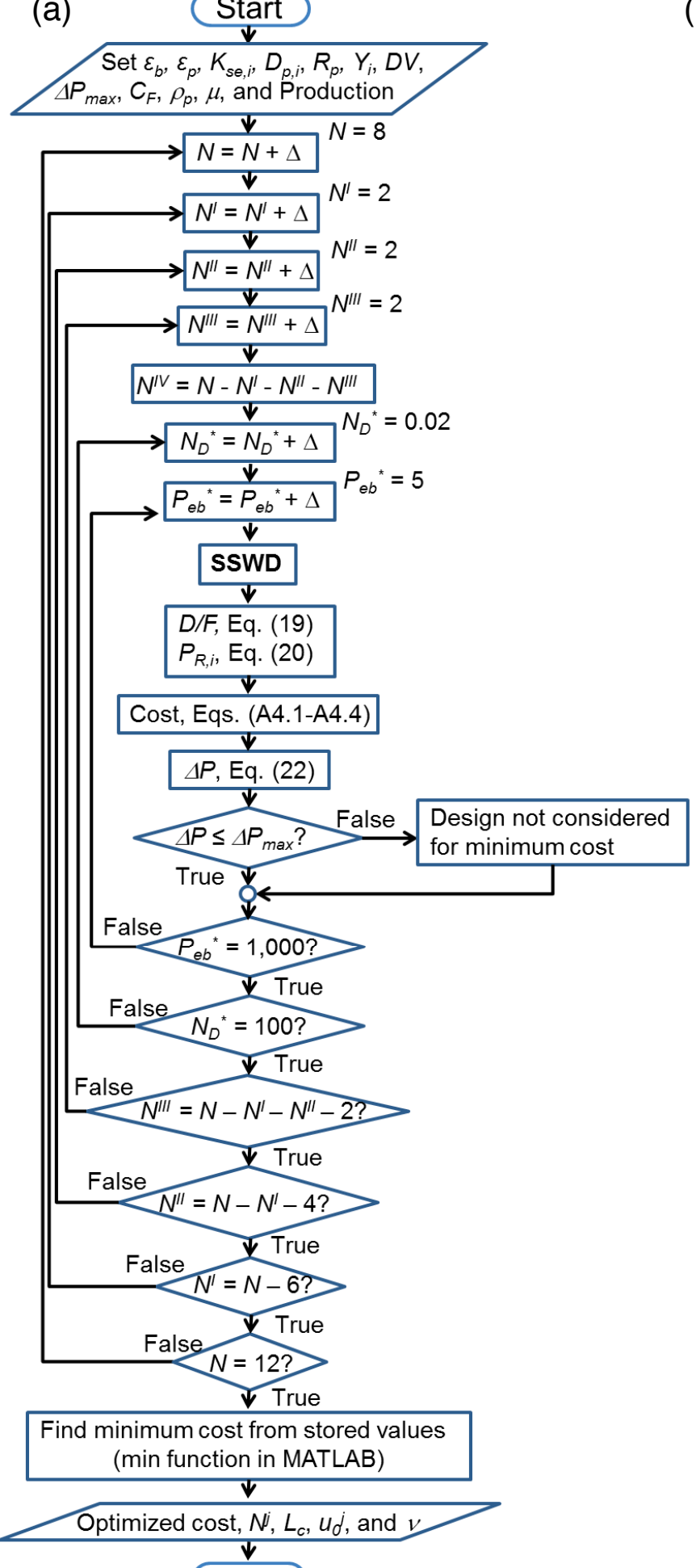

(b)

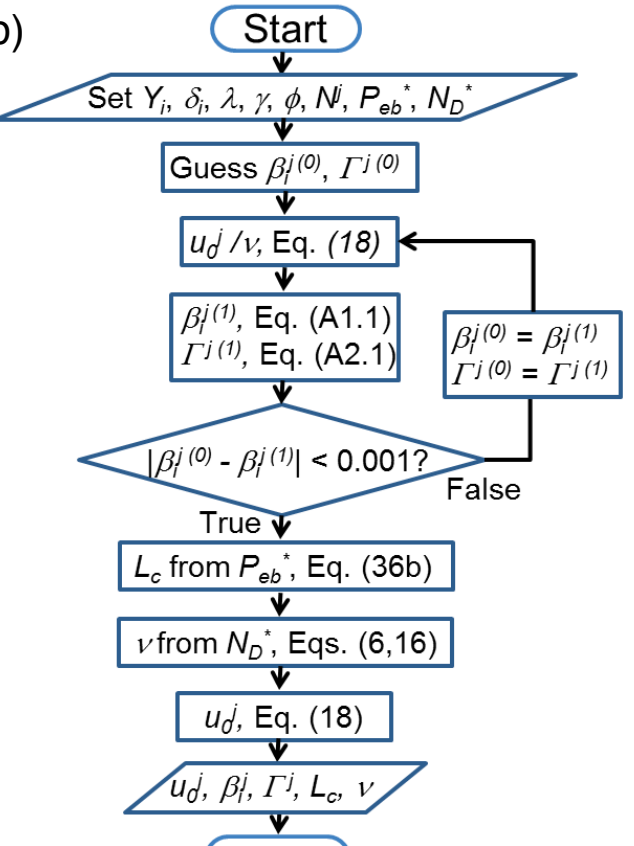

Finish

Finish 
Table 1. Dimensionless variables and groups for binary, SEC-SMB

\begin{tabular}{|c|c|c|c|}
\hline Symbol & Name & Definition & Description \\
\hline$\delta_{i}$ & $\begin{array}{l}\text { Retention } \\
\text { factor }\end{array}$ & $\delta_{i}=\varepsilon_{p} K_{s e, i}+\frac{D V}{\left(1-\varepsilon_{b}\right)}$ & $\begin{array}{l}\text { Measure of how much } \\
\text { each component is } \\
\text { retained by the sorbent }\end{array}$ \\
\hline$\alpha$ & Selectivity & $\alpha=\frac{\delta_{2}}{\delta_{1}}$ & $\begin{array}{l}\text { Ratio of retention factors: } \\
\text { greater than one for } \\
\text { separation }\end{array}$ \\
\hline$\beta_{i}^{j}$ & - & $\beta_{i}^{j}=\ln \left(\frac{C_{\max , i}^{j}}{C_{\min , i}^{j}}\right)$ & $\begin{array}{l}\text { Natural log of ratio of } \\
\text { max. conc. to min. conc. } \\
\text { of standing component } \mathrm{i} \\
\text { in zone } \mathrm{j}\end{array}$ \\
\hline$\gamma$ & $\begin{array}{l}\text { Diffusivity } \\
\text { ratio }\end{array}$ & $\gamma=\frac{D_{p, 2}}{D_{p, 1}}$ & $\begin{array}{l}\text { Ratio of intraparticle } \\
\text { diffusivities }\end{array}$ \\
\hline$\lambda$ & $\begin{array}{l}\text { Size- } \\
\text { exclusion } \\
\text { ratio }\end{array}$ & $\lambda=\frac{K_{s e, 2}}{K_{s e, 1}}$ & $\begin{array}{l}\text { Ratio of size-exclusion } \\
\text { factors; equal to } \alpha \text { if } \\
\text { there is no dead volume }\end{array}$ \\
\hline$\phi$ & Phase ratio & $\phi=\frac{1-\varepsilon_{b}}{\varepsilon_{b}}$ & $\begin{array}{l}\text { Particle phase relative to } \\
\text { bed void }\end{array}$ \\
\hline$N_{D, i}$ & - & $N_{D, i}=\frac{\phi \varepsilon_{p} K_{s e, i} D_{p, i}}{R_{p}^{2}} \frac{L_{c}}{v}=\frac{t_{s}}{t_{D, i}}$ & $\begin{array}{l}\text { Step time relative to } \\
\text { diffusion time }\end{array}$ \\
\hline$N_{D}^{*}$ & - & $N_{D}^{*}=\frac{N_{D, 1}(\alpha-1)}{\phi \delta_{1}}=\frac{N_{D, 2}(\alpha-1)}{\lambda \gamma \phi \delta_{1}}$ & $\begin{array}{l}\text { Component-independent } \\
N_{D} ; N_{D, 1} \text { as base }\end{array}$ \\
\hline$N_{\Delta P}$ & - & $N_{\Delta P}=\frac{\Delta P_{\max } R_{p}^{2}}{37.5 \phi \mu L_{c} v}=\frac{t_{s}}{t_{c}^{I}}$ & $\begin{array}{l}\text { Step time relative to } \\
\text { pressure-limited } \\
\text { convection time }\end{array}$ \\
\hline$N_{\Delta P, \text { diff }}$ & - & $N_{\Delta P, \text { diff }}=\frac{\Delta P_{\max } R_{p}{ }^{4}}{37.5 \phi \mu D_{p, 1} L_{c}^{2}}=\frac{t_{D, 1}}{t_{c}^{I}}$ & $\begin{array}{l}\text { Diffusion time relative to } \\
\text { pressure-limited } \\
\text { convection time }\end{array}$ \\
\hline$P_{e b}^{j}$ & Peclet number & $P_{e b}^{j}=\frac{v L_{c}}{E_{b}^{j}}=\frac{t_{D a x}}{t_{s}}$ & $\begin{array}{l}\text { Axial dispersion time } \\
\text { relative to step time }\end{array}$ \\
\hline$\Gamma^{j}$ & $\begin{array}{l}\text { Axial } \\
\text { dispersion } \\
\text { ratio }\end{array}$ & $\Gamma^{j}=\frac{E_{b}^{j}}{E_{b}^{I V}}$ & $\begin{array}{l}\text { Ratio of axial dispersion } \\
\text { coefficients of zone } \mathrm{j} \text { and } \\
\text { zone IV }\end{array}$ \\
\hline$P_{e b}^{*}$ & - & $P_{e b}^{*}=\phi\left(\delta_{2}-\delta_{1}\right) P_{e b}^{j} \Gamma^{j}$ & $\begin{array}{l}\text { Zone-independent Peclet } \\
\text { number, Zone IV as base }\end{array}$ \\
\hline
\end{tabular}


Table 2. SSWD equations for binary SEC-SMB.

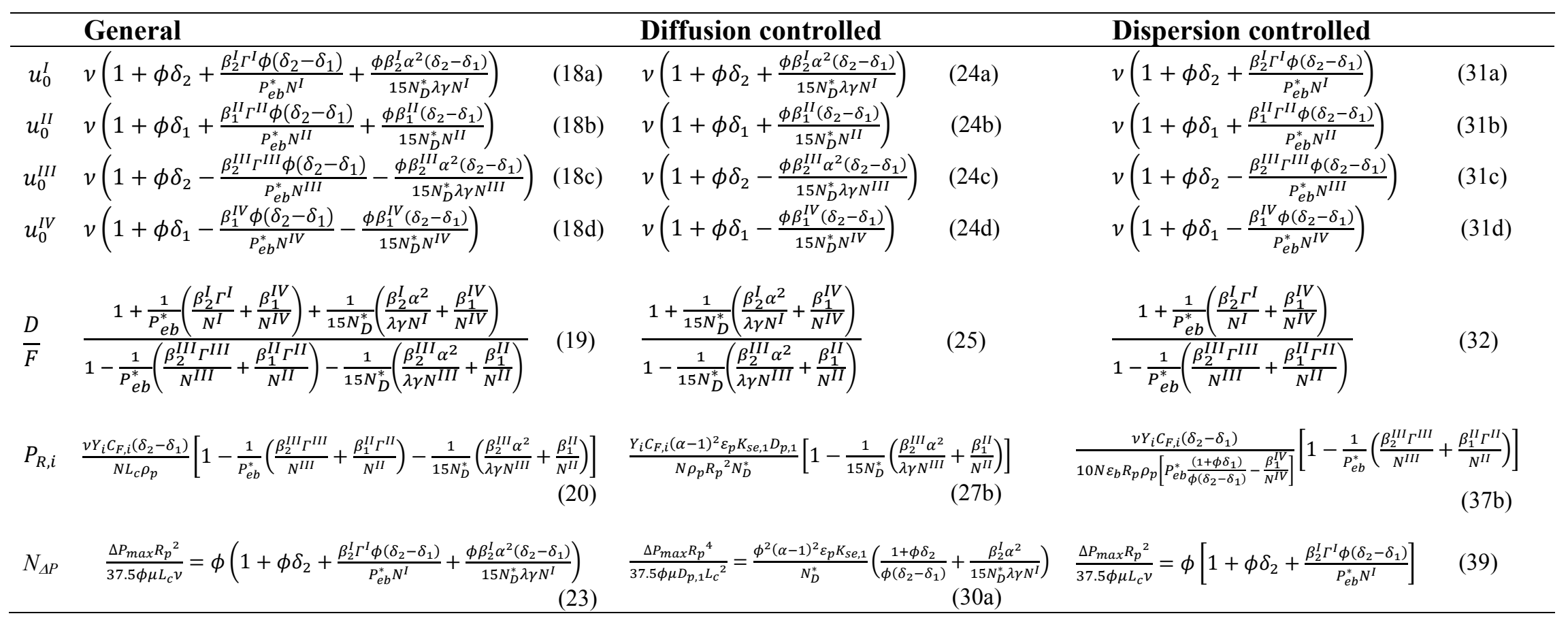


Table 3. Table of parameters for three literature cases. Complete lists of parameters in Tables B.1-B.3.

\begin{tabular}{|c|c|c|c|}
\hline & Case 1 & Case 2 & Case $3^{*}$ \\
\hline Authors & Liang and Liang & Houwing et al. & Xie et al. \\
\hline Year & 2012 & 2003 & 2002 \\
\hline Component 1 & 20,000 MW PEG & BSA & Insulin \\
\hline Component 2 & 1,500 MW PEG & Myoglobin & $\mathrm{ZnCl}_{2}$ \\
\hline Design & Triangle & Triangle & SWD \\
\hline $\begin{array}{l}\text { Yield (\%) } \\
\text { (Comp. 1, Comp. 2) }\end{array}$ & $\begin{array}{l}\text { 1A: } 65.5,99.9 \\
\text { 1B: } 99.9,99.9 \\
\text { 1C: } 99.9,70.7\end{array}$ & $\begin{array}{l}\text { 2A: } 46.2,90.0 \\
\text { 2B: } 60.5,90.0 \\
\text { 2C: } 71.3,82.5 \\
\text { 2D: } 86.7,60.0 \\
\text { 2E: } 99.8,40.5\end{array}$ & $99.0,99.0$ \\
\hline Configuration & $2-2-2$ & $2-2-2-2$ & $2-3-3-2$ \\
\hline$L_{c}(\mathrm{~cm})$ & 30 & 8.9 & 13.7 \\
\hline$I D(\mathrm{~cm})$ & 0.75 & 1.0 & 5.1 \\
\hline$R_{p}(\mu \mathrm{m})$ & 8.5 & 100 & 54 \\
\hline$N_{D}^{*}$ & $\sim 4,600$ & 0.36 & 8.9 \\
\hline$P_{e b}{ }^{*}\left(L_{c} / 10 \varepsilon_{b} R_{p}\right)$ & $1,751(9,688)$ & $35.8(228)$ & $145(725)$ \\
\hline
\end{tabular}

"Ring B of two ring tandem SMB 
Table 4. Fixed parameters for insulin parametric study unless otherwise specified.

\begin{tabular}{|c|c|c|c|c|c|c|c|}
\hline $\boldsymbol{R}_{p}^{*}(\mu \mathrm{m})$ & $\varepsilon_{b}$ & $\varepsilon_{p}$ & $\phi$ & $D V(\%)$ & $Y(\%)$ & \multirow{2}{*}{\multicolumn{2}{|c|}{$\begin{array}{c}\boldsymbol{E}_{\boldsymbol{b}, i}^{j} \\
\text { Chung and Wen } \\
\text { Correlation }\end{array}$}} \\
\hline 54 & 0.35 & 0.89 & 1.86 & 1.9 & 99 & & \\
\hline \multicolumn{4}{|l|}{ Component } & \multicolumn{2}{|c|}{$\begin{array}{c}D_{p} \\
\left(\mathrm{~cm}^{2} / \min \right)\end{array}$} & $\boldsymbol{K}_{s e}$ & $\delta$ \\
\hline \multirow{3}{*}{\multicolumn{4}{|c|}{$\begin{array}{l}\text { High Molecular Weight Proteins (HMWP) } \\
\text { Insulin } \\
\text { Zinc Chloride }\end{array}$}} & \multicolumn{2}{|c|}{$2.00 \times 10^{-5}$} & 0.19 & 0.198 \\
\hline & & & & \multirow{2}{*}{\multicolumn{2}{|c|}{$\begin{array}{l}2.29 \times 10^{-5} \\
1.65 \times 10^{-4}\end{array}$}} & 0.74 & 0.688 \\
\hline & & & & & & 0.99 & 0.910 \\
\hline \multirow{2}{*}{\multicolumn{3}{|c|}{ A (Insulin / HMWP) }} & $\alpha$ & \multirow{2}{*}{\multicolumn{2}{|c|}{$\gamma$}} & & $\gamma / a$ \\
\hline & & & 3.47 & 1.15 & & & 0.33 \\
\hline \multicolumn{3}{|c|}{$\mathrm{B}\left(\mathrm{ZnCl}_{2} /\right.$ Insulin $)$} & 1.32 & \multicolumn{2}{|c|}{7.21} & & 5.46 \\
\hline $\begin{array}{c}C_{F, i}(\mathrm{~g} / \mathrm{L}) \\
54\end{array}$ & \multicolumn{4}{|c|}{$\begin{array}{c}\rho_{p}(\mathrm{~kg} \text { sorbent } / \mathrm{L} \text { particle volume }) \\
0.12\end{array}$} & $\begin{aligned} & \mu(c \\
& 2 .\end{aligned}$ & & $\begin{array}{l}\Delta P_{\max }^{*}(\mathrm{psi}) \\
1.5\end{array}$ \\
\hline
\end{tabular}

"Sephadex G50 
Table 5. Comparison of batch, SMB, and cost optimized SMB

\begin{tabular}{|c|c|c|c|c|c|}
\hline \multirow{2}{*}{$\begin{array}{l}\text { Parameter } \\
(5,000 \mathrm{~kg} \text { insulin / year })\end{array}$} & \multirow{2}{*}{ Batch $^{\text {b }}$} & \multirow{2}{*}{ Xie (2002) } & \multicolumn{3}{|c|}{ Optimized SMB ${ }^{\mathrm{a}}$} \\
\hline & & & $\operatorname{Ring} A^{b}$ & Ring B & Overall $^{b}$ \\
\hline Overall Yield (\%) & 89 & 99 & 99 & 99 & 98 \\
\hline Product Concentration $(\mathrm{g} / \mathrm{L})$ & 45.0 & 59.0 & 61.9 & 47.8 & 47.8 \\
\hline Column Configuration & 12 in series & $\begin{array}{l}\text { A: } 2-2-4-2 \\
\text { B: } 2-3-3-2\end{array}$ & $2-2-2-2$ & $2-2-2-2$ & $\begin{array}{l}\text { A: } 2-2-2-2 \\
\text { B: } 2-2-2-2\end{array}$ \\
\hline Feed Flowrate $(\mathrm{mL} / \mathrm{min})$ & 119 (each) & 109 & 109 & - & 109 \\
\hline Column Length (cm) & 15 & $\begin{array}{l}\text { A: } 13.7 \\
\text { B: } 13.7\end{array}$ & 15.9 & 11.2 & $\begin{array}{l}\text { A: } 15.9 \\
\text { B: } 11.2\end{array}$ \\
\hline Diameter $(\mathrm{cm})$ & $\begin{array}{c}45 \\
\text { (12 units) }\end{array}$ & $\begin{array}{l}\text { A: } 47.9 \\
\text { B: } 58.6\end{array}$ & 20.4 & 29.8 & $\begin{array}{l}\text { A: } 20.4 \\
\text { B: } 29.8\end{array}$ \\
\hline$N_{D}{ }^{*}$ & - & $\begin{array}{l}\text { A: } 6.0 \\
\text { B: } 8.9\end{array}$ & 14.9 & 1.48 & $\begin{array}{l}\text { A: } 14.9 \\
\text { B: } 1.48\end{array}$ \\
\hline$P_{e b}{ }^{*}$ & - & $\begin{array}{l}\text { A: } 145 \\
\text { B: } 145\end{array}$ & 560 & 108 & $\begin{array}{l}\text { A: } 560 \\
\text { B: } 108\end{array}$ \\
\hline $\begin{array}{l}\text { Solvent consumption } \\
\text { (L/kg insulin) }\end{array}$ & 150.0 & 42.0 & 28.0 & 22.8 & 50.8 \\
\hline $\begin{array}{l}\text { Productivity } \\
\text { (kg/kg sorbent/day) }\end{array}$ & 0.05 & 0.14 & 4.47 & 2.80 & 1.69 \\
\hline Equip. Cost ( $\$ / \mathrm{kg}$ insulin) & $35.71(39 \%)$ & $29.76(72 \%)$ & 14.29 & 14.29 & $28.58(81 \%)$ \\
\hline Solv. Cost (\$/kg insulin) & $15.00(17 \%)$ & $3.96(10 \%)$ & 2.80 & $2.28^{\mathrm{d}}$ & $5.08(15 \%)$ \\
\hline Resin Cost (\$/kg insulin) & $40.07(44 \%)$ & $7.41(18 \%)$ & 0.43 & 0.79 & $1.22(4 \%)$ \\
\hline Total Cost (\$/kg insulin) & 90.78 & 41.43 & 17.58 & 17.36 & 34.94 \\
\hline $\begin{array}{l}{ }^{\mathrm{a}} \text { Under constraint that there } \\
{ }^{b} \text { Feed concentration of insul } \\
\text { cFeed concentration of insul } \\
{ }^{\mathrm{d}} \text { Ring B solvent cost only in }\end{array}$ & $88.5 \mathrm{~g} / \mathrm{L}$. & 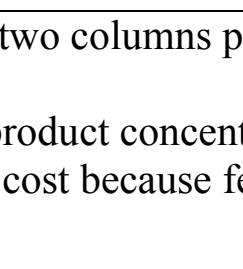 & r zone & Ring A & 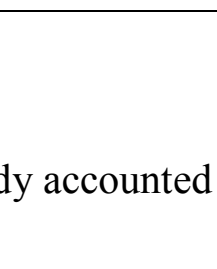 \\
\hline
\end{tabular}


Table 6. Effects of material properties, pressure limit, and dead volume on Ring B optimization.

\begin{tabular}{|c|c|c|c|c|c|c|c|c|}
\hline $\begin{array}{c}\text { Parameter } \\
\text { Changed }\end{array}$ & $\begin{array}{c}\text { New } \\
\text { Parameter } \\
\text { Values }\end{array}$ & $D / F$ & $\begin{array}{c}\text { Solvent } \\
\text { Cost } \\
(\$ / k g \\
\text { insulin) }\end{array}$ & $\begin{array}{c}L_{c} \\
(\mathbf{c m})\end{array}$ & $\begin{array}{c}I D \\
(\mathrm{~cm})\end{array}$ & $\begin{array}{c}P_{R} \\
\text { (kg insulin/ } \\
\begin{array}{c}\text { kg resin/ } \\
\text { day) }\end{array}\end{array}$ & $\begin{array}{c}\text { Sorbent } \\
\text { Cost } \\
(\$ / k g \\
\text { insulin) }\end{array}$ & $\begin{array}{c}\text { Total } \\
\text { Cost }^{\mathrm{e}} \\
(\$ / \mathrm{kg} \\
\text { insulin) }\end{array}$ \\
\hline \multicolumn{2}{|c|}{ Base Case (Table 5) } & 1.39 & 2.27 & 11.4 & 29.6 & 2.79 & 0.79 & 17.35 \\
\hline \multirow{2}{*}{$\Delta P_{\max }(\mathrm{psi})$} & 50 & 1.32 & 2.16 & 61.5 & 11.9 & 3.23 & 0.68 & 17.13 \\
\hline & 75 & 1.32 & 2.15 & 75.3 & 10.7 & 3.23 & 0.68 & 17.12 \\
\hline$D V$ & 0 & 1.38 & 2.25 & 11.2 & 29.0 & 2.96 & 0.74 & 17.28 \\
\hline$(\% \mathrm{CV})$ & 10 & 1.46 & 2.38 & 11.9 & 31.9 & 2.33 & 0.95 & 17.62 \\
\hline \multirow[b]{2}{*}{$\varepsilon_{b}$} & 0.3 & 1.40 & 2.29 & 8.4 & 33.3 & 2.79 & 0.79 & 17.37 \\
\hline & 0.4 & 1.40 & 2.28 & 14.8 & 26.8 & 2.85 & 0.77 & 17.34 \\
\hline \multirow{2}{*}{$\varepsilon_{p}$} & 0.50 & 1.53 & 2.49 & 12.2 & 35.7 & 1.80 & 1.22 & 18.00 \\
\hline & 0.95 & 1.38 & 2.25 & 11.4 & 29.1 & 2.91 & 0.76 & 17.30 \\
\hline \multirow[b]{2}{*}{$\gamma$} & 1 & 1.50 & 2.46 & 13.7 & 33.5 & 1.83 & 1.21 & 17.96 \\
\hline & 100 & 1.37 & 2.24 & 10.9 & 28.8 & 3.11 & 0.71 & 17.24 \\
\hline \multirow{2}{*}{$K_{s e, 1}$} & 0.010 & 1.07 & 1.74 & 8.3 & 12.0 & 23.33 & 0.09 & 16.12 \\
\hline & 0.100 & 1.07 & 1.75 & 8.2 & 12.6 & 21.36 & 0.10 & 16.14 \\
\hline \multirow{2}{*}{$R_{p}(\mu \mathrm{m})$} & 25 & 1.26 & 2.06 & 3.7 & 35.9 & 5.85 & 0.38 & 16.73 \\
\hline & 100 & 1.61 & 2.63 & 29.9 & 26.8 & 1.31 & 1.68 & 18.61 \\
\hline$\Delta P_{\max }, D V$ & $150,0.0$ & & & & & & & \\
\hline $\begin{array}{l}\varepsilon_{b}, \varepsilon_{p} \\
\gamma, K_{s e, 1} \\
R_{p}\end{array}$ & $\begin{array}{c}0.3,0.95 \\
100,0.01 \\
25\end{array}$ & 1.01 & 1.65 & 10.4 & 3.7 & 187.49 & 0.01 & 15.95 \\
\hline
\end{tabular}

${ }^{\mathrm{e}}$ The equipment cost does not change between cases. 
Table B.1. Extended table of parameters for Case 1.

\begin{tabular}{|c|c|c|c|}
\hline & $\mathbf{1 A}$ & $1 B$ & $1 \mathrm{C}$ \\
\hline Authors & & \multicolumn{2}{|c|}{ Liang and Liang } \\
\hline Year & & \multicolumn{2}{|c|}{2012} \\
\hline Component 1 & & \multicolumn{2}{|c|}{ 20,000 MW PEG } \\
\hline Component 2 & & \multicolumn{2}{|c|}{ 1,500 MW PEG } \\
\hline Design & & \multicolumn{2}{|c|}{ Triangle } \\
\hline$\varepsilon_{p}$ & & \multicolumn{2}{|l|}{0.80} \\
\hline$\varepsilon_{b}$ & & \multicolumn{2}{|l|}{0.364} \\
\hline$K_{s e, 1}, K_{s e, 2}$ & & \multicolumn{2}{|l|}{$0.38,0.57$} \\
\hline$\delta_{1}, \delta_{2}$ & & \multicolumn{2}{|c|}{$0.30,0.46$} \\
\hline$D_{p, 1}, D_{p, 2}\left(\mathrm{~cm}^{2} / \mathrm{min}\right)$ & & \multicolumn{2}{|c|}{$2.9 \times 10^{-4}, 2.9 \times 10^{-4}$} \\
\hline$\gamma / \alpha$ & & \multicolumn{2}{|c|}{0.66} \\
\hline$\phi$ & & \multicolumn{2}{|l|}{1.74} \\
\hline Configuration & & \multicolumn{2}{|l|}{$2-2-2$} \\
\hline$L_{c}(\mathrm{~cm})$ & & \multicolumn{2}{|l|}{30} \\
\hline Diameter (cm) & & \multicolumn{2}{|l|}{0.75} \\
\hline$D V$ & & \multicolumn{2}{|l|}{$\sim 0.00$} \\
\hline$R_{p}(\mu \mathrm{m})$ & & \multicolumn{2}{|l|}{8.5} \\
\hline$C_{F, 1}, C_{F, 2}(\mathrm{~g} / \mathrm{L})$ & & \multicolumn{2}{|l|}{$1.9,1.7$} \\
\hline$Y_{1}, Y_{2}(\%)$ & $65.5,99.9$ & $99.9,99.9$ & $99.9,70.7$ \\
\hline$t_{s}(\min )$ & 20.5 & 21.5 & 22.5 \\
\hline \multicolumn{4}{|l|}{ Flowrates (mL/min) } \\
\hline Desorbent & & \multicolumn{2}{|l|}{0.5} \\
\hline Feed & & \multicolumn{2}{|l|}{0.045} \\
\hline Extract & & \multicolumn{2}{|l|}{0.15} \\
\hline Raffinate & & \multicolumn{2}{|l|}{0.395} \\
\hline$N_{D}^{*}$ & 4,447 & 4,664 & 4,881 \\
\hline$\underline{P}_{e b}{ }^{*}\left(L_{c} / 10 \varepsilon_{b} R_{p}\right)$ & & \multicolumn{2}{|c|}{$1,751(9,696)$} \\
\hline
\end{tabular}


Table B.2. Extended table of parameters for Case 2.

\begin{tabular}{|c|c|c|c|c|c|}
\hline & $2 A$ & 2B & $2 \mathrm{C}$ & 2D & $2 \mathbf{E}$ \\
\hline Authors & \multicolumn{5}{|c|}{ Houwing et al. } \\
\hline Year & & & \multicolumn{3}{|c|}{2003} \\
\hline Component 1 & & & \multicolumn{3}{|l|}{ BSA } \\
\hline Component 2 & & & \multicolumn{3}{|l|}{ Myoglobin } \\
\hline Design & & & \multicolumn{3}{|l|}{ Triangle } \\
\hline$\varepsilon_{p}$ & & & \multicolumn{3}{|l|}{0.98} \\
\hline$\varepsilon_{b}$ & & & \multicolumn{3}{|l|}{0.39} \\
\hline$K_{s e, 1}, K_{s e, 2}$ & & & \multicolumn{3}{|l|}{$0.65,0.88$} \\
\hline$\delta_{1}, \delta_{2}$ & & & \multicolumn{3}{|c|}{$0.73,0.95$} \\
\hline$D_{p, 1}, D_{p, 2}\left(\mathrm{~cm}^{2} / \mathrm{min}\right)$ & & & \multicolumn{3}{|c|}{$3.66 \times 10^{-5}, 7.2 \times 10^{-5}$} \\
\hline$\gamma / \alpha$ & & & \multicolumn{3}{|c|}{1.50} \\
\hline$\phi$ & & & \multicolumn{3}{|l|}{1.56} \\
\hline Configuration & & & \multicolumn{3}{|l|}{$2-2-2-2$} \\
\hline$L_{c}(\mathrm{~cm})$ & & & \multicolumn{3}{|l|}{8.9} \\
\hline Diameter $(\mathrm{cm})$ & & & \multicolumn{3}{|l|}{1.0} \\
\hline$D V$ & & & \multicolumn{3}{|l|}{0.06} \\
\hline$R_{p}(\mu \mathrm{m})$ & & & \multicolumn{3}{|l|}{100} \\
\hline$C_{F, 1}, C_{F, 2}(\mathrm{~g} / \mathrm{L})$ & & & \multicolumn{3}{|l|}{$1.9,1.7$} \\
\hline$Y_{1}, Y_{2}(\%)$ & $46.2,90.0$ & $60.5,90.0$ & $71.3,82.5$ & $86.7,60.0$ & $99.8,40.5$ \\
\hline$t_{s}(\min )$ & 1.14 & 1.14 & 1.14 & 1.14 & 1.14 \\
\hline \multicolumn{6}{|l|}{ Flowrates (mL/min) } \\
\hline Desorbent & 2.58 & 2.65 & 2.69 & 2.65 & 2.65 \\
\hline Feed & 0.15 & 0.15 & 0.15 & 0.15 & 0.15 \\
\hline Extract & 1.49 & 1.35 & 1.12 & 0.90 & 0.67 \\
\hline Raffinate & 1.23 & 1.46 & 1.72 & 1.91 & 2.13 \\
\hline$N_{D}^{*}$ & 0.11 & 0.11 & 0.11 & 0.11 & 0.11 \\
\hline$P_{e b}{ }^{*}\left(L_{c} / 10 \varepsilon_{b} R_{p}\right)$ & & & $38.0(228)$ & & \\
\hline
\end{tabular}


Table B.3. Extended table of parameters for Case 3.

\begin{tabular}{|c|c|}
\hline & Case $3^{*}$ \\
\hline Authors & Xie et al. \\
\hline Year & 2002 \\
\hline Component 1 & Insulin \\
\hline Component 2 & $\mathrm{ZnCl}_{2}$ \\
\hline Design & SWD \\
\hline$\varepsilon_{p}$ & 0.89 \\
\hline$\varepsilon_{b}$ & 0.35 \\
\hline$K_{s e, 1}, K_{s e, 2}$ & $0.74,0.99$ \\
\hline$\delta_{1}, \delta_{2}$ & $0.69,0.91$ \\
\hline$D_{p, 1}, D_{p, 2}\left(\mathrm{~cm}^{2} / \mathrm{min}\right)$ & $2.29 \times 10^{-5}, 1.65 \times 10^{-4}$ \\
\hline$\gamma / \alpha$ & 5.46 \\
\hline$\phi$ & 1.86 \\
\hline Configuration & $2-3-3-2$ \\
\hline$L_{c}(\mathrm{~cm})$ & 13.7 \\
\hline Diameter $(\mathrm{cm})$ & 5.1 \\
\hline$D V$ & 0.02 \\
\hline$R_{p}(\mu \mathrm{m})$ & 54 \\
\hline$C_{F, 1}, C_{F, 2}(\mathrm{~g} / \mathrm{L})$ & $69.5,0.303$ \\
\hline$Y_{1}, Y_{2}(\%)$ & $99.7,99.0$ \\
\hline$t_{s}(\min )$ & 33.7 \\
\hline \multicolumn{2}{|l|}{ Flowrates (mL/min) } \\
\hline Desorbent & 1.35 \\
\hline Feed & 1.10 \\
\hline Extract & 1.17 \\
\hline Raffinate & 1.29 \\
\hline$N_{D}^{*}$ & 8.9 \\
\hline$P_{e b}^{*}\left(L_{c} / 10 \varepsilon_{b} R_{p}\right)$ & $145(725)$ \\
\hline
\end{tabular}

